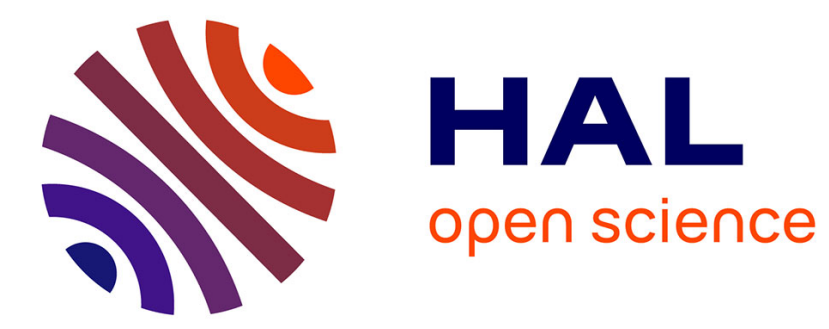

\title{
Convergence of nonlinear finite volume schemes for two-phase porous media flow on general meshes
}

Léo Agélas, Martin A. Schneider, Guillaume Enchéry, Bernd Flemisch

\section{To cite this version:}

Léo Agélas, Martin A. Schneider, Guillaume Enchéry, Bernd Flemisch. Convergence of nonlinear finite volume schemes for two-phase porous media flow on general meshes. 2020. hal-02903606

\section{HAL Id: hal-02903606 \\ https://hal.science/hal-02903606}

Preprint submitted on 21 Jul 2020

HAL is a multi-disciplinary open access archive for the deposit and dissemination of scientific research documents, whether they are published or not. The documents may come from teaching and research institutions in France or abroad, or from public or private research centers.
L'archive ouverte pluridisciplinaire HAL, est destinée au dépôt et à la diffusion de documents scientifiques de niveau recherche, publiés ou non, émanant des établissements d'enseignement et de recherche français ou étrangers, des laboratoires publics ou privés. 
Noname manuscript No.

(will be inserted by the editor)

\title{
Convergence of nonlinear finite volume schemes for two-phase porous media flow on general meshes
}

\author{
Léo Agélas • Martin Schneider • Guillaume Enchéry • \\ Bernd Flemisch
}

the date of receipt and acceptance should be inserted later

\begin{abstract}
In this work, we present an abstract finite volume discretization framework for incompressible immiscible two-phase flow through porous media. A-priori error estimates are derived that allow to prove the existence of discrete solutions and to establish the proof of convergence for schemes belonging to this framework. In contrast to existing publications, the proof is not restricted to a specific scheme and it does neither assume symmetry nor linearity of the flux approximations. Two nonlinear schemes, namely a nonlinear two-point flux approximation (NLTPFA) and a nonlinear multi-point flux approximation (NLMPFA) are presented and some properties of these schemes, e.g. saturation bounds, are proven. Furthermore, the numerical behavior of these schemes (e.g. accuracy, coercivity, efficiency or saturation bounds), is investigated for different test cases.
\end{abstract}

Keywords two-phase flow · porous medium - monotone schemes · finite volume methods . convergence analysis

\section{$1 \quad 1$ Introduction}

2 Flow through porous media occurs in a variety of technical engineering applications such as petroleum exploration and production, geological storage of carbon dioxide, hydrogeology, or geothermal energy. 4 Many challenging problems arise in the numerical simulation of complex fluid processes in reservoir simulation, subsurface contaminant transport and remediation, gas migration through engineered and geological barriers of deep radioactive waste repositories, sequestration of $\mathrm{CO}_{2}$, and other applications. The design of suitable discretization schemes for solving such applications is therefore essential. There is a large variety of discretization schemes that have been used for simulating multiphase flow in porous media, whereby mainly locally mass conservative schemes are used, which is

Léo Agélas

IFP Energies nouvelles, 1 \& 4 avenue du Bois-Préau, 92852 Rueil-Malmaison Cedex, France,

E-mail: leo.agelas@ifpen.fr

Martin Schneider

Institute for Modelling Hydraulic and Environmental Systems, University of Stuttgart, Pfaffenwaldring 61, 70569 Stuttgart, Germany,

E-mail: martin.schneider@iws.uni-stuttgart.de

Guillaume Enchéry

IFP Energies nouvelles, 1 \& 4 avenue du Bois-Préau, 92852 Rueil-Malmaison Cedex, France,

E-mail: guillaume.enchery@ifpen.fr

Bernd Flemisch

Institute for Modelling Hydraulic and Environmental Systems, University of Stuttgart, Pfaffenwaldring 61,70569 Stuttgart, Germany,

E-mail: bernd.flemisch@iws.uni-stuttgart.de 
essential when solving fluid dynamical processes. This is why finite volume schemes are the most commonly used methods for solving flow through porous media. A comparison and an overview of different schemes can be found in [14,33,38. For subsurface simulations, often corner-point grids are used to account for the different petrophysical properties that are associated to the control volumes (cells) of the grids. Solving partial differential equations on such corner-point grids with highly heterogeneous and anisotropic properties poses challenges on the discretization scheme. In our previous work, it has been demonstrated that so-called nonlinear finite volume schemes can be used for such grids and for complex applications [37,36, where the convergence of these nonlinear schemes has been proven for elliptic problems in 34. This work is an extension of 34 to incompressible immiscible two-phase porous media flow problems on general meshes. Besides a general discretization framework, including nonlinear flux discretization schemes, a-priori estimates are presented, which are used to show the existence of discrete solutions and to prove the convergence of schemes belonging to the presented discretization framework.

Previous publications include the convergence proof of a phase-based fully-upwind scheme with a two-point flux approximation, which was first analyzed for a one-dimensional setup in [7,32] and then extended in [24] to general higher dimensional grids. In this work, we establish the proof of convergence for the so-called fractional-flow formulation (global pressure-saturation formulation), which was theoretically analyzed (e.g. showing the existence of weak solutions) in [26, 10, 11. The fractional-flow approach treats the two-phase flow problem as a total fluid flow of a single mixed fluid, and then describes the individual phases as fractions of the total flow. This approach leads to two coupled equations: the global pressure equation and the saturation equation. For the mathematical analysis of different discretization schemes for this fractional-flow formulation we refer to [10, 20, 40 , 29,8 . The proof of convergence for schemes belonging to the gradient discretization framework (e.g 18) has been presented in 23. The gradient discretization method (GDM) is a recent framework for the numerical discretization and analysis of elliptic and parabolic PDEs. The usual GDM defines reconstruction operators (e.g. discrete gradient operators) on discrete solution spaces and discretizes the PDEs by replacing the continuous operators in the weak formulation by the corresponding discrete ones. The convergence of gradient schemes obtained in [7,23] is in fact based upon a weakstar convergence (weak-strong convergence) argument which states that if $f_{n} \rightarrow f$ is weak-star convergent in the dual space $X^{*}$ of a Banach space $X$ and $x_{n} \rightarrow x$ converges strongly in $X$, then $\left[f_{n}, x_{n}\right] \rightarrow[f, x]$ as $n \rightarrow \infty$. The use of this argument in the case of gradient schemes is possible because only one discrete gradient reconstruction operator $\nabla_{\mathcal{D}}$ is used in the discrete problem, which allows to get both weak and strong convergence in the duality bracket thanks to the limit-conformity and consistency properties required for these methods. Thus, by using an argument of weak-strong convergence, the proof of convergence follows by establishing some compactness results (see Section 3.3 of [8] and Theorem 3.7 in [23]).

However, despite its flexibility, the usual GDM does not seem to cover some important families of numerical methods, in particular some finite volume schemes such as the two-point flux approximation (TPFA), the multi-point flux approximation MPFA-L/G schemes, MPFA-O schemes on general meshes except some particular meshes for which they become symmetric (simplex, parallelogram), or nonlinear schemes. These non-symmetric schemes do not belong to the family of gradient schemes, because two different gradient reconstruction operators $\nabla_{\mathcal{D}}$ and $\widetilde{\nabla}_{\mathcal{D}}$ are needed in the discrete formulation, where one of the operators is strong (in the sense of the consistency) and the other one is weak (in the sense of the limit-conformity). Due to these two different gradient reconstruction operators, which appear in the duality bracket terms of the weak formulation, the weak-strong convergence argument cannot be used to get the proof of convergence. This is the main reason why the proof of convergence for non-symmetric schemes is quite different from the one used for schemes encompassed by the gradient discretisation framework. The proof of convergence for non-symmetric schemes requires to establish a-priori error estimates (see the proof of Theorem 1 in [1], Lemma 5.7 and Theorem 5.1 in [2, Theorem 1 in 34 and the asymmetric gradient discretization framework in [16]) depending on duality bracket terms, which involve two discrete gradient reconstruction operators and thus allow the use of weak-strong convergence and compactness arguments. This is done in this article, where we give, after establishing a-priori error estimates, the proof of convergence for the two-phase flow problem of cell-centered finite volume schemes which are possibly unsymmetric 
and nonlinear. The proof is based on a-priori error estimates combined with compactness arguments, where our assumptions are compatible with field applications (discontinuous data, fully nonlinear models, etc.). These are, at least to our knowledge, novel results for general parabolic PDEs and differ from recent proofs which are essentially based on weak-strong convergence arguments (see [8, 23]). Thus our proof appears to be technically quite difficult because of the a-priori error estimates that have to be additionally established.

Furthermore, most of the existing literature either neglect capillary pressure or buoyancy terms in their mathematical analysis and only consider linear flux approximations. This is not done in this work, where all terms are considered and the fluxes are allowed to be nonlinear. Such nonlinear flux approximations have the advantage that they are consistent and satisfy saturation bounds.

This work is organized as follows: In Section 2, the mathematical formulation of the two-phase flow problem using the fractional-flow formulation is presented. In Sections 3 and 4 a general finite volume discretization framework is introduced and the proof of convergence is given. This general framework also includes nonlinear schemes. Two representatives of such schemes are presented in Section 5 , where also some fundamental properties of these schemes are proven. Finally, these schemes are numerically investigated in Section 6 for a quasi one-dimensional setup and a two-dimensional test case including gravity and capillary pressure effects.

\section{Mathematical formulation of a two-phase flow problem}

\subsection{Continuous form}

Let $\Omega \subset \mathbb{R}^{d}, d \in \mathbb{N}^{*}$, be an open bounded connected polygonal domain with boundary $\partial \Omega$ and $d$-dimensional measure $|\Omega|$. On $\Omega$ and for all $t \in(0, T)(T>0)$, we define the following two-phase porous-media flow problem, where the phases are assumed to be incompressible and immiscible with a rigid porous matrix,

$$
\begin{aligned}
\phi u_{t}-\nabla \cdot\left(\lambda_{1}(u) \boldsymbol{\Lambda}\left(\nabla p_{1}-\varrho_{1} \mathbf{g}\right)\right) & =f_{1}(c) s^{+}-f_{1}(u) s^{-}, \\
\phi(1-u)_{t}-\nabla \cdot\left(\lambda_{2}(u) \boldsymbol{\Lambda}\left(\nabla p_{2}-\varrho_{2} \mathbf{g}\right)\right) & =f_{2}(c) s^{+}-f_{2}(u) s^{-} .
\end{aligned}
$$

Here, $u$ denotes the saturation of the wetting phase; $p_{1}, p_{2}$ the wetting and non-wetting pressures linked together through the capillary pressure $p_{c}=p_{2}-p_{1} ; \phi$ the porosity; $\boldsymbol{\Lambda}$ a symmetric permeability tensor; $\varrho_{1}, \varrho_{2}$ the phase densities; $\mathbf{g}=(0,0,-g)^{T}$ the gravity vector $(g>0) ; s^{+}, s^{-}$the source and sink terms; $c$ the inflow wetting saturation; $\lambda_{1}$ and $\lambda_{2}$ the wetting and non-wetting phase mobilities; and $f_{1}, f_{2}$ the fractional-flow functions, which are given as

$$
f_{1}=\frac{\lambda_{1}}{\lambda_{\mathrm{T}}}, \quad f_{2}=\frac{\lambda_{2}}{\lambda_{\mathrm{T}}}
$$

where $\lambda_{\mathrm{T}}=\lambda_{1}+\lambda_{2}$ is the total mobility.

Using these quantities, problem (1) can be rewritten in the fractional-flow form

$$
\begin{aligned}
\phi u_{t}+\nabla \cdot\left(f_{1} \mathbf{v}_{\mathrm{T}}-\boldsymbol{\Lambda} \nabla \psi(u)+\left(\varrho_{1}-\varrho_{2}\right) f_{1} \lambda_{2} \boldsymbol{\Lambda} \mathbf{g}\right) & =f_{1}(c) s^{+}-f_{1}(u) s^{-} \\
\nabla \cdot \mathbf{v}_{\mathrm{T}} & =s^{+}-s^{-}
\end{aligned}
$$

where we have introduced the total velocity

$$
\mathbf{v}_{\mathrm{T}}=-\lambda_{\mathrm{T}}(u) \boldsymbol{\Lambda}\left(\nabla p-\varrho_{f} \mathbf{g}\right),
$$

with the average fluid density

$$
\varrho_{f}=\varrho_{1} f_{1}+\varrho_{2} f_{2}
$$

the global pressure

$$
p=p_{1}-\int_{u}^{1} f_{2}(v) p_{c}^{\prime}(v) \mathrm{d} v,
$$


96

and the following function

$$
\psi(u)=-\int_{0}^{u} \lambda_{1}(v) f_{2}(v) p_{c}^{\prime}(v) \mathrm{d} v .
$$

Initial conditions for Problem (3) are given for the wetting saturation

$$
u(., 0)=u_{\text {init }} \text { in } \Omega .
$$

Additionally, for simplicity, we assume homogeneous zero Dirichlet boundary conditions

$$
u(\mathbf{x}, t)=0, \quad p(\mathbf{x}, t)=0, \quad \text { on } \partial \Omega \times(0, T) .
$$

In the following, we consider problem (3) with the two unknowns $(u, p)$ and make the following assumptions. For simplicity we do not introduce residual saturations such that the effective saturation corresponds to $u$.

Hypotheses 1 We assume that:

${ }_{103}(A 1) \phi \in L^{\infty}(\Omega)$ with $\phi \in[\phi, \bar{\phi}]$ almost everywhere (a.e.) in $\Omega$ (without loss of generality, we assume $\phi=1$ $104 \quad$ in the mathematical analysis of the finite volume scheme),

${ }_{105}\left(\right.$ A2) $\boldsymbol{\Lambda}$ is symmetric and there exist $0<\alpha_{0}<\beta_{0}<+\infty$ so that the spectrum of $\boldsymbol{\Lambda}$ is contained in $\left[\alpha_{0}, \beta_{0}\right]$ $106 \quad$ a.e. in $\Omega$,

107 (A3) $u_{\text {init }} \in L^{\infty}(\Omega)$, with $u_{\text {init }} \in[0,1]$ a.e.,

108 (A4) $c \in L^{\infty}(\Omega \times(0, T))$, with $c \in[0,1]$ a.e.,

109 (A5) $s^{+}, s^{-} \in L^{2}(\Omega \times(0, T)), s^{+} \geq 0$ and $s^{-} \geq 0$ a.e.,

${ }_{110}(A 6) \lambda_{1}: \mathbb{R} \mapsto\left[0, \bar{\lambda}_{1}\right]$ is a nondecreasing Lipschitz continuous function such that (s.t.)

$$
\lambda_{1}(x)=0, \quad \forall x \in(-\infty, 0], \quad \lambda_{1}(x)=\bar{\lambda}_{1}>0, \quad \forall x \in[1, \infty),
$$

${ }_{111}\left(\right.$ A7) $\quad \lambda_{2}: \mathbb{R} \mapsto\left[0, \bar{\lambda}_{2}\right]$ is a nonincreasing Lipschitz continuous function s.t.

$$
\lambda_{2}(x)=\bar{\lambda}_{2}>0, \quad \forall x \in(-\infty, 0], \quad \lambda_{2}(x)=0, \quad \forall x \in[1, \infty),
$$

${ }_{112}(A 8) \psi \in C([0,1])$ with $\psi(0)=0$, is a strictly increasing Lipschitz-continuous function. The function $\psi$ is 113

$$
\psi(u)= \begin{cases}\Xi \psi(1)(u-1)+\psi(1) & \text { if } u>1 \\ \Xi u & \text { if } u<0\end{cases}
$$

with $\Xi>0$. We denote by $L_{\psi}$ the Lipschitz constant of $\psi$ over $\mathbb{R}$. At last, there exist $C_{1, \psi} \geq 0$, $C_{2, \psi} \geq 0$ so that, for all $u \in \mathbb{R}$,

$$
|\psi(u)| \geq C_{1, \psi}|u|-C_{2, \psi}
$$

Using the assumptions (A6) and (A7) we set $\underline{\lambda}=\min _{x \in \mathbb{R}} \lambda_{\mathrm{T}}(x)$ and $\bar{\lambda}=\max _{x \in \mathbb{R}} \lambda_{\mathrm{T}}(x)$. We also introduce the function

$$
\Psi(s)=\int_{0}^{s} \psi(x) \mathrm{d} x, \quad \forall s \in \mathbb{R} .
$$

Thanks to the Lipschitz continuity of $\psi$, the fact that $\psi$ is nondecreasing and that $\psi(0)=0$, the function $\Psi$ satisfies the following inequality (see proof of Lemma 13 in section Appendix):

$$
0 \leq \frac{\psi(s)^{2}}{2 L_{\psi}} \leq \Psi(s)=\int_{0}^{s}(\psi(x)-\psi(0)) \mathrm{d} x \leq L_{\psi} \frac{s^{2}}{2}
$$

Furthermore, under assumption (A8) we deduce that $2\left(\psi(s)^{2}+C_{2, \psi}^{2}\right) \geq C_{1, \psi}^{2}|s|^{2}$. Hence, using (12), we obtain

$$
\Psi(s) \geq \frac{C_{1, \psi}^{2}|s|^{2}-2 C_{2, \psi}^{2}}{4 L_{\psi}} .
$$

The monotonicity of $\psi$ implies that $\Psi$ is a convex function such that for all $s_{1}, s_{2} \in \mathbb{R}$

$$
\Psi\left(s_{2}\right)-\Psi\left(s_{1}\right) \leq \psi\left(s_{2}\right)\left(s_{2}-s_{1}\right) .
$$




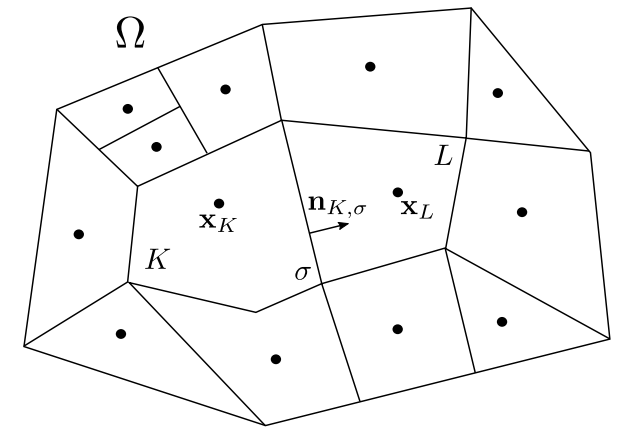

Fig. 1 An example of admissible mesh for $d=2$.

\subsection{Weak form}

Under Hypotheses $1 .(\bar{p}, \bar{u})$ is a weak solution of $(3)$ if

$-\bar{p} \in L^{2}\left(0, T ; H_{0}^{1}(\Omega)\right)$,

$-\bar{u} \in L^{2}(\Omega \times(0, T))$

$-\psi(\bar{u}) \in L^{2}\left(0, T ; H_{0}^{1}(\Omega)\right)$,

and if, for all $\varphi \in L^{2}\left(0, T ; H_{0}^{1}(\Omega)\right)$ s.t. $\varphi_{t} \in L^{2}(\Omega \times(0, T))$ and $\varphi(., T)=0$ a.e., we have

$$
\begin{aligned}
\int_{0}^{T} \int_{\Omega}\left[-\phi \bar{u} \varphi_{t}-\left(f_{1} \mathbf{v}_{\mathrm{T}}-\boldsymbol{\Lambda}\right.\right. & \left.\left.\left.\nabla \psi(\bar{u})+\left(\varrho_{1}-\varrho_{2}\right) f_{1} \lambda_{2} \mathbf{\Lambda} \mathbf{g}\right)\right) \nabla \varphi\right] \mathrm{d} x \mathrm{~d} t \\
& =\int_{\Omega} \phi u_{\text {init }}(\mathbf{x}) \varphi(\mathbf{x}, 0) \mathrm{d} x+\int_{0}^{T} \int_{\Omega}\left(f_{1}(c) s^{+}-f_{1}(\bar{u}) s^{-}\right) \varphi \mathrm{d} x \mathrm{~d} t \\
-\int_{0}^{T} \int_{\Omega} \mathbf{v}_{\mathrm{T}} \cdot \nabla \varphi \mathrm{d} x \mathrm{~d} t & =\int_{0}^{T} \int_{\Omega}\left(s^{+}-s^{-}\right) \varphi \mathrm{d} x \mathrm{~d} t
\end{aligned}
$$

\section{Finite volume discretization}

Before giving a finite volume discretization of (15), we introduce a few notations and definitions.

\subsection{Discretization of the space and time domains and their regularity}

We first define the spatial discretization which includes general polygonal meshes (see Figure 1).

Definition 1 (Spatial discretization) A spatial discretization $\mathcal{D}$ is a triplet $\mathcal{D}=(\mathcal{T}, \mathcal{E}, \mathcal{P})$, where

(i) $\mathcal{T}$ (the cells or control volumes) is a finite family of non-empty connected open disjoint subsets of $\Omega$ s.t. $\bar{\Omega}=\cup_{K \in \mathcal{T}} \bar{K}$. For all cells $K \in \mathcal{T},|K|>0$ denotes its $d$-dimensional measure (the volume) and $\partial K \stackrel{\text { def }}{=} \bar{K} \backslash K$ its boundary. The size of the discretization is defined by $h_{\mathcal{D}} \stackrel{\text { def }}{=} \sup _{K \in \mathcal{T}} \operatorname{diam}(K)$. The number of cells is indicated by $n_{\mathcal{T}}$.

(ii) $\mathcal{E}$ (the faces) is a finite family of subsets of $\bar{\Omega}$ s.t., for all $\sigma \in \mathcal{E}, \sigma$ is a non-empty closed subset of a hyperplane of $\mathbb{R}^{d}$ with $(d-1)$-dimensional measure $|\sigma|>0$ (the area), and the intersection of two different faces has zero $(d-1)$-dimensional measure. For all $K \in \mathcal{T}$, we assume that there exists a subset $\mathcal{E}_{K}$ of $\mathcal{E}$ s.t. $\partial K=\cup_{\sigma \in \mathcal{E}_{K}} \sigma$. For any $\sigma \in \mathcal{E}$, either $\mathcal{T}_{\sigma} \stackrel{\text { def }}{=}\left\{K \in \mathcal{T} \mid \sigma \in \mathcal{E}_{K}\right\}$ has exactly one element (if $\sigma \subset \partial \Omega$ ) or $\mathcal{T}_{\sigma}$ has exactly two elements (inner face); the sets of inner and boundary faces are denoted by $\mathcal{E}_{\text {int }}$ and $\mathcal{E}_{\text {ext }}$, respectively. The face evaluation points (interpolation points) are denoted by $\mathbf{x}_{\sigma}$ (not required to be the barycenters). For all $K \in \mathcal{T}$ and $\sigma \in \mathcal{E}_{K}$, we denote by $\mathbf{n}_{K, \sigma}$ the unit vector that is normal to $\sigma$ and outward to $K$. 
(iii) $\mathcal{P}=\left\{\mathbf{x}_{K}\right\}_{K \in \mathcal{T}}$ (the cell centers, not required to be the barycenters) is a family of points of $\Omega$ s.t. $\mathbf{x}_{K} \in K$. We assume that there is $\alpha>0$ such that for all $K \in \mathcal{T}, K$ is star-shaped with respect to all the points in a ball of radius $\alpha \operatorname{diam}(K)$ and, in particular, to $\mathbf{x}_{K}$. For all $K \in \mathcal{T}$ and for all $\sigma \in \mathcal{E}_{K}, d_{K, \sigma}$ denotes the Euclidean distance between $\mathbf{x}_{K}$ and the hyperplane including $\sigma$, and $\Delta_{K, \sigma}$ denotes the convex hull of $\mathbf{x}_{K}$ and $\sigma$. In addition, we denote, for all $\sigma \in \mathcal{E}$, by $\Delta_{\sigma}=$ Interior $\left(\bigcup_{K \in \mathcal{T}_{\sigma}} \overline{\Delta_{K, \sigma}}\right)$ and by $\Delta=\left\{\Delta_{\sigma}, \sigma \in \mathcal{E}\right\}$.

Let us remark with the notations of Definition 3.1. that, since $\frac{|\sigma| d_{K, \sigma}}{d}$ is the measure of the convex hull $\Delta_{K, \sigma}$ of $\mathbf{x}_{K}$ and $\sigma$, we have

$$
\forall K \in \mathcal{T}, \quad \sum_{\sigma \in \mathcal{E}_{K}}|\sigma| d_{K, \sigma}=d|K|
$$

In the following of this work, a few regularity assumptions are made on the spatial discretization for the convergence analysis of the scheme. Therefore, we now introduce the notion of an admissible spatial discretization.

Definition 2 (Admissible spatial discretization) Let $\mathcal{D}$ be a spatial discretization of $\Omega$ in the sense of Definition 1 . This discretization is admissible if there exist $0<\zeta_{1}, \zeta_{2}, \zeta_{3}, \zeta_{4}, \zeta_{5}<+\infty$ s.t.

$$
\left|\mathcal{E}_{K}\right| \leq \zeta_{1}, \quad \min _{K \in \mathcal{T}, \sigma \in \mathcal{E}_{K}} \frac{|\sigma|}{\operatorname{diam}(K)^{d-1}} \geq \zeta_{2},
$$

$$
\min _{K \in \mathcal{T}, \sigma \in \mathcal{E}_{K}} \frac{d_{K, \sigma}}{\operatorname{diam}(K)} \geq \zeta_{3}, \min _{\sigma \in \mathcal{E}_{\text {int }}, \mathcal{T}_{\sigma}=\{K, L\}} \frac{\min \left(d_{K, \sigma}, d_{L, \sigma}\right)}{\max \left(d_{K, \sigma}, d_{L, \sigma}\right)} \geq \zeta_{4}, \min _{K \in \mathcal{T}} \frac{\operatorname{diam}(K)}{h_{\mathcal{D}}} \geq \zeta_{5}
$$

The next two definitions allow us to precise the concept of admissible discretization for the whole space-time domain and to introduce the notion of admissible family for these discretizations that will be used for the convergence study. For this, we use the definition $\llbracket 0, N \rrbracket \stackrel{\text { def }}{=}\{0, \cdots, N\}$.

Definition 3 (Admissible space-time discretization) The pair $\mathscr{D}=\left(\mathcal{D}, \mathcal{D}^{t}\right)$ is a space-time discretization of $\Omega \times(0, T)$ if:

$-\mathcal{D}$ is a spatial discretization in the sense of Definition 1

$-\mathcal{D}^{t}=\bigcup_{n \in \llbracket 0, N \rrbracket} I_{n}$ with $I_{n}=\left[t^{(n)}, t^{(n+1)}\left[,\left\{t^{(n)}\right\}_{n=0, \cdots, N}\right.\right.$ such that $t^{(0)}=0, t^{(N+1)}=T$, and $\delta t^{\left(n+\frac{1}{2}\right)}=t^{(n+1)}-t^{(n)}>0$, for all $n \in \llbracket 0, N \rrbracket$.

The maximum time step size of a space-time discretization is denoted by

$$
|\delta t|=\max _{n=0, \cdots, N} \delta t^{\left(n+\frac{1}{2}\right)} .
$$

\section{It is said to be admissible if $\mathcal{D}$ is admissible according to Definition 2}

Definition 4 (Admissible family of space-time discretizations) A family of space-time discretizations $\left\{\mathscr{D}_{m}\right\}_{m \in \mathbb{N}}$ is admissible if $h_{\mathcal{D}_{m}} \rightarrow 0,\left|\delta t_{m}\right| \rightarrow 0$ as $m \rightarrow \infty$, and for each $m, \mathscr{D}_{m}$ is admissible in the sense of Definition 3 where the parameters $\zeta_{1}, \zeta_{2}, \zeta_{3}, \zeta_{4}, \zeta_{5}$ do not depend on $m$.

In what follows, when referring to a generic element $\mathscr{D}_{m}$ of an admissible family of discretizations $\left\{\mathscr{D}_{m}\right\}_{m \in \mathbb{N}}$, the subscript $m$ will be dropped for the ease of reading in cases where no ambiguity arises.

\subsection{Further notations and discrete tools}

In this section, we introduce further notations and some discrete tools which are needed for the analysis of the discrete scheme. 


\subsubsection{Notations}

In the sequel, we use the following notations

- for any $V \subset \Omega$ and $\Phi \in L^{1}(V),\langle\Phi\rangle_{V} \stackrel{\text { def }}{=}|V|^{-1} \int_{V} \Phi \mathrm{d} x$ which is meant component-wise for functions with vector or tensor values,

$-\mathcal{L}(E ; F)$ represents the vector space of bounded linear operators from $E$ to $F$.

\subsubsection{Discrete spaces}

First, we define discrete spaces on $\Omega$. The space of piecewise constant functions on $\mathcal{Q} \in\{\mathcal{T}, \Delta\}$ is defined as

$$
H_{\mathcal{Q}}(\Omega) \stackrel{\text { def }}{=}\left\{v \in L^{2}(\Omega) \mid v_{\mathscr{K}} \stackrel{\text { def }}{=} v_{\mid \mathscr{K}} \in \mathbb{P}^{0}(\mathscr{K}), \forall \mathscr{K} \in \mathcal{Q}\right\} .
$$

With this, for any $v \in L^{2}(\Omega)$, we denote by $v_{\mathcal{Q}}$ the element of $H_{\mathcal{Q}}(\Omega)$ such that for all $\mathscr{K} \in \mathcal{Q}$, $\left(v_{\mathcal{Q}}\right)_{\mathscr{K}}=\langle v\rangle_{\mathscr{K}}$. For $v \in H_{\Delta}(\Omega)$ we often use the abbreviation $v_{\sigma}$ instead of $v_{\Delta_{\sigma}}$.

Next, we define discrete spaces on $\Omega \times(0, T)$. Here, the space of piecewise constant functions on $\mathcal{Q} \in\{\mathscr{T}, \mathscr{E}\}$, with $\mathscr{T} \stackrel{\text { def }}{=} \mathcal{T} \times \mathcal{D}^{t}$ and $\mathscr{E} \stackrel{\text { def }}{=} \Delta \times \mathcal{D}^{t}$, is given as

$$
H_{\mathcal{Q}}(\Omega \times(0, T)) \stackrel{\text { def }}{=}\left\{v \in L^{2}(\Omega \times(0, T)) \mid v_{\mathscr{K}}^{(n)} \stackrel{\text { def }}{=} v_{\mid \mathscr{K} \times I_{n}} \in \mathbb{P}^{0}\left(\mathscr{K} \times I_{n}\right), \forall\left(\mathscr{K}, I_{n}\right) \in \mathcal{Q}\right\} .
$$

In the same way, for any $v \in L^{2}(\Omega \times(0, T))$, we denote by $v_{\mathcal{Q}}$ the element of $H_{\mathcal{Q}}(\Omega \times(0, T))$ such that for all $\left(\mathscr{K}, I_{n}\right) \in \mathcal{Q},\left(v_{\mathcal{Q}}\right)_{\mathscr{K}}^{(n)}=\langle v\rangle_{\mathscr{K} \times I_{n}}$. With this, for each $v \in H_{\mathcal{Q}}(\Omega \times(0, T))\left(\mathcal{Q}=\tilde{\mathcal{Q}} \times \mathcal{D}^{t}\right.$ and $\tilde{\mathcal{Q}} \in\{\mathcal{T}, \Delta\})$ and for each $t \in I_{n}$, we define $v^{(n)}=v(t) \in H_{\tilde{\mathcal{Q}}}(\Omega)$ s.t. $(v(t))_{\mathscr{K}}=\langle v(\cdot, t)\rangle_{\mathscr{K}}$ for all $\mathscr{K} \in \tilde{\mathcal{Q}}$.

\subsubsection{Discrete operators and norms}

We now introduce a general trace reconstruction operator, which allows to define discrete gradients and $H^{1}$-norms on the spaces $H_{\mathcal{Q}}$.

Definition 5 (Trace reconstruction operator) A trace reconstruction operator is a set of bounded linear operators $I$, such that $I=\left\{I_{\sigma}\right\}_{\sigma \in \mathcal{E}}, I_{\sigma} \in \mathcal{L}\left(H_{\mathcal{T}}(\Omega) ; \mathbb{P}^{0}(\sigma)\right)$, and $I_{\sigma} v=0$ for all $v \in H_{\mathcal{T}}$ and $\sigma \in \mathcal{E}_{\text {ext }}$.

Among these operators, we will consider the ones, denoted by $\Upsilon \stackrel{\text { def }}{=}\left\{\Upsilon_{\sigma}\right\}_{\sigma \in \mathcal{E}}$, for which there exist, for all $\sigma \in \mathcal{E}_{\text {int }}$ with $\mathcal{T}_{\sigma}=\{K, L\}$, two non-negative values, $\theta_{K, \sigma}$ and $\theta_{L, \sigma}$, such that $\theta_{K, \sigma}+\theta_{L, \sigma}=1$ and which are given by

$$
\Upsilon_{\sigma} v= \begin{cases}\theta_{L, \sigma} v_{K}+\theta_{K, \sigma} v_{L} & \text { if } \sigma \in \mathcal{E}_{\text {int }} \\ 0 & \text { if } \sigma \in \mathcal{E}_{\text {ext }}\end{cases}
$$

We denote by $\mathscr{R}_{\mathcal{E}}$ the set of operators satisfying $(20)$. Of special interest is the trace reconstruction operator $\gamma=\left\{\gamma_{\sigma}\right\}_{\sigma \in \mathcal{E}}$ that is defined, for all $v \in H_{\mathcal{T}}(\Omega)$, by

$$
\gamma_{\sigma} v= \begin{cases}\frac{d_{L, \sigma} v_{K}+d_{K, \sigma} v_{L}}{d_{K, \sigma}+d_{L, \sigma}} & \text { if } \sigma \in \mathcal{E}_{\text {int }}, \\ 0 & \text { if } \sigma \in \mathcal{E}_{\text {ext }} .\end{cases}
$$

Then, for any trace reconstruction operator $I=\left\{I_{\sigma}\right\}_{\sigma \in \mathcal{E}}$ matching Definition 5 and for any $v \in$ $H_{\mathcal{T}}(\Omega)$, we define

- a discrete gradient with values in $\left(H_{\mathcal{T}}(\Omega)\right)^{d}$ :

$$
\left(\widetilde{\nabla}_{\mathcal{D}, I} v\right)_{K} \stackrel{\text { def }}{=} \frac{1}{|K|} \sum_{\sigma \in \mathcal{E}_{K}}|\sigma|\left(I_{\sigma} v-v_{K}\right) \mathbf{n}_{K, \sigma},
$$


- a discrete norm:

$$
\|v\|_{\mathcal{T}, I} \stackrel{\text { def }}{=}\left(\sum_{K \in \mathcal{T}} \sum_{\sigma \in \mathcal{E}_{K}} \frac{|\sigma|}{d_{K, \sigma}}\left|I_{\sigma} v-v_{K}\right|^{2}\right)^{\frac{1}{2}}
$$

where for $I=\gamma$ we use the simplified notation $\|v\|_{\mathcal{T}} \stackrel{\text { def }}{=}\|v\|_{\mathcal{T}, \gamma}$,

- a discrete dual semi-norm for all $u \in L^{2}(\Omega)$ :

$$
\|u\|_{-1, \mathcal{T}} \stackrel{\text { def }}{=} \sup \left\{\int_{\Omega} u(x) w(x) d x: w \in H_{\mathcal{T}}(\Omega),\|w\|_{\mathcal{T}}=1\right\}
$$

- the extensions of both previous norms to the space $H_{\mathscr{T}}(\Omega \times(0, T))$ :

$$
\|v\|_{\mathscr{T}} \stackrel{\text { def }}{=}\left(\int_{0}^{T}\|v(t)\|_{\mathcal{T}}^{2} \mathrm{~d} t\right)^{1 / 2} \text { and }\|v\|_{-1, \mathscr{T}} \stackrel{\text { def }}{=}\left(\int_{0}^{T}\|v(t)\|_{-1, \mathcal{T}}^{2} \mathrm{~d} t\right)^{1 / 2} .
$$

Finally, for all $v \in H_{\mathscr{T}}(\Omega \times(0, T))$ and $n=0, \cdots, N-1$, we also define the discrete time derivative of $v$,

$$
\left(\delta_{t} v\right)_{K}^{(n)} \stackrel{\text { def }}{=} \frac{v_{K}^{(n+1)}-v_{K}^{(n)}}{\delta t^{\left(n+\frac{1}{2}\right)}}
$$

and, for all $K \in \mathcal{T}$ and $\sigma \in \mathcal{E}_{K}$, we denote by

$$
F_{K, \sigma}: H_{\mathcal{T}}(\Omega) \times H_{\mathcal{T}}(\Omega) \mapsto \mathbb{P}^{0}(\sigma)
$$

a numerical flux designed to approximate the flow induced by the normal component of a gradient term with respect to $\mathbf{n}_{K, \sigma}$. In this work, we assume that the fluxes are locally mass conservative, meaning that for any $\sigma \in \mathcal{E}_{\text {int }}$ with $\mathcal{T}_{\sigma}=\{K, L\}$

$$
F_{K, \sigma}(u, v)+F_{L, \sigma}(u, v)=0 .
$$

\subsection{Definition of the scheme}

Using the notations introduced in Sections 3.1 3.2, a finite volume discretization of problem (15), along with an implicit Euler scheme for the time discretization, consists in computing a pair $(u, p) \in$ $\left[H_{\mathscr{T}}(\Omega \times(0, T))\right]^{2}$ s.t., for all $n \in\{0, \cdots, N-1\}$ and $K \in \mathcal{T}$ :

$$
\begin{aligned}
|K| \frac{u_{K}^{(n+1)}-u_{K}^{(n)}}{\delta t^{\left(n+\frac{1}{2}\right)}} & +\sum_{\sigma \in \mathcal{E}_{K}} f_{1}\left(u_{1, \sigma}^{(n+1)}\right) \mathbf{v}_{K, \sigma}^{(n+1)}+f_{1}\left(u_{2, \sigma}^{(n+1)}\right) \lambda_{2}\left(u_{3, \sigma}^{(n+1)}\right)\left(\varrho_{1}-\varrho_{2}\right) G_{K, \sigma} \\
& -\sum_{\sigma \in \mathcal{E}_{K}} F_{K, \sigma}\left(\psi\left(u^{(n+1)}\right), \psi\left(u^{(n+1)}\right)\right)=|K|\left(f_{1}\left(c_{K}\right) s_{K}^{+}-f_{1}\left(u_{K}^{(n+1)}\right) s_{K}^{-}\right),
\end{aligned}
$$

$$
\sum_{\sigma \in \mathcal{E}_{K}} \mathbf{v}_{K, \sigma}^{(n+1)}=|K|\left(s_{K}^{+}-s_{K}^{-}\right)
$$

Note that 28b also holds for $n=-1$. In the previous discrete system (28), we have used the following notations

$$
\mathbf{v}_{K, \sigma}^{(n+1)}=-\lambda_{\mathrm{T}}\left(u_{1, \sigma}^{(n+1)}\right)\left(F_{K, \sigma}\left(p^{(n+1)}, p^{(n+1)}\right)-\varrho_{f}\left(u_{0, \sigma}^{(n+1)}\right) G_{K, \sigma}\right),
$$

${ }_{225}$ with $u_{0, \sigma}^{(n+1)}=0.5\left(u_{K}^{(n+1)}+u_{L}^{(n+1)}\right)$,

$$
G_{K, \sigma}=|\sigma|\left(\delta_{K, \sigma} \Lambda_{K} \mathbf{n}_{K, \sigma} \cdot \mathbf{g}-\delta_{L, \sigma} \Lambda_{L} \mathbf{n}_{L, \sigma} \cdot \mathbf{g}\right),
$$


241 (P2) for any bounded function $\chi$ and $\alpha_{m} \in H_{\Delta_{m}}(\Omega)$, a $\mathcal{T}_{m}, \chi, \alpha_{m}$ is continuous, i.e., there is $0<C_{\chi}<+\infty$ 242

$$
\left|a_{\mathcal{T}_{m}, \chi, \alpha_{m}}(u, v, w)\right| \leq C_{\chi}\|v\|_{\mathcal{T}_{m}}\|w\|_{\mathcal{T}_{m}}
$$

${ }_{243}$ (P3) the finite volume scheme is coercive, i.e., there is $0<\hat{C}_{1}<+\infty$ independent of $m$ s.t. for $\chi=\lambda \lambda_{\mathrm{T}}$ and 244 $\chi=1$, for all $(v, w) \in\left[H_{\mathcal{T}_{m}}(\Omega)\right]^{2}$ and for any $\alpha_{m} \in H_{\Delta_{m}}(\Omega)$

$$
a_{\mathcal{T}_{m}, \chi, \alpha}(v, w, w) \geq \hat{C}_{1}\|w\|_{\mathcal{T}_{m}}^{2}
$$

245 (P4) For $\chi=1, \chi=\lambda_{\mathrm{T}}$ or $\chi=\lambda_{1}, a_{\mathcal{T}_{m}, \chi}$. is weakly consistent on $L^{2}(0, T ; \mathfrak{D})$, i.e., for all $\varphi \in L^{2}(0, T ; \mathfrak{D})$, $\epsilon_{\mathscr{D}_{m}}(\varphi) \rightarrow 0$ as $m \rightarrow \infty$, where

$$
\epsilon_{\mathscr{D}_{m}}(\varphi) \stackrel{\text { def }}{=} \max _{(u, v, w) \in \mathscr{P}^{m}} \inf _{\Upsilon_{m} \in \mathscr{R}_{\mathcal{E}_{m}}} \frac{1}{\|w\| \mathscr{T}_{m}}\left|\int_{0}^{T} a_{\mathcal{T}_{m}, \chi, v(t)}\left(u(t), \varphi_{\mathscr{T}_{m}}(t), w(t)\right) d t-\int_{0}^{T} \int_{\Omega} \chi(v) \Lambda \nabla \varphi \cdot \widetilde{\nabla}_{\mathcal{D}_{m}, \Upsilon_{m}} w d x d t\right|
$$

$$
\text { where } \mathscr{P}^{m} \stackrel{\text { def }}{=}\left\{(u, v, w) \mid(u, w) \in\left[H_{\mathscr{T}_{m}}(\Omega \times(0, T))\right]^{2}, w \neq 0, v \in H_{\mathscr{E}_{m}}(\Omega \times(0, T))\right\} \text {. }
$$

By using the fact that $\mathfrak{D}$ is a dense subspace of $H_{0}^{1}(\Omega)$, we extend in Proposition 4 property $(\mathrm{P} 4)$ to the space $L^{2}\left(0, T ; H_{0}^{1}(\Omega)\right)$. This result is stated and proved in Section 8.1

\subsection{A priori estimates}

In this section, we establish several estimates that will be used in Sections 4.2 and 4.3 to prove the existence of discrete solutions and the convergence of the scheme. 
Lemma 1 (Discrete estimates) Let $\mathscr{D}$ be an admissible space-time discretization matching Definition 3. Assume that Hypotheses 1 and the continuity and coercivity properties (P2) and (P3) hold. Then, there exist $C_{1}, C_{2}, C_{3}, C_{4}>0$, depending on $\Omega, T, \zeta_{3}, \zeta_{4}, \beta_{0}, \varrho_{1}, \varrho_{2}, g, \bar{\lambda}_{i}$ with $i=1,2, \underline{\lambda}, \bar{\lambda}, L_{\psi}, C_{1, \psi}, C_{2, \psi}, s^{+}$ $, s^{-}, u_{\mathrm{init}}, \hat{C}_{1}$ and $C_{\chi}$ with $\chi=f_{1} \lambda_{\mathrm{T}}$ and $\chi=1$ such that any discrete solution $(p, u) \in\left[H_{\mathscr{T}}(\Omega \times(0, T))\right]^{2}$ of problem 28) satisfies

$$
\begin{aligned}
\sup _{t \in[0, T]}\|p(t)\|_{\mathcal{T}} & \leq C_{1}, \\
\|\psi(u)\|_{\mathscr{T}} & \leq C_{2}, \\
\sup _{t \in[0, T[}\|\Psi(u(t))\|_{L^{1}(\Omega)} & \leq C_{3}, \\
\sup _{t \in[0, T[}\|u(t)\|_{L^{2}(\Omega)} & \leq C_{4} .
\end{aligned}
$$

Proof Let $n \in \llbracket 0, N-1 \rrbracket$. Multiplying equation $28 \mathrm{~b}$ by $p_{K}^{(n+1)}$ and summing it over $K \in \mathcal{T}$ give $T_{p, 1}=T_{p, 2}+T_{p, 3}$ with

$$
\begin{aligned}
T_{p, 1} & =-\sum_{K \in \mathcal{T}} \sum_{\sigma \in \mathcal{E}_{K}} \lambda_{\mathrm{T}}\left(u_{1, \sigma}^{(n+1)}\right) F_{K, \sigma}\left(p^{(n+1)}, p^{(n+1)}\right) p_{K}^{(n+1)}, \\
T_{p, 2} & =-\sum_{K \in \mathcal{T}} \sum_{\sigma \in \mathcal{E}_{K}} \lambda_{\mathrm{T}}\left(u_{1, \sigma}^{(n+1)}\right) \varrho_{f}\left(u_{0, \sigma}^{(n+1)}\right) G_{K, \sigma} p_{K}^{(n+1)}, \\
T_{p, 3} & =\sum_{K \in \mathcal{T}}|K|\left(s_{K}^{+}-s_{K}^{-}\right) p_{K}^{(n+1)} .
\end{aligned}
$$

Thanks to the coercivity assumption (34), we obtain

$$
T_{p, 1} \geq \hat{C}_{1}\left\|p^{(n+1)}\right\|_{\mathcal{T}}^{2}
$$

By using Lemma 12 with $M(x, y)=\lambda_{\mathrm{T}}(x) \varrho_{f}(y)$, we deduce there exists a constant $C_{5}>0$ depending on $\underline{\lambda}, \bar{\lambda}, \bar{\lambda}_{1} \bar{\lambda}_{2}, \rho_{1}, \rho_{2}, g, \beta_{0}$ and $\Omega$ such that

$$
T_{p, 2} \leq C_{5}\left\|p^{(n+1)}\right\|_{\mathcal{T}}
$$

Using the Cauchy-Schwarz inequality and Proposition 4 of [34] yield

$$
\begin{aligned}
T_{p, 3} & \leq\left(\left\|s^{+}\right\|_{L^{2}(\Omega)}+\left\|s^{-}\right\|_{L^{2}(\Omega)}\right)\left\|p^{(n+1)}\right\|_{L^{2}(\Omega)} \\
& \leq\left(\left\|s^{+}\right\|_{L^{2}(\Omega)}+\left\|s^{-}\right\|_{L^{2}(\Omega)}\right) C_{6}\left\|p^{(n+1)}\right\|_{\mathcal{T}}
\end{aligned}
$$

where $C_{6}$ depends on $\Omega, \zeta_{3}$ and $\zeta_{4}$. The previous inequalities thus lead to

$$
\left\|p^{(n+1)}\right\|_{\mathcal{T}} \leq \frac{1}{\hat{C}_{1}}\left(C_{5}+C_{6}\left(\left\|s^{+}\right\|_{L^{2}(\Omega)}+\left\|s^{-}\right\|_{L^{2}(\Omega)}\right)\right) .
$$

Since this estimate is also valid for $n=-1$, we therefore have

$$
\sup _{t \in[0, T[}\|p(t)\|_{\mathcal{T}} \leq \frac{1}{\hat{C}_{1}}\left(C_{5}+C_{6}\left(\left\|s^{+}\right\|_{L^{2}(\Omega)}+\left\|s^{-}\right\|_{L^{2}(\Omega)}\right)\right),
$$

which gives 36 .

Multiplying equation (28a) by $\psi\left(u_{K}^{(n+1)}\right)$ and summing it up over $K \in \mathcal{T}$ results in $T_{\psi}=T_{\psi, 1}+$ 
266

$$
\begin{aligned}
T_{\psi} & =\sum_{K \in \mathcal{T}}|K| \frac{u_{K}^{(n+1)}-u_{K}^{(n)}}{\delta t^{\left(n+\frac{1}{2}\right)}} \psi\left(u_{K}^{(n+1)}\right)-\sum_{K \in \mathcal{T}} \sum_{\sigma \in \mathcal{E}_{K}} F_{K, \sigma}\left(\psi\left(u^{(n+1)}\right), \psi\left(u^{(n+1)}\right)\right) \psi\left(u_{K}^{(n+1)}\right), \\
T_{\psi, 1} & =-\sum_{K \in \mathcal{T}} \sum_{\sigma \in \mathcal{E}_{K}} f_{1}\left(u_{1, \sigma}^{(n+1)}\right) \mathbf{v}_{K, \sigma}^{(n+1)} \psi\left(u_{K}^{(n+1)}\right), \\
T_{\psi, 2} & =\sum_{K \in \mathcal{T}} \sum_{\sigma \in \mathcal{E}_{K}} f_{1}\left(u_{2, \sigma}^{(n+1)}\right) \lambda_{2}\left(u_{3, \sigma}^{(n+1)}\right)\left(\varrho_{2}-\varrho_{1}\right) G_{K, \sigma} \psi\left(u_{K}^{(n+1)}\right), \\
T_{\psi, 3}= & \sum_{K \in \mathcal{T}}|K|\left(f_{1}\left(c_{K}\right) s_{K}^{+}-f_{1}\left(u_{K}^{(n+1)}\right) s_{K}^{-}\right) \psi\left(u_{K}^{(n+1)}\right) .
\end{aligned}
$$

Using inequality (14) together with the coercivity property 34 with $\chi=1$, we obtain

$$
T_{\psi} \geq \sum_{K \in \mathcal{T}}|K| \frac{\Psi\left(u_{K}^{(n+1)}\right)-\Psi\left(u_{K}^{(n)}\right)}{\delta t^{\left(n+\frac{1}{2}\right)}}+\hat{C}_{1}\left\|\psi\left(u^{(n+1)}\right)\right\|_{\mathcal{T}}^{2} .
$$

Let us consider the term $T_{\psi, 1}$. Using the continuity property (33) with $\chi=f_{1} \lambda_{\mathrm{T}}$ and Lemma 12 with $M(x, y)=\left(f_{1} \lambda_{\mathrm{T}}\right)(x) \varrho_{f}(y)$, we get

$$
T_{\psi, 1} \leq C_{\chi}\left\|p^{(n+1)}\right\|_{\mathcal{T}}\left\|\psi\left(u^{(n+1)}\right)\right\|_{\mathcal{T}}+C_{7}\left\|\psi\left(u^{(n+1)}\right)\right\|_{\mathcal{T}} .
$$

Again, using Lemma 12 with $M(x, y)=f_{1}(x) \lambda_{2}(y)\left(\varrho_{2}-\varrho_{1}\right)$ gives

$$
T_{\psi, 2} \leq C_{8}\left\|\psi\left(u^{(n+1)}\right)\right\|_{\mathcal{T}}
$$

For the term $T_{\psi, 3}$ we proceed in the same way as previously for the pressure estimate and use Young's inequality, which leads to

$$
\begin{aligned}
T_{\psi, 3} & \leq\left(\left\|s^{+}\right\|_{L^{2}(\Omega)}+\left\|s^{-}\right\|_{L^{2}(\Omega)}\right)\left\|\psi\left(u^{(n+1)}\right)\right\|_{L^{2}(\Omega)} \\
& \leq \frac{\hat{C}_{1}}{4}\left\|\psi\left(u^{(n+1)}\right)\right\|_{\mathcal{T}}^{2}+\frac{1}{\hat{C}_{1}}\left(C_{6}\left(\left\|s^{+}\right\|_{L^{2}(\Omega)}+\left\|s^{-}\right\|_{L^{2}(\Omega)}\right)\right)^{2} .
\end{aligned}
$$

By combining these estimates we deduce that

$$
\begin{aligned}
\sum_{K \in \mathcal{T}}|K| \frac{\Psi\left(u_{K}^{(n+1)}\right)-\Psi\left(u_{K}^{(n)}\right)}{\delta t^{\left(n+\frac{1}{2}\right)}}+\hat{C}_{1}\left\|\psi\left(u^{(n+1)}\right)\right\|_{\mathcal{T}}^{2} \leq & C_{\chi}\left\|p^{(n+1)}\right\| \mathcal{T}\left\|\psi\left(u^{(n+1)}\right)\right\|_{\mathcal{T}}+\left(C_{7}+C_{8}\right)\left\|\psi\left(u^{(n+1)}\right)\right\|_{\mathcal{T}} \\
& +\frac{\hat{C}_{1}}{4}\left\|\psi\left(u^{(n+1)}\right)\right\|_{\mathcal{T}}^{2}+\frac{1}{\hat{C}_{1}}\left(C_{6}\left(\left\|s^{+}\right\|_{L^{2}(\Omega)}+\left\|s^{-}\right\|_{L^{2}(\Omega)}\right)\right)^{2} .
\end{aligned}
$$

Using again Young inequality and estimate (36), we deduce that there is a constant $\tilde{C}_{1}$ such that

$$
\sum_{K \in \mathcal{T}}|K| \frac{\Psi\left(u_{K}^{(n+1)}\right)-\Psi\left(u_{K}^{(n)}\right)}{\delta t^{\left(n+\frac{1}{2}\right)}}+\frac{\hat{C}_{1}}{4}\left\|\psi\left(u^{(n+1)}\right)\right\|_{\mathcal{T}}^{2} \leq \tilde{C}_{1} .
$$

Multiplying this inequality by $\delta t^{\left(n+\frac{1}{2}\right)}$, summing over $n=0, \cdots, \ell-1$ with $\ell \in \llbracket 1, N \rrbracket$, and using inequalities $\sqrt{12}$ to obtain

$$
\left\|\Psi\left(u^{(\ell)}\right)\right\|_{L^{1}(\Omega)}+\frac{\hat{C}_{1}}{4} \int_{0}^{t^{(\ell)}}\|\psi(u(s))\|_{\mathcal{T}}^{2} d s \leq \tilde{C}_{1} T+\frac{L_{\psi}}{2}\left\|u^{(0)}\right\|_{L^{2}(\Omega)}^{2} .
$$

Since 45 is valid for all $\ell \in \llbracket 1, N \rrbracket$, we deduce, for $\ell=N$,

$$
\|\psi(u)\|_{\mathscr{T}} \leq\left(\frac{4}{\hat{C}_{1}}\left(\tilde{C}_{1} T+\frac{L_{\psi}}{2}\left\|u^{(0)}\right\|_{L^{2}(\Omega)}^{2}\right)\right)^{\frac{1}{2}}
$$


which gives (37).

If $t \in\left[t^{(0)}, t^{(1)}\left[\right.\right.$ then $u(t)=u^{(0)}$ and hence (12) gives

$$
\|\Psi(u(t))\|_{L^{1}(\Omega)}=\left\|\Psi\left(u^{(0)}\right)\right\|_{L^{1}(\Omega)} \leq \frac{L_{\psi}}{2}\left\|u^{(0)}\right\|_{L^{2}(\Omega)}^{2} .
$$

If $t \in\left[t^{(1)}, T\left[\right.\right.$ then there exists $\ell \in \llbracket 1, N \rrbracket$ such that $t \in\left[t^{(\ell)}, t^{(\ell+1)}\left[\right.\right.$ and hence $u(t)=u^{(\ell)}$ and thanks to 45 we get

$$
\|\Psi(u(t))\|_{L^{1}(\Omega)} \leq \tilde{C}_{1} T+\frac{L_{\psi}}{2}\left\|u^{(0)}\right\|_{L^{2}(\Omega)}^{2} .
$$

From the two previous inequalities we deduce that

$$
\sup _{t \in[0, T[}\|\Psi(u(t))\|_{L^{1}(\Omega)} \leq \tilde{C}_{1} T+\frac{L_{\psi}}{2}\left\|u^{(0)}\right\|_{L^{2}(\Omega)}^{2},
$$

which gives (38).

Finally, thanks to (13) and (47), we deduce (39).

Lemma 2 (Discrete $H^{-1}$-estimate) Let $\mathscr{D}$ be a space-time discretization matching Definition 3. Assume that Hypotheses 1 and the continuity and coercivity properties, (P2) and (P3), hold. Then, there exists $C_{9}>0$, depending on $\Omega, T, \zeta_{3}, \zeta_{4}, \beta_{0}, \varrho_{1}, \varrho_{2}, g, \bar{\lambda}_{i}$ with $i=1,2, \underline{\lambda}, \bar{\lambda}, L_{\psi}, s^{+}, s^{-}, u_{\text {init }}, \hat{C}_{1}$, and $C_{\chi}$ with $\chi=f_{1} \lambda_{\mathrm{T}}$ and $\chi=1$ such that any discrete solution $u \in H_{\mathscr{T}}(\Omega \times(0, T))$ of problem (28) satisfies

$$
\left\|\delta_{t} u\right\|_{-1, \mathscr{T}} \leq C_{9} .
$$

Proof For any $w \in H_{\mathcal{T}}(\Omega)$, we deduce, from $28 \mathrm{a}$, that

$$
\begin{aligned}
\left|\sum_{K \in \mathcal{T}}\right| K\left|\frac{u_{K}^{(n+1)}-u_{K}^{(n)}}{\delta t^{\left(n+\frac{1}{2}\right)}} w_{K}\right| \leq & \left|\sum_{K \in \mathcal{T}} \sum_{\sigma \in \mathcal{E}_{K}} f_{1}\left(u_{1, \sigma}^{(n+1)}\right) \mathbf{v}_{K, \sigma}^{(n+1)} w_{K}\right| \\
& +\left|\sum_{K \in \mathcal{T}} \sum_{\sigma \in \mathcal{E}_{K}} f_{1}\left(u_{2, \sigma}^{(n+1)}\right) \lambda_{2}\left(u_{3, \sigma}^{(n+1)}\right)\left(\varrho_{1}-\varrho_{2}\right) G_{K, \sigma} w_{K}\right| \\
& +\left|\sum_{K \in \mathcal{T}} \sum_{\sigma \in \mathcal{E}_{K}} F_{K, \sigma}\left(\psi\left(u^{(n+1)}\right), \psi\left(u^{(n+1)}\right)\right) w_{K}\right| \\
& +\left|\sum_{K \in \mathcal{T}}\left(f_{1}\left(c_{K}\right) s_{K}^{+}-f_{1}\left(u_{K}^{(n+1)}\right) s_{K}^{-}\right) w_{K}\right| .
\end{aligned}
$$

The four terms on the right hand side of 49 ) can be bounded using the same techniques as in the proof of Lemma 1 This provides the existence of a constant $C_{10}>0$ such that, for all $w \in H_{\mathcal{T}}(\Omega)$,

$$
\left|\int_{\Omega}\left(\delta_{t} u\right)^{(n)}(x) w(x) d x\right| \leq C_{10}\left(\left\|p^{(n+1)}\right\|_{\mathcal{T}}+\left\|\psi\left(u^{(n+1)}\right)\right\|_{\mathcal{T}}+\left\|s^{+}\right\|_{L^{2}(\Omega)}+\left\|s^{-}\right\|_{L^{2}(\Omega)}+1\right)\|w\|_{\mathcal{T}},
$$

from which we deduce that

$$
\left\|\left(\delta_{t} u\right)^{(n)}\right\|_{-1, \mathcal{T}} \leq C_{10}\left(\left\|p^{(n+1)}\right\|_{\mathcal{T}}+\left\|\psi\left(u^{(n+1)}\right)\right\|_{\mathcal{T}}+\left\|s^{+}\right\|_{L^{2}(\Omega)}+\left\|s^{-}\right\|_{L^{2}(\Omega)}+1\right) .
$$

Squaring both sides of the inequality above, multiplying it by $\delta t^{\left(n+\frac{1}{2}\right)}$, and summing up over $n=$ $0, \ldots, N-1$, results in

$$
\left\|\left(\delta_{t} u\right)\right\|_{-1, \mathscr{T}}^{2} \leq 5 C_{10}^{2}\left(\|p\|_{\mathscr{T}}^{2}+\|\psi(u)\|_{\mathscr{T}}^{2}+T\left(\left\|s^{+}\right\|_{L^{2}(\Omega)}^{2}+\left\|s^{-}\right\|_{L^{2}(\Omega)}^{2}+1\right)\right) .
$$

Then, thanks to Lemma 1 , we deduce that there exists $C_{9}>0$, depending on $\Omega, T, \zeta_{3}, \zeta_{4}, \beta_{0}, \varrho_{1}$, $\varrho_{2}, g, \bar{\lambda}_{i}$ with $i=1,2, \underline{\lambda}, \bar{\lambda}, L_{\psi}, s^{+}, s^{-}, u_{\text {init }}$, and $C_{\chi}$ with $\chi=f_{1} \lambda_{\mathrm{T}}$ and $\chi=1$ such that

$$
\left\|\delta_{t} u\right\|_{-1, \mathscr{T}} \leq C_{9} .
$$


Lemma 3 (Estimate on the time translates) Let $\mathscr{D}$ be a space-time discretization matching Definition 3. Assume that Hypotheses 1 and the continuity and coercivity properties, (P2) and (P3), hold. Then, there exists $C_{11}>0$ depending on $\Omega, T, \zeta_{3}, \zeta_{4}, \beta_{0}, \varrho_{1}, \varrho_{2}, g, \bar{\lambda}_{i}$ with $i=1,2, \underline{\lambda}, \bar{\lambda}, L_{\psi}, s^{+}, s^{-}, u_{\text {init }}, \hat{C}_{1}$ and $C_{\chi}$ with $\chi=f_{1} \lambda_{\mathrm{T}}$ and $\chi=1$, such that any discrete solution $u \in H_{\mathscr{T}}(\Omega \times(0, T))$ of problem $(28)$ satisfies

$$
\left.\|\psi(u)(\cdot, \cdot+\tau)-\psi(u)\|_{L^{2}(\Omega \times(0, T-\tau))} \leq C_{11} \sqrt{\tau}, \quad \forall \tau \in\right] 0, T[.
$$

Proof Thanks to Lemma 2 and to the estimate (37) of Lemma 1 , (50) can be obtained by following the proof of Lemma 3.11 in 8 .

Lemma 4 ( $H^{-1}$-estimate) Let $\mathscr{D}$ be a space-time discretization matching Definition 3 . Assume that Hypotheses 1 and the continuity and coercivity properties, (P2) and (P3), hold. Then, there exists $C_{12}>0$, depending on $\Omega, T, \alpha, \zeta_{1}, \zeta_{3}, \zeta_{4}, \beta_{0}, \varrho_{1}, \varrho_{2}, g, \bar{\lambda}_{i}$ with $i=1,2, \underline{\lambda}, \bar{\lambda}, L_{\psi}, s^{+}, s^{-}, u_{\text {init }}, \hat{C}_{1}$ and $C_{\chi}$ with $\chi=f_{1} \lambda_{\mathrm{T}}$ and $\chi=1$, such that any discrete solution $u \in H_{\mathscr{T}}(\Omega \times(0, T))$ of problem (28) satisfies

$$
\left\|\delta_{t} u\right\|_{L^{2}\left(0, T ; H_{0}^{-1}(\Omega)\right)} \leq C_{12} .
$$

Proof For any $w \in H_{0}^{1}(\Omega)$ and $n \in \llbracket 0, N \rrbracket$, using Lemma 10 , we have

$$
\begin{aligned}
\left|\int_{\Omega}\left(\delta_{t} u\right)^{(n)} w \mathrm{~d} x\right| & =\left|\int_{\Omega}\left(\delta_{t} u\right)^{(n)} w_{\mathcal{T}} \mathrm{d} x\right| \\
& \leq\left\|\left(\delta_{t} u\right)^{(n)}\right\|_{-1, \mathcal{T}}\left\|w_{\mathcal{T}}\right\|_{\mathcal{T}} \\
& \leq C_{16}\left\|\left(\delta_{t} u\right)^{(n)}\right\|_{-1, \mathcal{T}}\|w\|_{H_{0}^{1}(\Omega)} .
\end{aligned}
$$

We thus deduce that

$$
\left\|\delta_{t} u\right\|_{L^{2}\left(0, T ; H_{0}^{-1}(\Omega)\right)} \leq C_{16}\left\|\left(\delta_{t} u\right)\right\|_{-1, \mathscr{T}}
$$

and conclude the proof thanks to Lemma 2

Lemma 5 ( $L^{2}$-estimate on the dual mesh $\left.\Delta\right)$ Let $\mathscr{D}$ be a space-time discretization matching Definition 3. Assume that Hypotheses 1 and the continuity and coercivity properties, [(P2) and (P3), hold. Then, there exists $C_{13}>0$, depending on $\Omega, T, \zeta_{3}, \zeta_{4}, \beta_{0}, \varrho_{1}, \varrho_{2}, g, \bar{\lambda}_{i}$ with $i=1,2, \underline{\lambda}, \bar{\lambda}, L_{\psi}, s^{+}, s^{-}, u_{\text {init }}, \hat{C}_{1}$ and $C_{\chi}$ with $\chi=f_{1} \lambda_{\mathrm{T}}$ and $\chi=1$, such that any discrete solution $u \in H_{\mathscr{T}}(\Omega \times(0, T))$ of problem 28) satisfies

$$
\|\psi(u)-\psi(v)\|_{L^{2}(\Omega \times(0, T))} \leq C_{13} h_{\mathcal{D}},
$$

for all $v \in H_{\mathscr{E}}(\Omega \times(0, T))$ such that for all $n \in \llbracket 0, N \rrbracket, \sigma \in \mathcal{E}, v_{\sigma}^{(n)} \in\left[\min \left\{u_{K}^{(n)}, u_{L}^{(n)}\right\}, \max \left\{u_{K}^{(n)}, u_{L}^{(n)}\right\}\right]$ if $\mathcal{T}_{\sigma}=\{K, L\}$ otherwise if $\mathcal{T}_{\sigma}=\{K\}, v_{\sigma}^{(n)} \in\left[\min \left\{u_{K}^{(n)}, 0\right\}, \max \left\{u_{K}^{(n)}, 0\right\}\right]$.

Proof We have

$$
\begin{aligned}
\|\psi(u)-\psi(v)\|_{L^{2}(\Omega \times(0, T))}^{2} & =\sum_{n=0}^{N} \delta t^{n+\frac{1}{2}}\left\|\psi\left(u^{(n)}\right)-\psi\left(v^{(n)}\right)\right\|_{L^{2}(\Omega)}^{2} \\
& =\sum_{n=0}^{N} \delta t^{n+\frac{1}{2}} \sum_{K \in \mathcal{T}} \sum_{\sigma \in \mathcal{E}_{K}}\left|\Delta_{K, \sigma}\right|\left(\psi\left(u_{K}^{(n)}\right)-\psi\left(v_{\sigma}^{(n)}\right)\right)^{2} .
\end{aligned}
$$

Since $\psi$ is a monotone function, we notice that for all $K \in \mathcal{T}$ and $\sigma \in \mathcal{E}_{K}$ :

- if $\sigma \in \mathcal{E}_{\text {int }}$ with $\mathcal{T}_{\sigma}=\{K, L\}$ then

$$
\begin{aligned}
\left|\psi\left(u_{K}^{(n)}\right)-\psi\left(v_{\sigma}^{(n)}\right)\right| & \leq\left|\psi\left(u_{K}^{(n)}\right)-\psi\left(u_{L}^{(n)}\right)\right| \\
& =\frac{d_{K, \sigma}+d_{L, \sigma}}{d_{K, \sigma}}\left|\psi\left(u_{K}^{(n)}\right)-\gamma_{\sigma}\left(\psi\left(u^{(n)}\right)\right)\right|,
\end{aligned}
$$


- if $\sigma \in \mathcal{E}_{\text {ext }}$ then $\left|\psi\left(u_{K}^{(n)}\right)-\psi\left(v_{\sigma}^{(n)}\right)\right| \leq\left|\psi\left(u_{K}^{(n)}\right)\right|$.

By using the mesh regularity, we then deduce

$$
\begin{aligned}
\|\psi(u)-\psi(v)\|_{L^{2}(\Omega \times(0, T))}^{2} & \leq \frac{2}{\zeta_{4}} \sum_{n=0}^{N} \delta t^{n+\frac{1}{2}} \sum_{K \in \mathcal{T}} \sum_{\sigma \in \mathcal{E}_{K}}\left|\Delta_{K, \sigma}\right|\left(\psi\left(u_{K}^{(n)}\right)-\gamma_{\sigma}\left(\psi\left(u^{(n)}\right)\right)\right)^{2} \\
& =\frac{2}{\zeta_{4}} \sum_{n=0}^{N} \delta t^{n+\frac{1}{2}} \sum_{K \in \mathcal{T}} \sum_{\sigma \in \mathcal{E}_{K}} \frac{|\sigma| d_{K, \sigma}}{d}\left(\psi\left(u_{K}^{(n)}\right)-\gamma_{\sigma}\left(\psi\left(u^{(n)}\right)\right)\right)^{2} \\
& \leq \frac{2 h_{\mathcal{D}}^{2}}{\zeta_{4} d} \sum_{n=0}^{N} \delta t^{n+\frac{1}{2}} \sum_{K \in \mathcal{T}} \sum_{\sigma \in \mathcal{E}_{K}} \frac{|\sigma|}{d_{K, \sigma}}\left(\psi\left(u_{K}^{(n)}\right)-\gamma_{\sigma}\left(\psi\left(u^{(n)}\right)\right)\right)^{2} \\
& =\frac{2 h_{\mathcal{D}}^{2}}{\zeta_{4} d}\|\psi(u)\|_{\mathscr{T}}^{2} .
\end{aligned}
$$

Finally, using the estimate 37 of Lemma 1 we obtain that there exists a constant $C_{13}>0$ such that $\|\psi(u)-\psi(v)\|_{L^{2}(\Omega \times(0, T))} \leq C_{13} h_{\mathcal{D}}$.

\subsection{Existence of discrete solutions}

Proposition 1 (Existence of discrete solutions) Let $\mathscr{D}$ be a space-time discretization matching Definition 3. Assume that Hypotheses 1 and the continuity and coercivity properties, (P2) and (P3), hold. For all $n \in \llbracket 0, N-1 \rrbracket$, there exists at least one solution to the equations (28).

Proof Let us take $n \in \llbracket 0, N-1 \rrbracket$ and let us introduce the following open bounded subset of $\mathbb{R}^{n_{\mathcal{T}}} \times \mathbb{R}^{n_{\mathcal{T}}}$

$$
\omega=\left\{\left(p_{\mathcal{T}}^{(n+1)}, u_{\mathcal{T}}^{(n+1)}\right) \in \mathbb{R}^{n \mathcal{T}} \times \mathbb{R}^{n \mathcal{T}} \mid\left\|p^{(n+1)}\right\|_{\mathcal{T}}<C_{1}+1 \text { and }\left\|u^{(n+1)}\right\|_{L^{2}(\Omega)}<C_{4}+1\right\}
$$

and the application $\eta_{\nu}$ defined over $\omega$ and for all $\nu \in[0,1]$, by

$$
\eta_{\nu}:\left\{\begin{array}{l}
\mathbb{R}^{n_{\mathcal{T}}} \times \mathbb{R}^{n_{\mathcal{T}}} \rightarrow \mathbb{R}^{n_{\mathcal{T}}} \times \mathbb{R}^{n_{\mathcal{T}}} \\
\left(p_{\mathcal{T}}^{(n+1)}, u_{\mathcal{T}}^{(n+1)}\right) \mapsto\left(\eta_{\nu, 1}, \eta_{\nu, 2}\right)
\end{array}\right.
$$

where, for all $K \in \mathcal{T}$,

$$
\begin{aligned}
\left(\eta_{\nu, 1}\right)_{K}= & -\nu\left(\sum_{\sigma \in \mathcal{E}_{K}} \lambda_{\mathrm{T}}\left(u_{1, \sigma}^{(n+1)}\right)\left(F_{K, \sigma}\left(p^{(n+1)}, p^{(n+1)}\right)-\varrho_{f}\left(u_{0, \sigma}^{(n+1)}\right) G_{K, \sigma}\right)+|K|\left(s_{K}^{+}-s_{K}^{-}\right)\right) \\
& +(1-\nu)|K| p_{K}^{(n+1)}, \\
\left(\eta_{\nu, 2}\right)_{K}= & \nu\left(|K| \frac{u_{K}^{(n+1)}-u_{K}^{(n)}}{\delta t^{\left(n+\frac{1}{2}\right)}}+\sum_{\sigma \in \mathcal{E}_{K}}\left(\begin{array}{l}
f_{1}\left(u_{1, \sigma}^{(n+1)}\right) \mathbf{v}_{K, \sigma}^{(n+1)} \\
+f_{1}\left(u_{2, \sigma}^{(n+1)}\right) \lambda_{2}\left(u_{3, \sigma}^{(n+1)}\right)\left(\varrho_{1}-\varrho_{2}\right) G_{K, \sigma} \\
-F_{K, \sigma}\left(\psi\left(u^{(n+1)}\right), \psi\left(u^{(n+1)}\right)\right)
\end{array}\right)\right) \\
& -\nu|K|\left(f_{1}\left(c_{K}\right) s_{K}^{+}-f_{1}\left(u_{K}^{(n+1)}\right) s_{K}^{-}\right) \\
& +(1-\nu)|K| u_{K}^{(n+1)} .
\end{aligned}
$$

$\eta_{\nu}$ is continuous with respect to $\nu, p_{\mathcal{T}}^{(n+1)}$ and $u_{\mathcal{T}}^{(n+1)}$. Thanks to 14 used with $s_{1}=0$ and $[12$, we get that for all $s \in \mathbb{R}$,

$$
s \psi(s) \geq 0 .
$$

Then proceeding in the same way as in the proof of Lemma 1 and thanks to (53), we deduce that

$$
\forall \nu \in[0,1], \mathbf{0}_{\mathbb{R}^{2 n_{\mathcal{T}}}} \notin \eta_{\nu}(\partial \omega) .
$$


The topological degree $d\left(\eta_{\nu}, \omega, \mathbf{0}_{\mathbb{R}^{2 n} \mathcal{T}}\right)$ is therefore well defined. For $\nu=0$, the associated system, $\eta_{\nu}=\mathbf{0}_{\mathbb{R}^{2 n} \mathcal{T}}$, admits one solution. Indeed, we have $p_{\mathcal{T}}^{(n+1)}=\mathbf{0}_{\mathbb{R}^{n} \mathcal{T}}$ and $u_{\mathcal{T}}^{(n+1)}=\mathbf{0}_{\mathbb{R}^{n} \mathcal{T}}$ and both solutions belong to $\omega$. Since the degree is homotopy invariant, we have

$$
\forall \nu \in[0,1], d\left(\eta_{\nu}, \omega, \mathbf{0}_{\mathbb{R}^{2 n} \mathcal{T}}\right)=d\left(\eta_{0}, \omega, \mathbf{0}_{\mathbb{R}^{2 n} \mathcal{T}}\right) \neq 0 .
$$

As, for $\nu=1$, the system $\eta_{\nu}=\mathbf{0}_{\mathbb{R}^{2 n} \mathcal{T}}$ corresponds to 28), the previous relation guarantees the existence of a solution in $\omega$.

\subsection{Convergence proof}

Before proving the convergence of the discrete scheme in this section, we present a compactness result and establish some further estimates.

Lemma 6 (Compactness of approximate solution) Let $\mathscr{D}_{m}$ be a sequence of space-time discretizations matching Definition 蚱 and $\left(p_{m}, u_{m}\right) \in\left[H_{\mathscr{T}}(\Omega \times(0, T))\right]^{2}$ be a discrete solution of problem (28). Let us assume that Hypotheses 1 and the continuity and coercivity properties, (P2) and (P3), hold, then, up to a subsequence (still denoted with the subindex $m$ ), we have the following results:

(i) $\psi\left(u_{m}\right)$ converges in $L^{2}(\Omega \times] 0, T[)$ to some $\psi(\bar{u}) \in L^{2}\left(0, T ; H_{0}^{1}(\Omega)\right.$ ), (and therefore $u_{m}$ converges in $L^{2}(\Omega \times] 0, T[)$ to $\left.\bar{u}\right)$,

(ii) for any $\Upsilon_{m} \in \mathscr{R}_{\mathcal{E}_{m}}, \widetilde{\nabla}_{\mathcal{D}_{m}, \Upsilon_{m}} \psi\left(u_{m}\right)$ weakly converges in $L^{2}(\Omega \times] 0, T[)^{d}$ to $\nabla \psi(\bar{u})$,

(iii) $p_{m}$ weakly converges in $L^{2}(\Omega \times] 0, T[)$ to some $\bar{p} \in L^{2}\left(0, T ; H_{0}^{1}(\Omega)\right)$,

(iv) for any $\Upsilon_{m} \in \mathscr{R}_{\mathcal{E}_{m}}, \widetilde{\nabla}_{\mathcal{D}_{m}, \Upsilon_{m}} p_{m}$ weakly converges in $L^{2}(\Omega \times] 0, T[)^{d}$ to $\nabla \bar{p}$,

(v) $\left(\delta_{t} u\right)_{m}$ weak-太 converges in $L^{2}\left(0, T ; H_{0}^{-1}(\Omega)\right)$ to $\partial_{t} \bar{u}$,

(vi) $u_{m}$ weakly converges in $L^{2}(\Omega)$ uniformly on $[0, T]$ to $\bar{u}$,

52 (vii) for $T_{m} \rightarrow T$,

$$
\liminf _{m \rightarrow \infty} \int_{\Omega} \Psi\left(u_{m}\left(T_{m}\right)\right) \geq \int_{\Omega} \Psi(\bar{u}(T)) .
$$

${ }_{353}\left(\right.$ viii) $\psi\left(\left(u_{m}\right)_{i}\right), i \in\{1,2,3\}$, strongly converge to $\psi(\bar{u}) \in L^{2}\left(0, T ; H_{0}^{1}(\Omega)\right)$ in $L^{2}(\Omega \times] 0, T[)$, (and therefore $354 \quad\left(u_{m}\right)_{i}$ converge in $L^{2}(\Omega \times] 0, T[)$ to $\left.\bar{u}\right)$.

Proof We first define $\tilde{\psi}_{m}$ by $\tilde{\psi}_{m}=\psi\left(u_{m}\right)$ a.e. on $\left.\Omega \times\right] 0, T\left[\right.$ and $\tilde{\psi}_{m}=0$ a.e. on $\left.\mathbb{R}^{d+1} \backslash \Omega \times\right] 0, T$. Following the proof of Lemma 3.3 in [21], using the fact that, for all $v \in H_{\mathcal{T}}(\Omega)$

$$
\frac{\left(v_{K}-v_{L}\right)^{2}}{d_{K, \sigma}+d_{L, \sigma}} \leq \frac{\left(\gamma_{\sigma} v-v_{K}\right)^{2}}{d_{K, \sigma}}+\frac{\left(\gamma_{\sigma} v-v_{L}\right)^{2}}{d_{L, \sigma}},
$$

and (37), we can prove that there exists a constant $C_{14}>0$ only depending on $\Omega$ s.t.

$$
\left\|\tilde{\psi}_{m}(\cdot+\eta, \cdot)-\tilde{\psi}_{m}\right\|_{L^{2}\left(\mathbb{R}^{d+1}\right)}^{2} \leq C_{2}^{2}\|\eta\|\left(\|\eta\|+C_{14} h_{\mathcal{D}_{m}}\right), \quad \forall \eta \in \mathbb{R}^{d} .
$$

From (50), (39), and the Lipschitz property of $\psi$, we also deduce that

$$
\left\|\tilde{\psi}_{m}(\cdot, \cdot+\tau)-\tilde{\psi}_{m}\right\|_{L^{2}\left(\mathbb{R}^{d+1}\right)}^{2} \leq|\tau|\left(|\tau| C_{11}^{2}+2 L_{\psi}^{2} C_{4}^{2}\right), \quad \forall \tau \in \mathbb{R} .
$$

Thus, with (54) and (55), an application of the Kolmogorov theorem gives that $\psi\left(u_{m}\right)$ strongly converges to some $\Phi$ in $L^{2}(\Omega \times] 0, T[)$. Therefore, $\psi\left(u_{m}\right)$ strongly converges to $\psi(\bar{u})$ with $\bar{u}=\psi^{-1}(\Phi)$. Then, thanks to the assumptions made on $\psi$ (see Hypotheses 1]) and by using Lemma 7.1 in [4] with $g=\psi^{-1}$, we deduce that $u_{m}$ also strongly converges to $\bar{u}$.

For any $\Upsilon_{m} \in \mathscr{R}_{\mathcal{E}_{m}}$, using (37), the equivalence of the norms $\|\cdot\|_{\mathscr{T}}$ and $\|\cdot\|_{\mathscr{T}, \Upsilon_{m}}$ (see Lemma 1 in 34]) and a straightforward adaptation of Lemma 4.3 in 22 to time dependent problems, we deduce that there exists $\mathbf{G}$ such that $\widetilde{\nabla}_{\mathcal{D}_{m}, \Upsilon_{m}} \psi\left(u_{m}\right)$ weakly converges in $L^{2}(\Omega \times] 0, T[)^{d}$ to $\mathbf{G}=\nabla \Phi$.

From (36) and Proposition 4 in [34], we have that $p_{m}$ is also bounded in $L^{2}(\Omega \times] 0, T[)$ and thus weakly converges to some limit $\bar{p}$. Again, for any $\Upsilon_{m} \in \mathscr{R}_{\mathcal{E}_{m}}$, using (36), the equivalence of the norms 
$\|\cdot\|_{\mathscr{T}}$ and $\|\cdot\|_{\mathscr{T}, \Upsilon_{m}}$ and Lemma 4.3 in $\underline{22}$, we deduce that $\bar{p} \in L^{2}\left(0, T ; H_{0}^{1}(\Omega)\right)$ and that $\widetilde{\nabla}_{\mathcal{D}_{m}, Y_{m}} p_{m}$ weakly converges in $L^{2}(\Omega \times] 0, T[)^{d}$ to $\nabla \bar{p}$.

From (51), we deduce that $\left(\delta_{t} u\right)_{m}$ weak- $\star$ converges in $L^{2}\left(0, T ; H_{0}^{-1}(\Omega)\right)$ to some $U$. In the same way as in the proof of Theorem 4.18 in [17] and by using the strong convergence of $u_{m}$ one can show that $U=\partial_{t} \bar{u}$.

Statement (vi) is obtained by using (39), (51) and Theorem 4.19 of [17, whereas (vii) is a consequence of (vi) the convexity of $\Psi$, and Lemma D.11 of [17. Finally, from (52) and (i) we obtain (viii)

Lemma 7 Let $\mathcal{D}$ be a space discretization matching Definition $\Omega: m: \mathbb{R} \times \mathbb{R} \mapsto \mathbb{R}$ a bounded function and $\Upsilon$ the trace reconstruction operator obtained by taking $\theta_{K, \sigma}=\delta_{K, \sigma}$ (see Definition 5 and equation (30)). For all $(u, v) \in\left[H_{\mathcal{T}}(\Omega)\right]^{2}$ and $\left(u_{1}, u_{2}\right) \in\left[H_{\Delta}(\Omega)\right]^{2}$ there exists a constant $\bar{C}_{1 m}>0$ such that

$\left|-\sum_{K \in \mathcal{T}} \sum_{\sigma \in \mathcal{E}_{K}} m\left(u_{1, \sigma}, u_{2, \sigma}\right) G_{K, \sigma} v_{K}-\int_{\Omega} m(u, u) \Lambda \mathbf{g} \cdot \widetilde{\nabla}_{\mathcal{D}, \Upsilon} v \mathrm{~d} x\right| \leq \bar{C}_{1 m}\left\|m\left(u_{1}, u_{2}\right)-m(u, u)\right\|_{L^{2}(\Omega)}\|v\|_{\mathcal{T}}$.

Proof Let $I=-\sum_{K \in \mathcal{T}} \sum_{\sigma \in \mathcal{E}_{K}} m\left(u_{1, \sigma}, u_{2, \sigma}\right) G_{K, \sigma} v_{K}$. Inserting the definition of $G_{K, \sigma}$ and by rearrange of terms, we deduce that

$$
I=\sum_{K \in \mathcal{T}} \sum_{\sigma \in \mathcal{E}_{K}}|\sigma| m\left(u_{1, \sigma}, u_{2, \sigma}\right) \Lambda_{K} \mathbf{n}_{K, \sigma} \cdot \mathbf{g}\left(\Upsilon_{\sigma} v-v_{K}\right) .
$$

We thus get $I=I_{1}+I_{2}$ with

$$
\begin{aligned}
I_{1} & =\sum_{K \in \mathcal{T}} \sum_{\sigma \in \mathcal{E}_{K}}|\sigma|\left(m\left(u_{1, \sigma}, u_{2, \sigma}\right)-m\left(u_{K}, u_{K}\right)\right) \Lambda_{K} \mathbf{n}_{K, \sigma} \cdot \mathbf{g}\left(\Upsilon_{\sigma} v-v_{K}\right), \\
I_{2} & =\sum_{K \in \mathcal{T}} \sum_{\sigma \in \mathcal{E}_{K}}|\sigma| m\left(u_{K}, u_{K}\right) \Lambda_{K} \mathbf{n}_{K, \sigma} \cdot \mathbf{g}\left(\Upsilon_{\sigma} v-v_{K}\right)=\int_{\Omega} m(u, u) \Lambda \mathbf{g} \cdot \widetilde{\nabla}_{\mathcal{D}, \Upsilon} v \mathrm{~d} x .
\end{aligned}
$$

Thanks to Cauchy-Schwarz inequality and the the equivalence of the discrete norms (see Lemma 1 in 34) we infer

$$
\begin{aligned}
I_{1} & \leq d \beta_{0}|g|\left(\sum_{K \in \mathcal{T}} \sum_{\sigma \in \mathcal{E}_{K}}\left|\Delta_{K, \sigma}\right|\left(m\left(u_{1, \sigma}, u_{2, \sigma}\right)-m\left(u_{K}, u_{K}\right)\right)^{2}\right)^{\frac{1}{2}}\|v\|_{\mathcal{T}, \Upsilon} \\
& \leq d \frac{2 \beta_{0}}{\zeta_{4}}|g|\left\|m\left(u_{1}, u_{2}\right)-m(u, u)\right\|_{L^{2}(\Omega)}\|v\|_{\mathcal{T}} .
\end{aligned}
$$

Lemma 8 (Error estimate for the strong convergence) Let $\mathscr{D}$ be a space-time discretization matching Definition 3 Let us assume that Hypotheses 1 , the linearity, continuity and coercivity properties, (P1), (P2) and (P3), hold. Let $(p, u) \in\left[H_{\mathscr{T}}(\Omega \times(0, T))\right]^{2}$ be a discrete solution of problem (28) and $(q, v) \in\left[H_{\mathscr{T}}(\Omega \times(0, T))\right]^{2}$ two test functions. Then there exist $\bar{C}_{2}, \bar{C}_{3}>0$ such that,

$$
\begin{aligned}
\|p-q\|_{\mathscr{T}}^{2} \leq \bar{C}_{2}( & \bar{C}_{3}\left\|m_{0}\left(u_{1}, u_{0}\right)-m_{0}(u, u)\right\|_{L^{2}(\Omega \times(0, T))}^{2} \\
& +\int_{0}^{T} \int_{\Omega}\left(\lambda_{\mathrm{T}} \varrho_{f}\right)(u) \Lambda \mathrm{g} \cdot \widetilde{\nabla}_{\mathcal{D}, r}(p-q) \\
& +\int_{0}^{T} \int_{\Omega}\left(s^{+}-s^{-}\right)(p-q) \\
& \left.-\int_{0}^{T} a_{\mathcal{T}, \lambda_{\mathrm{T}}, u_{1}(t)}(p(t), q(t), p(t)-q(t))\right),
\end{aligned}
$$


with $m_{0}(x, y)=\lambda_{\mathrm{T}}(x) \varrho_{f}(y)$, and $\bar{C}_{4}, \bar{C}_{5}, \bar{C}_{6}, \bar{C}_{7}>0$ such that,

$$
\begin{aligned}
\|\psi(u)-v\|_{\mathscr{T}}^{2} \leq \bar{C}_{4}( & -\left(\left\|\Psi\left(u^{N}\right)\right\|_{L^{1}(\Omega)}-\left\|\Psi\left(u^{0}\right)\right\|_{L^{1}(\Omega)}\right)+\int_{0}^{T} \int_{\Omega} \delta_{t} u v \\
& +\bar{C}_{5}\left\|m_{1}\left(u_{1}, u_{0}\right)-m_{1}(u, u)\right\|_{L^{2}(\Omega \times(0, T))}^{2} \\
& +\bar{C}_{6}\left\|m_{2}\left(u_{2}, u_{3}\right)-m_{2}(u, u)\right\|_{L^{2}(\Omega \times(0, T))}^{2} \\
& +\bar{C}_{7}\|p-q\|_{\mathscr{T}}^{2} \\
& -\int_{0}^{T} a_{\mathcal{T}, \lambda_{\mathrm{T}} f_{1}, u_{1}(t)}(p(t), q(t), \psi(u(t))-v(t)) \\
& +\int_{0}^{T} \int_{\Omega}\left(f_{1} \lambda_{\mathrm{T}} \varrho_{f}+f_{1} \lambda_{2}\left(\varrho_{1}-\varrho_{2}\right)\right)(u) \Lambda \mathbf{g} \cdot \widetilde{\nabla}_{\mathcal{D}, r}(\psi(u)-v) \\
& +\int_{0}^{T} \int_{\Omega}\left(f_{1}(c) s^{+}-f_{1}(u) s^{-}\right)(\psi(u)-v) \\
& \left.-\int_{0}^{T} a_{\mathcal{T}, 1,1}(\psi(u(t)), v(t), \psi(u(t))-v(t))\right),
\end{aligned}
$$

with $m_{1}(x, y)=\left(f_{1} \lambda_{\mathrm{T}}\right)(x) \varrho_{f}(y)$ and $m_{2}(x, y)=f_{1}(x) \lambda_{2}(y)\left(\rho_{2}-\rho_{1}\right)$.

Proof Let $n \in \llbracket 0, N-1 \rrbracket$. We set

$$
T_{p, 1}=-\sum_{K \in \mathcal{T}} \sum_{\sigma \in \mathcal{E}_{K}} \lambda_{\mathrm{T}}\left(u_{1, \sigma}^{(n+1)}\right) F_{K, \sigma}\left(p^{(n+1)}, p^{(n+1)}-q^{(n+1)}\right)\left(p_{K}^{(n+1)}-q_{K}^{(n+1)}\right) .
$$

Thanks to assumption (34), with $\chi=\lambda_{\mathrm{T}}$ and $\alpha=u_{1}^{(n+1)}$, we first have

$$
T_{p, 1} \geq \hat{C}_{1}\left\|p^{(n+1)}-q^{(n+1)}\right\|_{\mathcal{T}}^{2} .
$$

After setting

$$
\tilde{T}_{p, 4}=-\sum_{K \in \mathcal{T}} \sum_{\sigma \in \mathcal{E}_{K}} \lambda_{\mathrm{T}}\left(u_{1, \sigma}^{(n+1)}\right) F_{K, \sigma}\left(p^{(n+1)}, q^{(n+1)}\right)\left(p_{K}^{(n+1)}-q_{K}^{(n+1)}\right),
$$

we observe that $T_{p, 1}=\tilde{T}_{p, 2}+\tilde{T}_{p, 3}-\tilde{T}_{p, 4}$. Then, by using Lemma 7 with $m_{0}(x, y)=\lambda_{\mathrm{T}}(x) \varrho_{f}(y)$, we get that there exists a constant $\bar{C}_{1 m_{0}}>0$ such that

$$
\begin{aligned}
\tilde{T}_{p, 2} \leq & \bar{C}_{1 m_{0}}\left\|m_{0}\left(u_{1}^{(n+1)}, u_{0}^{(n+1)}\right)-m_{0}\left(u^{(n+1)} u^{(n+1)}\right)\right\|_{L^{2}(\Omega)}\left\|p^{(n+1)}-q^{(n+1)}\right\|_{\mathcal{T}} \\
& +\int_{\Omega}\left(\lambda_{\mathrm{T}} \varrho_{f}\right)\left(u^{(n+1)}\right) \Lambda \mathbf{g} \cdot \widetilde{\nabla}_{\mathcal{D}, r}\left(p^{(n+1)}-q^{(n+1)}\right) .
\end{aligned}
$$

5 By gathering the results and using the fact that $\tilde{T}_{p, 4}=a_{\mathcal{T}, \lambda_{\mathrm{T}}, u_{1}^{(n+1)}}\left(p^{(n+1)}, q^{(n+1)}, p^{(n+1)}-q^{(n+1)}\right)$, we obtain

$$
\begin{aligned}
\hat{C}_{1}\left\|p^{(n+1)}-q^{(n+1)}\right\|_{\mathcal{T}}^{2} \leq & \bar{C}_{1 m_{0}}\left\|m_{0}\left(u_{1}^{(n+1)}, u_{0}^{(n+1)}\right)-m_{0}\left(u^{(n+1)}, u^{(n+1)}\right)\right\|_{L^{2}(\Omega)}\left\|p^{(n+1)}-q^{(n+1)}\right\|_{\mathcal{T}} \\
& +\int_{\Omega}\left(\lambda_{\mathrm{T}} \varrho_{f}\right)\left(u^{(n+1)}\right) \Lambda \mathrm{g} \cdot \widetilde{\nabla}_{\mathcal{D}, \Upsilon}\left(p^{(n+1)}-q^{(n+1)}\right) \\
& +\int_{\Omega}\left(s^{+}-s^{-}\right)\left(p^{(n+1)}-q^{(n+1)}\right) \\
& -a_{\mathcal{T}, \lambda_{\mathrm{T}}, u_{1}^{(n+1)}}\left(p^{(n+1)}, q^{(n+1)}, p^{(n+1)}-q^{(n+1)}\right) .
\end{aligned}
$$


After setting

$\tilde{T}_{u, 6}=-\sum_{K \in \mathcal{T}} \sum_{\sigma \in \mathcal{E}_{K}} F_{K, \sigma}\left(\psi\left(u^{(n+1)}\right), v^{(n+1)}\right)\left(\psi\left(u_{K}^{(n+1)}\right)-v_{K}^{(n+1)}\right)=a_{\mathcal{T}, 1,1}\left(\psi\left(u^{(n+1)}\right), v^{(n+1)}, \psi\left(u^{(n+1)}\right)-v^{(n+1)}\right)$,

First using Young's inequality, then multiplying the obtained inequality by $\delta t^{\left(n+\frac{1}{2}\right)}$ and summing it up over $n=0, \ldots, N-1$, yield

$$
\begin{aligned}
\|p-q\|_{\mathscr{T}}^{2} \leq \frac{4}{3 \hat{C}_{1}}( & \frac{\bar{C}_{1}^{2} m_{0}}{\hat{C}_{1}}\left\|m_{0}\left(u_{1}, u_{0}\right)-m_{0}(u, u)\right\|_{L^{2}(\Omega \times(0, T))}^{2} \\
& +\int_{0}^{T} \int_{\Omega}\left(\lambda_{\mathrm{T}} \varrho_{f}\right)(u) \Lambda \mathrm{g} \cdot \widetilde{\nabla}_{\mathcal{D}, \Upsilon}(p-q) \\
& +\int_{0}^{T} \int_{\Omega}\left(s^{+}-s^{-}\right)(p-q) \\
& \left.-\sum_{n=0}^{N-1} \delta t^{\left(n+\frac{1}{2}\right)} a_{\mathcal{T}, \lambda_{\mathrm{T}}, u_{1}^{(n+1)}}\left(p^{(n+1)}, q^{(n+1)}, p^{(n+1)}-q^{(n+1)}\right)\right) .
\end{aligned}
$$

Thanks to the coercivity property (34),

$$
T_{u, 1} \geq \hat{C}_{1}\left\|\psi\left(u^{(n+1)}\right)-v^{(n+1)}\right\|_{\mathcal{T}}^{2} .
$$

Multiplying equation 28a by $\psi\left(u_{K}^{(n+1)}\right)-v_{K}^{(n+1)}$ and summing it over $K \in \mathcal{T}$ results in $\tilde{T}_{u, 1}=$ $\tilde{T}_{u, 2}+\tilde{T}_{u, 3}+\tilde{T}_{u, 4}+\tilde{T}_{u, 5}$, with

$$
\begin{aligned}
\tilde{T}_{u, 1}= & -\sum_{K \in \mathcal{T}} \sum_{\sigma \in \mathcal{E}_{K}} F_{K, \sigma}\left(\psi\left(u^{(n+1)}\right), \psi\left(u^{(n+1)}\right)\right)\left(\psi\left(u_{K}^{(n+1)}\right)-v_{K}^{(n+1)}\right), \\
\tilde{T}_{u, 2}= & -\sum_{K \in \mathcal{T}}|K| \frac{u_{K}^{(n+1)}-u_{K}^{(n)}}{\delta t^{\left(n+\frac{1}{2}\right)}}\left(\psi\left(u_{K}^{(n+1)}\right)-v_{K}^{(n+1)}\right), \\
\tilde{T}_{u, 3}= & -\sum_{K \in \mathcal{T}} \sum_{\sigma \in \mathcal{E}_{K}} f_{1}\left(u_{1, \sigma}^{(n+1)}\right) \mathbf{v}_{K, \sigma}^{(n+1)}\left(\psi\left(u_{K}^{(n+1)}\right)-v_{K}^{(n+1)}\right), \\
\tilde{T}_{u, 4}= & \sum_{K \in \mathcal{T}} \sum_{\sigma \in \mathcal{E}_{K}} f_{1}\left(u_{2, \sigma}^{(n+1)}\right) \lambda_{2}\left(u_{3, \sigma}^{(n+1)}\right)\left(\varrho_{2}-\varrho_{1}\right) G_{K, \sigma}\left(\psi\left(u_{K}^{(n+1)}\right)-v_{K}^{(n+1)}\right), \\
\tilde{T}_{u, 5}= & \sum_{K \in \mathcal{T}}|K|\left(f_{1}\left(c_{K}\right) s_{K}^{+}-f_{1}\left(u_{K}^{(n+1)}\right) s_{K}^{-}\right)\left(\psi\left(u_{K}^{(n+1)}\right)-v_{K}^{(n+1)}\right) .
\end{aligned}
$$

we observe that $T_{u, 1}=\tilde{T}_{u, 2}+\tilde{T}_{u, 3}+\tilde{T}_{u, 4}+\tilde{T}_{u, 5}-\tilde{T}_{u, 6}$. From inequality (14), we deduce that

$$
\tilde{T}_{u, 2} \leq-\sum_{K \in \mathcal{T}}|K| \frac{\Psi\left(u_{K}^{(n+1)}\right)-\Psi\left(u_{K}^{(n)}\right)}{\delta t^{\left(n+\frac{1}{2}\right)}}+\int_{\Omega}\left(\delta_{t} u\right)^{(n)} v^{(n+1)} .
$$

404

By using the expression of $\mathbf{v}_{K, \sigma}^{(n+1)}$, we observe that

$$
\begin{aligned}
\tilde{T}_{u, 3}= & -a_{\mathcal{T}, \lambda_{\mathrm{T}} f_{1}, u_{1}^{(n+1)}}\left(p^{(n+1)}, p^{(n+1)}, \psi\left(u^{(n+1)}\right)-v^{(n+1)}\right) \\
& -\sum_{K \in \mathcal{T}} \sum_{\sigma \in \mathcal{E}_{K}}\left(f_{1} \lambda_{\mathrm{T}}\right)\left(u_{1, \sigma}^{(n+1)}\right) \varrho_{f}\left(u_{0, \sigma}^{(n+1)}\right) G_{K, \sigma}\left(\psi\left(u_{K}^{(n+1)}\right)-v_{K}^{(n+1)}\right) .
\end{aligned}
$$


405

Thanks again to Lemma 7, we have

$$
\begin{aligned}
\tilde{T}_{u, 4} \leq & \bar{C}_{1 m_{2}}\left\|m_{2}\left(u_{2}^{(n+1)}, u_{3}^{(n+1)}\right)-m_{2}\left(u^{(n+1)}, u^{(n+1)}\right)\right\|_{L^{2}(\Omega)}\left\|\psi\left(u^{(n+1)}\right)-v^{(n+1)}\right\|_{\mathcal{T}} \\
& +\int_{\Omega}\left(f_{1} \lambda_{2}\right)\left(u^{(n+1)}\right)\left(\varrho_{1}-\varrho_{2}\right) \Lambda \mathbf{g} \cdot \widetilde{\nabla}_{\mathcal{D}, \Upsilon}\left(\psi\left(u^{(n+1)}\right)-v^{(n+1)}\right) .
\end{aligned}
$$

409

$$
\begin{aligned}
& \hat{C}_{1}\left\|\psi\left(u^{(n+1)}\right)-v^{(n+1)}\right\|_{\mathcal{T}}^{2} \leq-\sum_{K \in \mathcal{T}}|K| \frac{\Psi\left(u_{K}^{(n+1)}\right)-\Psi\left(u_{K}^{(n)}\right)}{\delta t^{\left(n+\frac{1}{2}\right)}}+\int_{\Omega}\left(\delta_{t} u\right)^{(n)} v^{(n+1)} \\
& +\bar{C}_{1 m_{1}}\left\|m_{1}\left(u_{1}^{(n+1)}, u_{0}^{(n+1)}\right)-m_{1}\left(u^{(n+1)} u^{(n+1)}\right)\right\|_{L^{2}(\Omega)}\left\|\psi\left(u^{(n+1)}\right)-v^{(n+1)}\right\|_{\mathcal{T}} \\
& \left.+\bar{C}_{1 m_{2}}\left\|m_{2}\left(u_{2}^{(n+1)}, u_{3}^{(n+1)}\right)-m_{2}\left(u^{(n+1)}, u^{(n+1)}\right)\right\|_{L^{2}(\Omega)}\right)\left\|\psi\left(u^{(n+1)}\right)-v^{(n+1)}\right\|_{\mathcal{T}} \\
& +\int_{\Omega}\left(f_{1} \lambda_{\mathrm{T}} \varrho_{f}\right)\left(u^{(n+1)}\right) \Lambda \mathbf{g} \cdot \widetilde{\nabla}_{\mathcal{D}, Y}\left(\psi\left(u^{(n+1)}\right)-v^{(n+1)}\right) \\
& +C_{\chi}\left\|p^{(n+1)}-q^{(n+1)}\right\|_{\mathcal{T}} \| \psi\left(u^{(n+1)}\right)-\psi\left(v^{(n+1)} \|_{\mathcal{T}}\right. \\
& -a_{\mathcal{T}, \lambda_{\mathrm{T}} f_{1}, u_{1}^{(n+1)}}\left(p^{(n+1)}, q^{(n+1)}, \psi\left(u^{(n+1)}\right)-v^{(n+1)}\right) \\
& +\int_{\Omega}\left(f_{1} \lambda_{2}\right)\left(u^{(n+1)}\right)\left(\varrho_{1}-\varrho_{2}\right) \Lambda \mathbf{g} \cdot \widetilde{\nabla}_{\mathcal{D}, \Upsilon}\left(\psi\left(u^{(n+1)}\right)-v^{(n+1)}\right) \\
& +\int_{\Omega}\left(f_{1}(c) s^{+}-f_{1}\left(u^{(n+1)}\right) s^{-}\right)\left(\psi\left(u^{(n+1)}\right)-v^{(n+1)}\right) \\
& -a_{\mathcal{T}, 1,1}\left(\psi\left(u^{(n+1)}\right), v^{(n+1)}, \psi\left(u^{(n+1)}\right)-v^{(n+1)}\right) .
\end{aligned}
$$




$$
\begin{aligned}
& \int_{0}^{T}\|\psi(u)-v\|_{\mathcal{T}}^{2} \leq \frac{4}{\hat{C}_{1}}\left(-\left(\left\|\Psi\left(u^{(N)}\right)\right\|_{L^{1}(\Omega)}-\left\|\Psi\left(u^{0}\right)\right\|_{L^{1}(\Omega)}\right)+\int_{0}^{T} \int_{\Omega} \delta_{t} u v\right. \\
& +\frac{\bar{C}_{1 m_{1}}^{2}}{\hat{C}_{1}}\left\|m_{1}\left(u_{1}, u_{0}\right)-m_{1}(u, u)\right\|_{L^{2}(\Omega) \times(0, T)}^{2} \\
& +\frac{\bar{C}_{1 m_{2}}^{2}}{\hat{C}_{1}}\left\|m_{2}\left(u_{2}, u_{3}\right)-m_{2}(u, u)\right\|_{L^{2}(\Omega \times(0, T))}^{2} \\
& +\frac{C_{\chi}^{2}}{\hat{C}_{1}} \int_{0}^{T}\|p-q\|_{\mathcal{T}}^{2} \\
& -\sum_{n=0}^{N-1} \delta t^{\left(n+\frac{1}{2}\right)} a_{\mathcal{T}, \lambda_{\mathrm{T}} f_{1}, u_{1}^{(n+1)}}\left(p^{(n+1)}, q^{(n+1)}, \psi\left(u^{(n+1)}\right)-v^{(n+1)}\right) \\
& +\int_{0}^{T} \int_{\Omega}\left(f_{1} \lambda_{\mathrm{T}} \varrho_{f}+f_{1} \lambda_{2}\left(\varrho_{1}-\varrho_{2}\right)\right)(u) \Lambda \mathbf{g} \cdot \widetilde{\nabla}_{\mathcal{D}, \Upsilon}(\psi(u)-v) \\
& +\int_{0}^{T} \int_{\Omega}\left(f_{1}(c) s^{+}-f_{1}(u) s^{-}\right)(\psi(u)-v) \\
& \left.-\sum_{n=0}^{N-1} \delta t^{\left(n+\frac{1}{2}\right)} a_{\mathcal{T}, 1,1}\left(\psi\left(u^{(n+1)}\right), v^{(n+1)}, \psi\left(u^{(n+1)}\right)-v^{(n+1)}\right)\right) .
\end{aligned}
$$

Lemma 9 (Strong convergence) Let $\mathscr{D}_{m}$ be a sequence of space-time discretizations matching Definition 4 and $\left(p_{m}, u_{m}\right) \in\left[H_{\mathscr{T}}(\Omega \times(0, T))\right]^{2}$ be a discrete solution of problem (28). Let us assume that Hypotheses 1 and 2 hold. Then, there exist $\psi(\bar{u}), \bar{p} \in L^{2}\left(0, T ; H_{0}^{1}(\Omega)\right)$ such that

$$
\begin{aligned}
& \lim _{m \rightarrow \infty}\left\|p_{m}-\bar{p}_{\mathscr{T}_{m}}\right\|_{\mathscr{T}_{m}}=0, \\
& \lim _{m \rightarrow \infty}\left\|\psi\left(u_{m}\right)-\psi(\bar{u})_{\mathscr{T}_{m}}\right\|_{\mathscr{T}_{m}}=0
\end{aligned}
$$

where, for any $m \in \mathbb{N}$ and $v \in L^{2}(\Omega \times(0, T)), v_{\mathscr{T}_{m}}$ is defined in Section 3.2.2.

Proof Let $\psi(\bar{u}), \bar{p} \in L^{2}\left(0, T ; H_{0}^{1}(\Omega)\right)$ be the functions given from Lemma 6 and let $\varphi, \chi \in L^{2}(0, T ; \mathfrak{D})$. For any $m \in \mathbb{N}$, we have

$$
\begin{array}{r}
\left\|p_{m}-\bar{p}_{\mathscr{T}_{m}}\right\| \mathscr{T}_{m} \leq\left\|p_{m}-\varphi_{\mathscr{T}_{m}}\right\|_{\mathscr{T}_{m}}+\left\|\varphi_{\mathscr{T}_{m}}-\bar{p}_{\mathscr{T}_{m}}\right\|_{\mathscr{T}_{m}} \\
\left\|\psi\left(u_{m}\right)-\psi(\bar{u})_{\mathscr{T}_{m}}\right\| \mathscr{T}_{m} \leq\left\|\psi\left(u_{m}\right)-\chi \mathscr{T}_{m}\right\|_{\mathscr{T}_{m}}+\left\|\chi \mathscr{T}_{m}-\psi(\bar{u})_{\mathscr{T}_{m}}\right\|_{\mathscr{T}_{m}}
\end{array}
$$

$\left\|\varphi_{\mathscr{T}_{m}}-\bar{p}_{\mathscr{T}_{m}}\right\|_{\mathscr{T}_{m}} \leq C_{17}\|\varphi-\bar{p}\|_{L^{2}\left(0, T ; H_{0}^{1}(\Omega)\right)}$,

$\left\|\chi \mathscr{T}_{m}-\psi(\bar{u})_{\mathscr{T}_{m}}\right\|_{\mathscr{T}_{m}} \leq C_{17}\|\chi-\psi(\bar{u})\|_{L^{2}\left(0, T ; H_{0}^{1}(\Omega)\right)}$. 
Thanks to Lemma 8, (i) (viii) of Lemma 6, (35) and the properties of the functions $\lambda_{\mathrm{T}}, \lambda_{1}, \lambda_{2}$, we infer that

$$
\begin{aligned}
& \limsup _{m \rightarrow \infty}\left\|p_{m}-\bar{p} \mathscr{T}_{m}\right\| \mathscr{T}_{m} \quad \leq \bar{C}_{2}\left(\int_{0}^{T} \int_{\Omega} \lambda_{\mathrm{T}} \varrho_{f}(\bar{u}) \Lambda \mathbf{g} \cdot \nabla(\bar{p}-\varphi)\right. \\
& +\int_{0}^{T^{T}} \int_{\Omega}\left(s^{+}-s^{-}\right)(\bar{p}-\varphi) \\
& \left.-\int_{0}^{T} \int_{\Omega} \lambda_{\mathrm{T}}(\bar{u}) \Lambda \nabla \varphi \cdot \nabla(\bar{p}-\varphi)\right) \\
& +C_{17}\|\varphi-\bar{p}\|_{L^{2}\left(0, T ; H_{0}^{1}(\Omega)\right)}, \\
& \limsup _{m \rightarrow \infty}\left\|\psi\left(u_{m}\right)-\psi(\bar{u})_{\mathscr{T}_{m}}\right\|_{\mathscr{T}_{m}} \leq \bar{C}_{4}\left(-\left(\|\Psi(\bar{u}(T))\|_{L^{1}(\Omega)}-\|\Psi(\bar{u}(0))\|_{L^{1}(\Omega)}\right)+\int_{0}^{T} \int_{\Omega} \partial_{t} \bar{u} \psi(\bar{u})\right. \\
& +\bar{C}_{7} \limsup \left\|p_{m}-\bar{p} \mathscr{T}_{m}\right\| \mathscr{T}_{m} \\
& -\int_{0}^{T} \int_{\Omega}^{m \rightarrow \infty}\left(\lambda_{\mathrm{T}} f_{1}\right)(\bar{u}) \Lambda \nabla \varphi \cdot \nabla(\psi(\bar{u})-\chi) \\
& +\int_{0}^{T} \int_{\Omega}\left(f_{1} \lambda_{\mathrm{T}} \varrho_{f}+f_{1} \lambda_{2}\left(\varrho_{1}-\varrho_{2}\right)\right)(u) \Lambda \mathbf{g} \cdot \nabla(\psi(\bar{u})-\chi) \\
& +\int_{0}^{T} \int_{\Omega}\left(f_{1}(c) s^{+}-f_{1}(u) s^{-}\right)(\psi(\bar{u})-\chi)- \\
& \left.\int_{0}^{T} \int_{\Omega} \Lambda \nabla \chi \cdot \nabla(\psi(\bar{u})-\chi)\right) \\
& +C_{17}\|\chi-\psi(\bar{u})\|_{L^{2}\left(0, T ; H_{0}^{1}(\Omega)\right)}
\end{aligned}
$$

Since $L^{2}(0, T ; \mathfrak{D})$ is dense in $L^{2}\left(0, T ; H_{0}^{1}(\Omega)\right)$, by letting $\varphi \rightarrow \bar{p}$ in $L^{2}\left(0, T ; H_{0}^{1}(\Omega)\right)$ and $\chi \rightarrow \psi(\bar{u})$, the previous inequality leads to

$$
\begin{aligned}
& \limsup _{m \rightarrow \infty}\left\|p_{m}-\bar{p}_{\mathscr{T}_{m}}\right\| \mathscr{T}_{m}=0, \\
& \limsup _{m \rightarrow \infty}\left\|\psi\left(u_{m}\right)-\psi(\bar{u})_{\mathscr{T}_{m}}\right\|_{\mathscr{T}_{m}}=0 .
\end{aligned}
$$

The convergence of the discrete solutions to some pair $(\bar{u}, \bar{p})$ has already been shown in Lemma 6 and 9 it remains to show that $(\bar{u}, \bar{p})$ is indeed a weak solution. This is done in the following theorem.

Theorem 1 (Convergence of the scheme) Let $\mathscr{D}_{m}$ be a sequence of space-time discretizations matching Definition 4 and $\left(p_{m}, u_{m}\right) \in\left[H_{\mathscr{T}}(\Omega \times(0, T))\right]^{2}$ be a discrete solution of problem (28). Assume that Hypotheses 1 and 2 hold. Then $\left(p_{m}, u_{m}\right)$ converges, as $m \rightarrow \infty$, to the pair $(\bar{p}, \bar{u})$ (according to Lemma 6 and 9), and this pair is a weak solution of problem (15).

Proof Let $\varphi \in L^{2}(0, T ; \mathfrak{D})$. For any $m \in \mathbb{N}, n \in \llbracket 0, N_{m} \rrbracket$, we multiply equation $28 \mathrm{~b}$ by $\left(\varphi \mathscr{T}_{m}\right)_{K}^{(n+1)}$ and sum it over $K \in \mathcal{T}_{m}$ and $n \in \llbracket 0, N_{m} \rrbracket$ to obtain

$$
\sum_{n=0}^{N_{m}} \delta t^{(n+1)}\left(\tilde{T}_{p, 1}^{(n+1)}-\tilde{T}_{p, 2}^{(n+1)}-\tilde{T}_{p, 3}^{(n+1)}\right)=0
$$

432 with

$$
\begin{aligned}
\tilde{T}_{p, 1}^{(n+1)} & =-\sum_{K \in \mathcal{T}_{m}} \sum_{\sigma \in \mathcal{E}_{K}} \lambda_{\mathrm{T}}\left(\left(u_{m}\right)_{1, \sigma}^{(n+1)}\right) F_{K, \sigma}\left(p_{m}^{(n+1)}, p_{m}^{(n+1)}\right)\left(\varphi_{\mathscr{T}_{m}}\right)_{K}^{(n+1)} \\
& =a_{\mathcal{T}_{m}, \lambda_{\mathrm{T}},\left(u_{m}\right)_{1}^{(n+1)}\left(p_{m}^{(n+1)}, p_{m}^{(n+1)}, \varphi_{\mathscr{T}_{m}}^{(n+1)}\right),} \\
\tilde{T}_{p, 2}^{(n+1)} & =-\sum_{K \in \mathcal{T}_{m}} \sum_{\sigma \in \mathcal{E}_{K}} \lambda_{\mathrm{T}}\left(\left(u_{m}\right)_{1, \sigma}^{(n+1)}\right) \varrho_{f}\left(\left(u_{m}\right)_{0, \sigma}^{(n+1)}\right) G_{K, \sigma}\left(\varphi_{\mathscr{T}_{m}}\right)_{K}^{(n+1)}, \\
\tilde{T}_{p, 3}^{(n+1)} & =\sum_{K \in \mathcal{T}_{m}}|K|\left(s_{K}^{+}-s_{K}^{-}\right)\left(\varphi_{\mathscr{T}_{m}}\right)_{K}^{(n+1)}=\int_{\Omega}\left(s^{+}-s^{-}\right) \varphi_{\mathscr{T}_{m}}^{(n+1)} .
\end{aligned}
$$


Thanks to the properties (P1), (P2), the Cauchy-Schwarz inequality and Lemma 11, we deduce

$$
\begin{aligned}
\left|\int_{0}^{T} a_{\mathcal{T}_{m}, \lambda_{\mathrm{T}},\left(u_{m}\right)_{1}(t)}\left(p_{m}(t), p_{m}(t)-\bar{p}_{\mathscr{T}_{m}}(t), \varphi \mathscr{T}_{m}(t)\right)\right| & \leq C_{\chi}\left\|p_{m}-\bar{p}_{\mathscr{T}_{m}}\right\| \mathscr{T}_{m}\left\|\varphi_{\mathscr{T}_{m}}\right\|_{\mathscr{T}_{m}} \\
& \leq C_{17} C_{\chi}\left\|p_{m}-\bar{p}_{\mathscr{T}_{m}}\right\| \mathscr{T}_{m}\|\varphi\|_{L^{2}\left(0, T ; H_{0}^{1}(\Omega)\right)} .
\end{aligned}
$$

Similarly, by using the same ideas as for 59 and by means of the Cauchy-Schwarz inequality and Lemma 11, we get

$$
\begin{aligned}
\mid \sum_{n=0}^{N_{m}} \delta t^{(n+1)} \tilde{T}_{p, 2}^{(n+1)} & -\int_{0}^{T} \int_{\Omega}\left(\lambda_{\mathrm{T}} \varrho_{f}\right)\left(u_{m}\right) \Lambda \mathbf{g} \cdot \widetilde{\nabla}_{\mathcal{D}_{m}, \Upsilon_{m}} \varphi_{\mathscr{T}_{m}} \mid \\
& \leq C_{17} \bar{C}_{1 m_{0}}\left\|m_{0}\left(\left(u_{m}\right)_{1},\left(u_{m}\right)_{0}\right)-m_{0}\left(u_{m}, u_{m}\right)\right\|_{L^{2}((0, T) \times \Omega)}\|\varphi\|_{L^{2}\left(0, T ; H_{0}^{1}(\Omega)\right)},
\end{aligned}
$$

with $m_{0}(x, y)=\lambda_{\mathrm{T}}(x) \varrho_{f}(y)$. Thanks to $\left.65-67\right)$, we obtain

$$
\begin{aligned}
& \left|\int_{0}^{T} a_{\mathcal{T}_{m}, \lambda_{\mathrm{T}},\left(u_{m}\right)_{1}(t)}\left(p_{m}(t), \bar{p}_{\mathscr{T}_{m}}(t), \varphi_{\mathscr{T}_{m}}(t)\right)-\int_{0}^{T} \int_{\Omega}\left(s^{+}-s^{-}\right) \varphi_{\mathscr{T}_{m}}-\int_{0}^{T} \int_{\Omega}\left(\lambda_{\mathrm{T}} \varrho_{f}\right)\left(u_{m}\right) \Lambda \mathbf{g} \cdot \widetilde{\nabla}_{\mathcal{D}_{m}, Y_{m}} \varphi_{\mathscr{T}_{m}}\right| \\
& \leq C_{17} \max \left(C_{\chi}, \bar{C}_{1 m_{0}}\right)\left(\begin{array}{l}
\left\|p_{m}-\bar{p}_{\mathscr{T}_{m}}\right\| \mathscr{T}_{m}+ \\
\left\|m_{0}\left(\left(u_{m}\right)_{1},\left(u_{m}\right)_{2}\right)-m_{0}\left(u_{m}, u_{m}\right)\right\|_{L^{2}((0, T) \times \Omega)}
\end{array}\right)\|\varphi\|_{L^{2}\left(0, T ; H_{0}^{1}(\Omega)\right)} .
\end{aligned}
$$

By using the Lemma 6 and 9 and Proposition 4 , as $m \rightarrow \infty$, we obtain

$$
\int_{0}^{T} \int_{\Omega} \lambda_{\mathrm{T}}(\bar{u}) \Lambda \nabla \bar{p} \cdot \nabla \varphi-\int_{0}^{T} \int_{\Omega}\left(s^{+}-s^{-}\right) \varphi-\int_{0}^{T} \int_{\Omega}\left(\lambda_{\mathrm{T}} \varrho_{f}\right)(\bar{u}) \Lambda \mathbf{g} \cdot \nabla \varphi=0 .
$$

For any $m \in \mathbb{N}, n \in \llbracket 0, N_{m} \rrbracket$, we multiply equation $(28 \mathrm{a})$ by $\left(\varphi_{\mathscr{T}_{m}}\right)_{K}^{(n+1)}$ and sum it over $K \in \mathcal{T}_{m}$ and $n \in \llbracket 0, N_{m} \rrbracket$ to obtain

$$
\sum_{n=0}^{N_{m}} \delta t^{(n+1)}\left(\tilde{T}_{u, 1}^{(n+1)}-\tilde{T}_{u, 2}^{(n+1)}-\tilde{T}_{u, 3}^{(n+1)}-\tilde{T}_{u, 4}^{(n+1)}-\tilde{T}_{u, 5}^{(n+1)}\right)=0,
$$

9

with

$$
\begin{aligned}
\tilde{T}_{u, 1}^{(n+1)} & =-\sum_{K \in \mathcal{T}_{m}} \sum_{\sigma \in \mathcal{E}_{K}} F_{K, \sigma}\left(\psi\left(u_{m}^{(n+1)}\right), \psi\left(u_{m}^{(n+1)}\right)\right)\left(\varphi_{\mathscr{T}_{m}}\right)_{K}^{(n+1)}=a_{\mathcal{T}_{m}, 1,1}\left(\psi\left(u_{m}^{(n+1)}\right), \psi\left(u_{m}^{(n+1)}\right), \varphi_{\mathscr{T}_{m}}^{(n+1)}\right) \\
\tilde{T}_{u, 2}^{(n+1)} & =-\sum_{K \in \mathcal{T}_{m}}|K| \frac{\left(u_{m}\right)_{K}^{(n+1)}-\left(u_{m}\right)_{K}^{(n)}}{\delta t^{\left(n+\frac{1}{2}\right)}}\left(\varphi \mathscr{T}_{m}\right)_{K}^{(n+1)}=-\int_{\Omega}\left(\delta_{t} u_{m}\right)^{(n+1)} \varphi_{\mathscr{T}_{m}}^{(n+1)} \\
\tilde{T}_{u, 3}^{(n+1)} & =-\sum_{K \in \mathcal{T}_{m}} \sum_{\sigma \in \mathcal{E}_{K}} f_{1}\left(\left(u_{m}\right)_{1, \sigma}^{(n+1)}\right) \mathbf{v}_{K, \sigma}^{(n+1)}\left(\varphi \mathscr{T}_{m}\right)_{K}^{(n+1)}, \\
\tilde{T}_{u, 4}^{(n+1)} & =\sum_{K \in \mathcal{T}_{m}} \sum_{\sigma \in \mathcal{E}_{K}} f_{1}\left(\left(u_{m}\right)_{2, \sigma}^{(n+1)}\right) \lambda_{2}\left(\left(u_{m}\right)_{3, \sigma}^{(n+1)}\right)\left(\varrho_{2}-\varrho_{1}\right) G_{K, \sigma}\left(\varphi_{\mathscr{T}_{m}}\right)_{K}^{(n+1)} \\
\tilde{T}_{u, 5}^{(n+1)} & =\sum_{K \in \mathcal{T}_{m}}|K|\left(f_{1}\left(c_{K}\right) s_{K}^{+}-f_{1}\left(\left(u_{m}\right)_{K}^{(n+1)}\right) s_{K}^{-}\right)\left(\varphi_{\mathscr{T}_{m}}\right)_{K}^{(n+1)}=\int_{\Omega}\left(f_{1}(c) s^{+}-f_{1}\left(u_{m}^{(n+1)}\right) s^{-}\right)\left(\varphi_{\mathscr{T}_{m}}\right)^{(n+1)}
\end{aligned}
$$

In the same way as for $(66)$ and $(67)$, we can prove that

$$
\begin{aligned}
\mid \sum_{n=0}^{N_{m}} \delta t^{(n+1)} \tilde{T}_{u, 1}^{(n+1)}-\int_{0}^{T} a_{\mathcal{T}_{m}, 1,1}( & \left.\psi\left(u_{m}(t)\right), \psi(\bar{u})_{\mathscr{T}_{m}}(t), \varphi_{\mathscr{T}_{m}}(t)\right) \mid \\
\leq & C_{17} C_{\chi}\left\|\psi\left(u_{m}\right)-\psi(\bar{u})_{\mathscr{T}_{m}}\right\| \mathscr{T}_{m}\|\varphi\|_{L^{2}\left(0, T ; H_{0}^{1}(\Omega)\right)},
\end{aligned}
$$


441

$$
\begin{aligned}
& \left|\sum_{n=0}^{N_{m}} \delta t^{(n+1)} \tilde{T}_{u, 3}^{(n+1)}+\int_{0}^{T} \int_{\Omega}\left(f_{1} \lambda_{\mathrm{T}} \varrho_{f}\right)\left(u_{m}\right) \Lambda \mathbf{g} \cdot \widetilde{\nabla}_{\mathcal{D}_{m}, \Upsilon_{m}} \varphi_{\mathscr{T}_{m}}+\int_{0}^{T} a_{\mathcal{T}_{m}, \lambda_{\mathrm{T}} f_{1},\left(u_{m}\right)_{1}(t)}\left(p_{m}(t), \bar{p} \bar{T}_{m}(t), \varphi_{\mathscr{T}_{m}}(t)\right)\right| \\
& \leq C_{17} \max \left(C_{\chi}, \bar{C}_{1 m_{1}}\right)\left(\left\|m_{1}\left(\left(u_{m}\right)_{1},\left(u_{m}\right)_{0}\right)-m_{1}\left(u_{m}, u_{m}\right)\right\|_{L^{2}((0, T) \times \Omega)}+\left\|p_{m}-\bar{p}_{\mathscr{T}_{m}}\right\| \mathscr{T}_{m}\right)\|\varphi\|_{L^{2}\left(0, T ; H_{0}^{1}(\Omega)\right)}
\end{aligned}
$$

with $m_{1}(x, y)=\left(f_{1} \lambda_{\mathrm{T}}\right)(x) \varrho_{f}(y)$ and

$$
\begin{aligned}
& \left|\sum_{n=0}^{N_{m}} \delta t^{(n+1)} \tilde{T}_{u, 4}^{(n+1)}+\int_{0}^{T} \int_{\Omega}\left(f_{1} \lambda_{2}\right)\left(u_{m}\right)\left(\varrho_{2}-\varrho_{1}\right) \Lambda \mathbf{g} \cdot \widetilde{\nabla}_{\mathcal{D}_{m}, \Upsilon_{m}} \varphi \mathscr{T}_{m}\right| \leq C_{17} \bar{C}_{1 m_{2}}\left\|m_{2}\left(\left(u_{m}\right)_{2},\left(u_{m}\right)_{3}\right)-m_{2}\left(u_{m}, u_{m}\right)\right\|_{L^{2}((0, T) \times \Omega)}\|\varphi\|_{L^{2}\left(0, T ; H_{0}^{1}(\Omega)\right)}
\end{aligned}
$$

with $m_{2}(x, y)=f_{1}(x) \lambda_{2}(y)\left(\varrho_{2}-\varrho_{1}\right)$. Thanks to $68-(71)$, we obtain

$$
\begin{aligned}
& \left|\begin{array}{l}
\int_{0}^{T} a_{\mathcal{T}_{m}, 1,1}\left(\psi\left(u_{m}(t)\right), \psi(\bar{u})_{\mathscr{T}_{m}}(t), \varphi_{\mathscr{T}_{m}}(t)\right)+\int_{0}^{T} \int_{\Omega} \delta_{t} u_{m} \varphi_{\mathscr{T}_{m}} \\
+\int_{0}^{T} \int_{\Omega}\left(f_{1} \lambda_{\mathrm{T}} \varrho_{f}\right)\left(u_{m}\right) \Lambda \mathbf{g} \cdot \widetilde{\nabla}_{\mathcal{D}_{m}, \Upsilon_{m}} \varphi_{\mathscr{T}_{m}}+\int_{0}^{T} a_{\mathcal{T}_{m}, \lambda_{\mathrm{T}} f_{1},\left(u_{m}\right)_{1}(t)}\left(p_{m}(t), \bar{p}_{\mathscr{T}_{m}}(t), \varphi_{\mathscr{T}_{m}}(t)\right) \\
+\int_{0}^{T} \int_{\Omega}\left(f_{1} \lambda_{2}\right)\left(u_{m}\right)\left(\varrho_{1}-\varrho_{2}\right) \Lambda \mathbf{g} \cdot \widetilde{\nabla}_{\mathcal{D}_{m}, \Upsilon_{m}} \varphi_{\mathscr{T}_{m}}-\int_{0}^{T} \int_{\Omega}\left(f_{1}(c) s^{+}-f_{1}\left(u_{m}\right) s^{-}\right) \varphi_{\mathscr{T}_{m}}
\end{array}\right| \\
& \leq C_{17} \max \left(C_{\chi}, \bar{C}_{1 m_{1}}, \bar{C}_{1 m_{2}}\right)\left(\begin{array}{l}
\left\|\psi\left(u_{m}\right)-\psi(\bar{u})_{\mathscr{T}_{m}}\right\| \mathscr{T}_{m} \\
+\left\|m_{1}\left(\left(u_{m}\right)_{1},\left(u_{m}\right)_{0}\right)-m_{1}\left(u_{m}, u_{m}\right)\right\|_{L^{2}((0, T) \times \Omega)} \\
+\left\|p_{m}-\bar{p}_{\mathscr{T}_{m}}\right\|_{\mathscr{T}_{m}} \\
+\left\|m_{2}\left(\left(u_{m}\right)_{2},\left(u_{m}\right)_{3}\right)-m_{2}\left(u_{m}, u_{m}\right)\right\|_{L^{2}((0, T) \times \Omega)}
\end{array}\right)\|\varphi\|_{L^{2}\left(0, T ; H_{0}^{1}(\Omega)\right)} .
\end{aligned}
$$

By using Lemma 6 and 9 and Proposition 4, as $m \rightarrow \infty$, we therefore obtain

$$
\begin{aligned}
& \int_{0}^{T} \int_{\Omega}-\bar{u} \varphi_{t}-\int_{\Omega} u_{\text {init }}(\mathbf{x}) \varphi(\mathbf{x}, 0)+ \\
& \int_{0}^{T} \int_{\Omega}\left(\lambda_{\mathrm{T}} f_{1}\right)(\bar{u}) \Lambda \nabla \bar{p} \cdot \nabla \varphi-\int_{0}^{T} \int_{\Omega}\left(f_{1} \lambda_{\mathrm{T}} \varrho_{f}\right)(\bar{u}) \Lambda \mathbf{g} \cdot \nabla \varphi- \\
& \int_{0}^{T} \int_{\Omega}^{T}\left(f_{1} \lambda_{2}\right)(\bar{u})\left(\varrho_{1}-\varrho_{2}\right) \Lambda \mathbf{g} \cdot \nabla \varphi+ \\
& \int_{0}^{T} \int_{\Omega}^{T} \Lambda \nabla \psi(\bar{u}) \cdot \nabla \varphi- \\
& \int_{0}^{T} \int_{\Omega}\left(f_{1}(c) s^{+}-f_{1}(\bar{u}) s^{-}\right) \varphi=0 .
\end{aligned}
$$

\section{Flux discretizations}

In this section, we introduce a specific familiy of discrete diffusive fluxes $F_{K, \sigma}$, which are used in the equations (28). Hereby, we mainly follow the ideas that have been presented in [34,36]. Please note that the fluxes do not include the phase mobilities which are evaluated separately such that the fluxes can be constructed analogously.

Please note that in the following, we will define the fluxes based on some $u, v \in H_{\mathcal{T}}(\Omega)$, where $u$ is not meant to be the saturation. In the discrete formulation (28), the fluxes are then evaluated for $u=p^{(n+1)}$ or $u=\psi\left(u^{(n+1)}\right)$. An established idea to obtain monotone or extremum-principlespreserving schemes, as those developed in $30,41,12,27,19,28,35,31$, is to compute for each interior face $\sigma \in \mathcal{E}_{\text {int }}$, with $\mathcal{T}_{\sigma}=\{K, L\}$, two consistent linear flux approximations, $\tilde{F}_{K, \sigma}(u)$ and $\tilde{F}_{L, \sigma}(u)$, which depend on the unknown $u \in H_{\mathcal{T}}(\Omega)$, and to define the final flux $F_{K, \sigma}(u, u)$ as a convex combination (with weights $\mu_{K, \sigma}, \mu_{L, \sigma}$ that also depend on $u$ ) of these linear fluxes:

$$
\begin{gathered}
F_{K, \sigma}(u, u)=\mu_{K, \sigma}(u) \tilde{F}_{K, \sigma}(u)-\mu_{L, \sigma}(u) \tilde{F}_{L, \sigma}(u), \\
\text { with } \mu_{K, \sigma}(u) \geq 0, \mu_{L, \sigma}(u) \geq 0 \text { and } \mu_{K, \sigma}(u)+\mu_{L, \sigma}(u)=1,
\end{gathered}
$$


where the linear fluxes are defined by

$$
\tilde{F}_{K, \sigma}(u)=|\sigma| \sum_{\sigma^{\prime} \in \mathcal{S}_{K, \sigma}} \alpha_{K, \sigma \sigma^{\prime}}\left(I_{\sigma^{\prime}} u-u_{K}\right),
$$

where $\mathcal{S}_{K, \sigma}$ denotes the face stencil and $I_{\sigma^{\prime}} \in \mathcal{L}\left(H_{\mathcal{T}}(\Omega) ; \mathbb{P}^{0}(\sigma)\right)$ a trace reconstruction operator according to Definition 5. The stencil and the coefficients $\alpha_{K, \sigma \sigma^{\prime}}$ are determined by the conormal decomposition:

$$
\boldsymbol{\Lambda}_{K} \mathbf{n}_{K, \sigma}=\sum_{\sigma^{\prime} \in \mathcal{S}_{K, \sigma}} \alpha_{K, \sigma \sigma^{\prime}}\left(\mathbf{x}_{\sigma^{\prime}}-\mathbf{x}_{K}\right)
$$

This means that the conormal $\boldsymbol{\Lambda}_{K} \mathbf{n}_{K, \sigma}$ is decomposed into a basis $\left(\mathbf{x}_{\sigma^{\prime}}-\mathbf{x}_{K}\right)_{\left\{\sigma^{\prime} \in \mathcal{S}_{K, \sigma}\right\}}$ with coefficients $\left(\alpha_{K, \sigma \sigma^{\prime}}\right)_{\left\{\sigma^{\prime} \in \mathcal{S}_{K, \sigma}\right\}}$ which are computed to be non-negative, i.e. $\alpha_{K, \sigma \sigma^{\prime}} \geq 0$ if possible. By only including neighboring cells into the face stencils, the non-negativity cannot always be guaranteed. In [34, 36], we have thus introduced the idea of formulating the conormal decomposition as an optimization problem. The authors of [39] solve this issue by also including non-neighboring cells. For further details the reader is referred to the given references. By using this conormal decomposition, it can be shown that the linear sub-fluxes (74) are strongly consistent if the trace reconstruction operators $\left\{I_{\sigma^{\prime}}\right\}_{\sigma \in \mathcal{E}}$ are of second order accuracy 34].

For any $K \in \mathcal{T}, \sigma \in \mathcal{E}_{K} \cap \mathcal{E}_{\text {int }}$ and $L \in \mathcal{T}$ such that $\mathcal{T}_{\sigma}=\{K, L\}$, we thus get from (73) the numerical flux function $F_{K, \sigma}(\cdot, \cdot)$, defined for all $(u, v) \in\left[H_{\mathcal{T}}(\Omega)\right]^{2}$ by

$$
F_{K, \sigma}(u, v)=\mu_{K, \sigma}(u) \tilde{F}_{K, \sigma}(v)-\mu_{L, \sigma}(u) \tilde{F}_{L, \sigma}(v) .
$$

These flux functions are constructed to be conservative, such that equation (27) holds.

In the following, different choices for the weights $\mu_{K, \sigma}, \mu_{L, \sigma}$ are presented for the family of schemes (73). Moreover, a general trace reconstruction operator $I_{\sigma}$ (see Definition 5), which is needed for the derivation of the different nonlinear schemes, is introduced and given for each face $\sigma \in \mathcal{E}_{\text {int }}$ by

$$
I_{\sigma} u=\sum_{M \in \mathcal{I}_{\sigma}} \omega_{M, \sigma} u_{M}, \quad \sum_{M \in \mathcal{I}_{\sigma}} \omega_{M, \sigma}=1, \quad \omega_{M, \sigma} \geq 0,
$$

where the subset $\mathcal{I}_{\sigma} \subset \mathcal{T}$ stands for the interpolation index set (see [34] for further details).

\section{AvgMPFA scheme}

The most simple choice of coefficients is $\mu_{K, \sigma}=\mu_{L, \sigma}=0.5$ resulting in a linear finite volume scheme, which is in the following called AvgMPFA.

\section{NLTPFA scheme}

To derive a nonlinear two-point flux approximation (NLTPFA), the different terms are reordered such that the flux is written as

$$
F_{K, \sigma}(u)=t_{L, \sigma}(u) u_{L}-t_{K, \sigma}(u) u_{K}-\underbrace{\left(\mu_{L, \sigma}(u) \lambda_{L, \sigma}(u)-\mu_{K, \sigma}(u) \lambda_{K, \sigma}(u)\right)}_{\stackrel{\text { def }}{=} R_{K, \sigma}(u)},
$$

with the transmissibilities

$$
\begin{aligned}
& t_{K, \sigma}(u)=|\sigma|\left(\mu_{K, \sigma}(u) \sum_{\sigma^{\prime} \in \mathcal{S}_{K, \sigma}} \sum_{M \in\left\{\mathcal{I}_{\sigma^{\prime}} \backslash\{K\}\right\}} \alpha_{K, \sigma \sigma^{\prime}} \omega_{M, \sigma^{\prime}}+\mu_{L, \sigma}(u) \sum_{\sigma^{\prime} \in \mathcal{S}_{L, \sigma}} \sum_{M \in\left\{\mathcal{I}_{\sigma^{\prime}} \cap\{K\}\right\}} \alpha_{L, \sigma \sigma^{\prime}} \omega_{M, \sigma^{\prime}}\right) \\
& t_{L, \sigma}(u)=|\sigma|\left(\mu_{L, \sigma}(u) \sum_{\sigma^{\prime} \in \mathcal{S}_{L, \sigma}} \sum_{M \in\left\{\mathcal{I}_{\sigma^{\prime}} \backslash\{L\}\right\}} \alpha_{L, \sigma \sigma^{\prime}} \omega_{M, \sigma^{\prime}}+\mu_{K, \sigma}(u) \sum_{\sigma^{\prime} \in \mathcal{S}_{K, \sigma}} \sum_{M \in\left\{\mathcal{I}_{\sigma^{\prime}} \cap\{L\}\right\}} \alpha_{K, \sigma \sigma^{\prime}} \omega_{M, \sigma^{\prime}}\right)
\end{aligned}
$$


and

$$
\begin{aligned}
& \lambda_{K, \sigma}(v)=|\sigma| \sum_{\sigma^{\prime} \in \mathcal{S}_{K, \sigma}} \sum_{M \in\left\{\mathcal{I}_{\sigma^{\prime}} \backslash\{K, L\}\right\}} \alpha_{K, \sigma \sigma^{\prime}} \omega_{M, \sigma^{\prime}} v_{M}, \\
& \lambda_{L, \sigma}(v)=|\sigma| \sum_{\sigma^{\prime} \in \mathcal{S}_{L, \sigma}} \sum_{M \in\left\{\mathcal{I}_{\sigma^{\prime}} \backslash\{K, L\}\right\}} \alpha_{L, \sigma \sigma^{\prime}} \omega_{M, \sigma^{\prime}} v_{M} .
\end{aligned}
$$

The idea of the NLTPFA scheme is to choose the weights such that $R_{K, \sigma}(u)=0$. From a numerical point of view, it is sufficient that $\left|R_{K, \sigma}(u)\right| \leq \epsilon$. Under the assumption that $\lambda_{K, \sigma} \lambda_{L, \sigma} \geq 0$, this can be ensured, by taking

$$
\mu_{K, \sigma}(u)=\frac{\left|\lambda_{L, \sigma}(u)\right|+\epsilon}{\left|\lambda_{K, \sigma}(u)\right|+\left|\lambda_{L, \sigma}(u)\right|+2 \epsilon}, \quad \mu_{L, \sigma}(u)=\frac{\left|\lambda_{K, \sigma}(u)\right|+\epsilon}{\left|\lambda_{K, \sigma}(u)\right|+\left|\lambda_{L, \sigma}(u)\right|+2 \epsilon} .
$$

With this, the residual term is given as

$$
R_{K, \sigma}(u)=\epsilon \frac{\lambda_{L, \sigma}(u)-\lambda_{K, \sigma}(u)}{\left|\lambda_{K, \sigma}(u)\right|+\left|\lambda_{L, \sigma}(u)\right|+2 \epsilon},
$$

for which it holds that

$$
\left|R_{K, \sigma}(u)\right| \leq \epsilon .
$$

Instead of directly neglecting the term $R_{K, \sigma}(u)$, we will incorporate it partly into $t_{K, \sigma}, t_{L, \sigma}$ and then only neglect a smaller part $R_{K, \sigma}^{\epsilon}(u)$ for which it holds that $\left|R_{K, \sigma}^{\epsilon}(u)\right| \leq\left|R_{K, \sigma}(u)\right|$. Details can be found in 36. This results in the final fluxes

$$
F_{K, \sigma}(u, u)=\tilde{t}_{L, \sigma}(u) u_{L}-\tilde{t}_{K, \sigma}(u) u_{K},
$$

with the new transmissibilities $\tilde{t}_{K, \sigma}, \tilde{t}_{L, \sigma}$ which are greater or equal to the corresponding $t_{K, \sigma}, t_{L, \sigma}$ Therefore, the positivity of the new transmissibilities directly follow from the positivity of the old ones. Here, $\epsilon$ is chosen such that $0<\epsilon \leq h_{\mathcal{D}} \min _{\sigma \in \mathcal{E}}|\sigma|$.

\section{NLMPFA scheme}

A nonlinear multi-point flux approximation (NLMPFA) is derived by splitting the linear fluxes into a two-point part and a residual flux part as follows

$$
\begin{aligned}
\tilde{F}_{K, \sigma}(u) & =|\sigma| \beta_{\sigma}\left(u_{L}-u_{K}\right)+\tilde{F}_{K, \sigma}^{\mathrm{res}}(u), \\
\tilde{F}_{L, \sigma}(u) & =|\sigma| \beta_{\sigma}\left(u_{K}-u_{L}\right)+\tilde{F}_{L, \sigma}^{\mathrm{res}}(u),
\end{aligned}
$$

with $\beta_{\sigma}=\min \left(\alpha_{K, \sigma \sigma} \omega_{L, \sigma}, \alpha_{L, \sigma \sigma} \omega_{K, \sigma}\right)$ and $\tilde{F}_{K, \sigma}^{\text {res }}, \tilde{F}_{L, \sigma}^{\text {res }}$ defined by

$$
\begin{aligned}
\tilde{F}_{K, \sigma}^{\mathrm{res}}(v)= & |\sigma|\left(\alpha_{K, \sigma \sigma} \omega_{L, \sigma}-\beta_{\sigma}\right)\left(v_{L}-v_{K}\right)+|\sigma| \alpha_{K, \sigma \sigma} \sum_{M \in\left\{\mathcal{I}_{\sigma} \backslash\{L\}\right\}} \omega_{M, \sigma}\left(v_{M}-v_{K}\right) \\
& +\sum_{\sigma^{\prime} \in\left\{\mathcal{S}_{K, \sigma} \backslash\{\sigma\}\right\}}|\sigma| \alpha_{K, \sigma \sigma^{\prime}}\left(I_{\sigma^{\prime}} v-v_{K}\right), \\
\tilde{F}_{L, \sigma}^{\mathrm{res}}(v)= & |\sigma|\left(\alpha_{L, \sigma \sigma} \omega_{K, \sigma}-\beta_{\sigma}\right)\left(v_{K}-v_{L}\right)+|\sigma| \alpha_{L, \sigma \sigma} \sum_{M \in\left\{\mathcal{I}_{\sigma} \backslash\{K\}\right\}} \omega_{M, \sigma}\left(v_{M}-v_{L}\right) \\
& +\sum_{\sigma^{\prime} \in\left\{\mathcal{S}_{L, \sigma} \backslash\{\sigma\}\right\}}|\sigma| \alpha_{L, \sigma \sigma^{\prime}}\left(I_{\sigma^{\prime}} v-v_{L}\right) .
\end{aligned}
$$

Here, the weights are chosen as

$$
\begin{array}{ll}
\mu_{K, \sigma}(u)=\frac{\left|\tilde{F}_{L, \sigma}^{\mathrm{res}}\right|}{\left|\tilde{F}_{K, \sigma}^{\mathrm{res}}\right|+\left|\tilde{F}_{L, \sigma}^{\mathrm{res}}\right|}, \mu_{L, \sigma}(u)=\frac{\left|\tilde{F}_{K, \sigma}^{\mathrm{res}}\right|}{\left|\tilde{F}_{K, \sigma}^{\mathrm{res}}\right|+\left|\tilde{F}_{L, \sigma}^{\mathrm{res}}\right|}, & \text { if }\left|\tilde{F}_{K, \sigma}^{\mathrm{res}}(u)\right|+\left|\tilde{F}_{L, \sigma}^{\mathrm{res}}(u)\right|>0, \\
\mu_{K, \sigma}(u)=0.5, \mu_{L, \sigma}(u)=0.5, & \text { if }\left|\tilde{F}_{K, \sigma}^{\mathrm{res}}(u)\right|+\left|\tilde{F}_{L, \sigma}^{\mathrm{res}}(u)\right|=0,
\end{array}
$$


which results in

$$
\begin{aligned}
F_{K, \sigma} & =|\sigma| \beta_{\sigma}\left(u_{L}-u_{K}\right)+\mu_{K, \sigma}\left(1-\operatorname{sign}\left(\tilde{F}_{K, \sigma}^{\text {res }} \tilde{F}_{L, \sigma}^{\text {res }}\right)\right) \tilde{F}_{K, \sigma}^{\text {res }}, \\
F_{L, \sigma} & =|\sigma| \beta_{\sigma}\left(v_{K}-v_{L}\right)+\mu_{L, \sigma}\left(1-\operatorname{sign}\left(\tilde{F}_{K, \sigma}^{\text {res }} \tilde{F}_{L, \sigma}^{\text {res }}\right)\right) \tilde{F}_{L, \sigma}^{\text {res }} .
\end{aligned}
$$

As shown in 34, these fluxes are continuous because for $\left|\tilde{F}_{K, \sigma}^{\text {res }}(u)\right|+\left|\tilde{F}_{L, \sigma}^{\text {res }}(u)\right|=0$ they are independent of $\mu_{K, \sigma}, \mu_{L, \sigma}$. Furthermore, the constraint $\beta_{\sigma}>0$ is considered in the conormal decomposition, see 34 for more details.

\subsection{Properties}

In this section, some properties of fluxes belonging to the family 73 are summarized. First, the consistency and continuity of the fluxes is discussed, which allows the prove of (P2) and (P4)] In the second part, it is shown that the NLTPFA is positivity-preserving, whereas the NLMPFA prevents saturation under- and overshoots.

\subsubsection{Consistency and continuity}

The consistency property $(\overline{\mathrm{P}} 4)$ for the form $a$ follows from the strong consistency of the sub-fluxes $\tilde{F}_{K, \sigma}$, see Corollary 1 and 2 in 34 .

We now show that the continuity property (P2) holds for the fluxes 73$)$ and therefore also for the AvgMPFA, NLTPFA, and NLMPFA schemes.

Proposition 2 (Continuity) Let $\mathcal{D}$ be a space discretization matching Definition $2, \chi: \mathbb{R} \mapsto \mathbb{R} a$ bounded function, $\alpha \in H_{\Delta}(\Omega)$, and $a_{\mathcal{T}, \chi, \alpha}$ be the form defined by $(32)$ with the fluxes given by $(73)-(74)$. Then there exists a constant $C_{15}$ such that for all $(u, v, w) \in\left[H_{\mathcal{T}}(\Omega)\right]^{3}$

$$
\left|a_{\mathcal{T}, \chi, \alpha}(u, v, w)\right| \leq C_{15}\|v\|_{\mathcal{T}}\|w\|_{\mathcal{T}} .
$$

Proof Using the flux expression $(73)$, rearranging terms, and applying the Cauchy-Schwarz inequality yield

$$
\begin{aligned}
\left|a_{\mathcal{T}, \chi, \alpha}(u, v, w)\right| & =\left|\sum_{K \in \mathcal{T}} \sum_{\sigma \in \mathcal{E}_{K}} \chi\left(\alpha_{\sigma}\right) \tilde{F}_{K, \sigma}(v)\left(\Upsilon_{u, \sigma} w-w_{K}\right)\right| \\
& \leq\|w\|_{\mathcal{T}, \Upsilon_{\mu}}\|\chi\|_{\infty}\left(\sum_{K \in \mathcal{T}} \sum_{\sigma \in \mathcal{E}_{K}} \frac{d_{K, \sigma}}{|\sigma|}\left|\tilde{F}_{k, \sigma}\right|^{2}\right)^{\frac{1}{2}}
\end{aligned}
$$

with $\Upsilon_{\mu, \sigma}$ the trace reconstruction operator defined by 200 with $\theta_{K, \sigma}=\mu_{K, \sigma}(u)$. Inserting the subfluxes 74, using the fact that $\left|\alpha_{K, \sigma \sigma^{\prime}}\right| \leq \frac{C_{\alpha} \beta_{0}}{d_{K, \sigma^{\prime}}}$ (see equation (52) in 34]), the Cauchy-Schwarz inequality together with the fact that $\mathcal{S}_{K, \sigma} \subset \mathcal{E}_{K}$, and equation 116 to deduce

$$
\begin{aligned}
& \left(\sum_{K \in \mathcal{T}} \sum_{\sigma \in \mathcal{E}_{K}} \frac{d_{K, \sigma}}{|\sigma|}\left(|\sigma| \sum_{\sigma^{\prime} \in \mathcal{S}_{K, \sigma}}\left|\alpha_{K, \sigma \sigma^{\prime}}\right|\left|I_{\sigma^{\prime}} v-v_{K}\right|\right)^{2}\right)^{\frac{1}{2}} \\
& \leq C_{\alpha} \beta_{0}\left(\sum_{K \in \mathcal{T}} \sum_{\sigma \in \mathcal{E}_{K}} d_{K, \sigma}|\sigma|\left\{\left(\sum_{\sigma^{\prime} \in \mathcal{E}_{K}} \frac{1}{d_{K, \sigma^{\prime}}\left|\sigma^{\prime}\right|}\right)\left(\sum_{\sigma^{\prime} \in \mathcal{E}_{K}} \frac{\left|\sigma^{\prime}\right|}{d_{K, \sigma^{\prime}}}\left|I_{\sigma^{\prime}} v-v_{K}\right|^{2}\right)\right\}\right)^{\frac{1}{2}} \\
& \leq \sqrt{\frac{d \zeta_{1}}{\zeta_{2} \zeta_{3}}} C_{\alpha} \beta_{0}\|v\|_{\mathcal{T}, I} .
\end{aligned}
$$


Gathering the previous results give:

$$
\left|a_{\mathcal{T}, \chi, \alpha}(u, v, w)\right| \leq\|\chi\|_{\infty} \sqrt{\frac{d \zeta_{1}}{\zeta_{2} \zeta_{3}}} C_{\alpha} \beta_{0}\|v\|_{\mathcal{T}, I}\|w\|_{\mathcal{T}, \Upsilon_{\mu}}
$$

Using the equivalence of the discrete $H^{1}$-norms (see Lemma 1 in [34]), the previous inequality implies 88 .

\subsubsection{Saturation bounds}

In this section, it is shown that, in contrast to general linear schemes, the NLTPFA scheme prevents saturation undershoots, whereas the NLMPFA scheme prevents both under- and overshoots.

Proposition 3 () Assume that Hypotheses 1 hold. Let $\mathscr{D}$ be a space-time discretization matching Definition 3 and $u \in H_{\mathscr{T}}(\Omega \times(0, T))$ a solution to (28) with the fluxes given by (73)-(74). Let us assume that all coefficients $\alpha_{K, \sigma \sigma^{\prime}}, \omega_{M, \sigma}$ of the conormal decomposition $(75)$ and of the reconstruction operator (77) are non-negative. Then, for all $n \in \llbracket 0, N \rrbracket$ and for all $K \in \mathcal{T}$, we have, in the case of the NLMPFA fluxes (87),

$$
0 \leq u_{K}^{(n)} \leq 1
$$

and in the case of the NLTPFA fluxes 83,

$$
u_{K}^{(n)} \geq 0 .
$$

Proof For all $K \in \mathcal{T}$, we rewrite equation (28a) as

$$
u_{K}^{(n)}=u_{K}^{(n+1)}+\frac{\delta t^{\left(n+\frac{1}{2}\right)}}{|K|}\left(\begin{array}{l}
f_{1}\left(u_{K}^{(n+1)}\right) \sum_{\sigma \in \mathcal{E}_{K}}\left(\mathbf{v}_{K, \sigma}^{(n+1)}\right)^{+}+\sum_{\sigma \in \mathcal{E}_{K}} f_{1}\left(u_{L}^{(n+1)}\right)\left(\mathbf{v}_{K, \sigma}^{(n+1)}\right)^{-} \\
+f_{1}\left(u_{K}^{(n+1)}\right) \sum_{\sigma \in \mathcal{E}_{K}} \lambda_{2}\left(u_{L}^{(n+1)}\right)\left(\left(\varrho_{1}-\varrho_{2}\right) G_{K, \sigma}\right)^{+} \\
+\lambda_{2}\left(u_{K}^{(n+1)}\right) \sum_{\sigma \in \mathcal{E}_{K}}^{\sigma(n+1)} f_{1}\left(u_{L}^{(n+1)}\right)\left(\left(\varrho_{1}-\varrho_{2}\right) G_{K, \sigma}\right)^{-} \\
-\sum_{\sigma \in \mathcal{E}_{K}} F_{K, \sigma}\left(\psi\left(u^{(n+1)}\right), \psi\left(u^{(n+1)}\right)\right) \\
-|K|\left(f_{1}\left(c_{K}\right) s_{K}^{+}-f_{1}\left(u_{K}^{(n+1)}\right) s_{K}^{-}\right)
\end{array}\right)
$$

where $a^{+}=\max (a, 0)$ and $a^{-}=\min (a, 0)$.

Let us first prove that the saturations are positive at all time steps. By assumption, this is the case for the initial ones. Let us assume this property is valid up to some time step $n$ and let us prove that it then also holds for the time step $n+1$. Let us assume that this is not the case, such that there exists at least one cell $K$ for which $u_{K}^{(n+1)}<0$ and such that $u_{K}^{(n+1)}=\min _{M \in \mathcal{T}}\left(u_{M}^{(n+1)}\right)$. Using the extension of the function $\lambda_{1}$ to 0 on $(-\infty, 0]$ for which we infer $f_{1}\left(u_{K}^{(n+1)}\right)=0$, and the fact that $s^{+} \geq 0$, we deduce that

$$
u_{K}^{(n)}<-\frac{\delta t^{\left(n+\frac{1}{2}\right)}}{|K|} \sum_{\sigma \in \mathcal{E}_{K}} F_{K, \sigma}\left(\psi\left(u^{(n+1)}\right), \psi\left(u^{(n+1)}\right)\right) .
$$

Let us now study the sign of the right hand side in the last inequality. We first consider the case of the NLMPFA fluxes (87) which can be rewritten as

$$
F_{K, \sigma}(u, u)=\sum_{M} \omega_{K, \sigma, M}(u)\left(u_{M}-u_{K}\right), \quad \omega_{K, \sigma, M}(u) \geq 0 .
$$

Since $u_{K}^{(n+1)}=\min _{M \in \mathcal{T}}\left(u_{M}^{(n+1)}\right)$ and $\psi$ is an increasing function, it follows that

$$
-\frac{\delta t^{\left(n+\frac{1}{2}\right)}}{|K|} \sum_{\sigma \in \mathcal{E}_{K}} F_{K, \sigma}\left(\psi\left(u^{(n+1)}\right), \psi\left(u^{(n+1)}\right)\right) \leq 0,
$$


leading to $u_{K}^{(n)}<0$ which is in contradiction with our assumption. Let us now prove the positivity of the saturations obtained with the NLTPFA fluxes 837 . For this, we use the fact that inequality 92 holds for each cell $M \in \mathcal{T}$ for which $u_{M}^{(n+1)}<0$. Thus, summing up over the set $\mathcal{T}^{-} \stackrel{\text { def }}{=}\{M \in$ $\left.\mathcal{T} \mid u_{M}^{(n+1)}<0\right\}$ yields

$$
\begin{aligned}
\sum_{K \in \mathcal{T}^{-}} \frac{|K|}{\delta t^{\left(n+\frac{1}{2}\right)}} u_{K}^{(n)}< & -\sum_{K \in \mathcal{T}^{-}} \sum_{\sigma \in \mathcal{E}_{K}} F_{K, \sigma}\left(\psi\left(u^{(n+1)}\right), \psi\left(u^{(n+1)}\right)\right) \\
= & -\sum_{\sigma \in \mathcal{E}_{\text {int }}, \mathcal{T}_{\sigma}=\{K, L\}, K \in \mathcal{T}^{-}, L \notin \mathcal{T}^{-}} F_{K, \sigma}\left(\psi\left(u^{(n+1)}\right), \psi\left(u^{(n+1)}\right)\right) \\
& -\sum_{\sigma \in \mathcal{E}_{\text {ext }}, \mathcal{T}_{\sigma}=\{K\}, K \in \mathcal{T}^{-}} F_{K, \sigma}\left(\psi\left(u^{(n+1)}\right), \psi\left(u^{(n+1)}\right)\right),
\end{aligned}
$$

where we have used the local flux conservation 27). Using the fact that $t_{K, \sigma}, t_{L, \sigma} \geq 0$ and that $\psi(u)<0$ for all $u<0$ it follows that

$$
F_{K, \sigma}\left(\psi\left(u^{(n+1)}\right), \psi\left(u^{(n+1)}\right)\right)=t_{L, \sigma}\left(\psi\left(u^{(n+1)}\right)\right) \psi\left(u_{L}^{(n+1)}\right)-t_{K, \sigma}\left(\psi\left(u^{(n+1)}\right)\right) \psi\left(u_{K}^{(n+1)}\right) \geq 0,
$$

for all $\sigma \in \mathcal{E}_{\text {int }}, \mathcal{T}_{\sigma}=\{K, L\}$ such that $K \in \mathcal{T}^{-}$and $L \notin \mathcal{T}^{-}$. For all $\sigma \in \mathcal{E}_{\text {ext }}, \mathcal{T}_{\sigma}=\{K\}$ such that $K \in \mathcal{T}^{-}$it also follows that $F_{K, \sigma} \geq 0$ because of homogeneous zero Dirichlet conditions for which $\psi(0)=0$. This leads to a contradiction, which therefore shows the positivity of the NLTPFA solution. Now let us prove that the saturations obtained with the NLMPFA fluxes are smaller than one at all time steps. As before, let us assume this property is valid up to some time step $n$ and let us prove that it then also holds for the time step $n+1$. We write equation (28b) as

$$
\frac{\delta t^{\left(n+\frac{1}{2}\right)}}{|K|}\left(\sum_{\sigma \in \mathcal{E}_{K}} \mathbf{v}_{K, \sigma}^{(n+1)}-|K|\left(s_{K}^{+}-s_{K}^{-}\right)\right)=0 .
$$

We substract equation (91) from 977) and using the fact that $f_{1}+f_{2}=1$ to obtain for all $K \in \mathcal{T}$

$$
1-u_{K}^{(n)}=1-u_{K}^{(n+1)}+\frac{\delta t^{\left(n+\frac{1}{2}\right)}}{|K|}\left(\begin{array}{l}
f_{2}\left(u_{K}^{(n+1)}\right) \sum_{\sigma \in \mathcal{E}_{K}}\left(\mathbf{v}_{K, \sigma}^{(n+1)}\right)^{+}+\sum_{\sigma \in \mathcal{E}_{K}} f_{2}\left(u_{L}^{(n+1)}\right)\left(\mathbf{v}_{K, \sigma}^{(n+1)}\right)^{-} \\
-f_{1}\left(u_{K}^{(n+1)}\right) \sum_{\sigma \in \mathcal{E}_{K}} \lambda_{2}\left(u_{L}^{(n+1)}\right)\left(\left(\varrho_{1}-\varrho_{2}\right) G_{K, \sigma}\right)^{+} \\
-\lambda_{2}\left(u_{K}^{(n+1)}\right) \sum_{\sigma \in \mathcal{E}_{K}}^{\sigma(n+1)} f_{1}\left(u_{L}^{(n+1)}\right)\left(\left(\varrho_{1}-\varrho_{2}\right) G_{K, \sigma}\right)^{-} \\
+\sum_{\sigma \in \mathcal{E}_{K}} F_{K, \sigma}\left(\psi\left(u^{(n+1)}\right),\left(u^{(n+1)}\right)\right) \\
-|K|\left(f_{2}\left(c_{K}\right) s_{K}^{+}-f_{2}\left(u_{K}^{(n+1)}\right) s_{K}^{-}\right)
\end{array}\right)
$$

which implies

$$
1-u_{K}^{(n)} \leq 1-u_{K}^{(n+1)}+\frac{\delta t^{\left(n+\frac{1}{2}\right)}}{|K|}\left(\begin{array}{l}
f_{2}\left(u_{K}^{(n+1)}\right) \sum_{\sigma \in \mathcal{E}_{K}}\left(\mathbf{v}_{K, \sigma}^{(n+1)}\right)^{+} \\
-\lambda_{2}\left(u_{K}^{(n+1)}\right) \sum_{\sigma \in \mathcal{E}_{K}} f_{1}\left(u_{L}^{(n+1)}\right)\left(\left(\varrho_{1}-\varrho_{2}\right) G_{K, \sigma}\right)^{-} \\
+\sum_{\sigma \in \mathcal{E}_{K}} F_{K, \sigma}\left(\psi\left(u^{(n+1)}\right), \psi\left(u^{(n+1)}\right)\right) \\
+|K| f_{2}\left(u_{K}^{(n+1)}\right) s_{K}^{-}
\end{array}\right)
$$

Let us assume that there exists at least one cell $K$ for which $u_{K}^{(n+1)}>1$ and such that $u_{K}^{(n+1)}=$ $\max _{M \in \mathcal{T}}\left(u_{M}^{(n+1)}\right)$. Using the extension of the function $\lambda_{2}$ to 0 on $[1,+\infty)$ for which we infer $f_{2}\left(u_{K}^{(n+1)}\right)=$ $\lambda_{2}\left(u_{K}^{(n+1)}\right)=0$, we deduce from 99 that

$$
1-u_{K}^{(n)} \leq 1-u_{K}^{(n+1)}+\frac{\delta t^{\left(n+\frac{1}{2}\right)}}{|K|} \sum_{\sigma \in \mathcal{E}_{K}} F_{K, \sigma}\left(\psi\left(u^{(n+1)}\right), \psi\left(u^{(n+1)}\right)\right) .
$$


By using the expression of the NLMPFA fluxes $(93)$, since $u_{K}^{(n+1)}=\max _{M \in \mathcal{T}}\left(u_{M}^{(n+1)}\right)$ and $\psi$ is an increasing function, we then have

$$
1-u_{K}^{(n+1)}+\frac{\delta t^{\left(n+\frac{1}{2}\right)}}{|K|} \sum_{\sigma \in \mathcal{E}_{K}} F_{K, \sigma}\left(\psi\left(u^{(n+1)}\right), \psi\left(u^{(n+1)}\right)\right)<0,
$$

leading to $1-u_{K}^{(n)}<0$ which is in contradiction with our assumption.

\section{Numerical results}

In this section, the behavior of the above mentioned nonlinear finite volume schemes is investigated for different two-phase flow scenarios. NLTPFA denotes the scheme which is given by equation (83) and the weights (81), NLMPFA the scheme defined by equation (87) and the weights (86) with the additional constraints that $\alpha_{K, \sigma \sigma}, \alpha_{L, \sigma \sigma}>0$ and AvgMPFA the scheme with fluxes (76) and constant weights $\mu_{K, \sigma}=\mu_{L, \sigma}=0.5$. Further details can be found in 34, 36].

All simulations are performed using the open-source simulator $\mathrm{DuMu}^{\mathrm{x}}[25$, which comes in the form of an additional DUNE module 6]. Furthermore, for all grids, the dune-alugrid module [3] is used. A monolithic approach is applied, where Newton's method is used for solving the occurring nonlinear systems of equations. We use a relative stopping criterion for Newton's method, where the iteration loop is stopped if relative changes of the primary variables are below $10^{-8}$, see the $\mathrm{DuMu}^{\mathrm{x}}$ documentation for more information. Such a relative criterion does not require the recalculation of the residual, which is therefore more efficient.

In general, the exact solution is unknown. Therefore, we have to interpolate numerical solutions on fine grid levels to coarser ones to calculate convergence rates. There are several possibilities to do this. Here we present the one for which we have observed the least fluctuating rates. We are interested in the spatial and temporal convergence rates for the discrete space-time $L^{2}$-norm $\left(\|\cdot\|_{L^{2}(\Omega \times(0, T))}\right)$ and $L^{2}\left(0, T ; H^{1}(\Omega)\right)$-norm $\left(\|\cdot\|_{\mathscr{T}}\right)$. To calculate the convergence rates for increasing spatial refinement, a small time step size is fixed and the numerical solution on refinement level $m$ is interpolated on level $m-1,1 \leq m \leq m_{\max }$. This interpolation is done by calculating coefficients such that for each cell $K_{m-1} \in \mathcal{T}_{m-1}$ it holds that

$$
\mathbf{x}_{K_{m-1}}=\sum_{L_{m} \in \mathcal{T}_{m}, L_{m} \subset \bar{K}_{m-1}} \omega_{L_{m}} \mathbf{x}_{L_{m}} .
$$

With these coefficients, the solution $\left(u_{m}, p_{m}\right)$ on grid level $m$ is interpolated at the cell centers $\mathbf{x}_{K_{m-1}}$ of grid level $m-1$ as

$$
\left(u_{m}, p_{m}\right)\left(\mathbf{x}_{K_{m-1}}\right)=\sum_{L_{m} \in \mathcal{T}_{m}, L_{m} \subset \bar{K}_{m-1}} \omega_{L_{m}}\left(u_{L_{m}}, p_{L_{m}}\right),
$$

where $\left(u_{L_{m}}, p_{L_{m}}\right)$ are the given solution values on grid level $m$. Please note that the grid refinement is done such that $\mathbf{x}_{K_{m-1}} \in \operatorname{Conv}\left(\left\{\mathbf{x}_{L_{m}}\right\}_{L_{m} \in \mathcal{T}_{m}, L_{m} \subset \bar{K}_{m-1}}\right)$ for all $1 \leq m \leq m_{\max }$, which means that each center of a coarse cell is in the convex hull constructed by the centers of its child elements.

For calculating the temporal convergence rates, a reference solution $\left(u_{\text {ref }}, p_{\text {ref }}\right)$ is calculated using a small discretization length $h_{\mathcal{D} \text {,ref }}$ and a small time step size $\delta t_{\text {ref }}$. This reference solution is then mapped to the discrete time levels calculated for bigger time step sizes $\delta t_{i}$. These time step sizes are

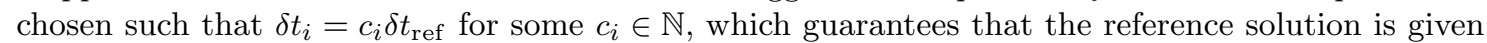
for the discrete times calculated for coarser time discretizations.

To guarantee that the assumptions (A6) (A7) are fulfilled, the mobilities are evaluated as

$$
\lambda_{i}(u)=\lambda_{i}(\max \{0, \min \{u, 1\}\}), \quad \forall u \in \mathbb{R}, i=1,2 .
$$

The linearization $(9)$ is also applied on $\psi$. Please note that this is a continuous extension of $\psi$ because $\psi(0)=0$. Furthermore, with this linearization, $\psi$ is strictly increasing for all $u \in \mathbb{R}$. If not explicitly 
mentioned, the scaling factor is chosen as $\Xi=1$. The influence of $\Xi$ on saturation overshoots is investigated in Section 6.1 .3

Furthermore, the coercivity property (34) is numerically investigated by using the following quantity

$$
e_{\mathcal{T}, m}\left(v_{m}\right) \stackrel{\text { def }}{=} \inf _{t \in(0, T)} \frac{a_{\mathcal{T}_{m}, \chi, \alpha}\left(v_{m}(t), v_{m}(t), v_{m}(t)\right)}{\left\|v_{m}(t)\right\|_{\mathcal{T}_{m}}^{2}}
$$

where $v_{m} \in\left\{\psi_{m}, p_{m}\right\}$ and $\chi=1$ due to the fact that the mobility functions are chosen such that $\lambda_{\mathrm{T}}=$ const for all considered test cases, which also means that $a_{\mathcal{T}_{m}, \chi, \alpha}$ is independent of $\alpha$. This is obviously not sufficient to show the coercivity of the considered schemes but it serves here as an indicator.

For simplicity, Neumann boundary conditions are defined for the phase velocities $\mathbf{v}_{1}, \mathbf{v}_{2}$. However, this can be equivalently written as

$$
\begin{aligned}
& \mathbf{v}_{1} \cdot \mathbf{n}=\nu_{1}, \\
& \mathbf{v}_{2} \cdot \mathbf{n}=\nu_{2}
\end{aligned} \quad \Longleftrightarrow \quad \begin{aligned}
& \left(f_{1} \mathbf{v}_{\mathrm{T}}-\boldsymbol{\Lambda} \nabla \psi+\left(\varrho_{1}-\varrho_{2}\right) f_{1} \lambda_{2} \boldsymbol{\Lambda} \mathbf{g}\right) \cdot \mathbf{n}=\nu_{1}, \\
& \mathbf{v}_{\mathrm{T}} \cdot \mathbf{n}=\nu_{1}+\nu_{2} .
\end{aligned}
$$

The first test case investigates the convergence behavior for a quasi one-dimensional setup without gravity effects, with a constant total velocity, and with homogeneous porosity and permeability. The second test case includes gravity effects and heterogeneities.

\subsection{Quasi one-dimensional test case}

In the first test case, we investigate a two-dimensional setup, with $\Omega=[0,1] \times[0,0.2]$, for which the solution is expected to be constant with respect to the $y$-coordinate, meaning that the solution profile is quasi one-dimensional. Gravity is neglected and there are no source or sink terms (i.e. $\left.s^{+}=s^{-}=0\right)$. The simulation time is $T=300 \mathrm{~s}$, the porosity is set to $\phi=0.3$, and the permeability is assumed to be homogeneous and isotropic $\Lambda=10^{-10} \mathbf{I}$. The setting and the grid that are used for this test case are shown in Figure 2 No-flow boundary conditions are set at the top and bottom boundaries, whereas Dirichlet conditions are set on the right boundary. For analyzing the convergence rates Neumann boundary conditions are set on the left boundary (situation (a)), this guarantees that, independent of the grid, the same amount of mass enters the domain on the left. For analyzing the saturation over- and undershoots, Dirichlet conditions are set on the left boundary (situation (b)). The grid shown in Figure 2 corresponds to a grid refinement level of zero (16x16 cells). The direct solver UMFPack 13] is used to solve the occurring linear systems of equations.

The densities of the fluids are set to $\varrho_{1}=1000.0 \mathrm{~kg} / \mathrm{m}^{3}, \varrho_{2}=1500.0 \mathrm{~kg} / \mathrm{m}^{3}$. The mobility functions are given by

$$
\lambda_{1}(u)=\frac{u^{2}}{\mu_{1}}, \quad \lambda_{2}(u)=\frac{1-u^{2}}{\mu_{2}}, \quad \forall u \in[0,1]
$$

where the dynamic fluid viscosities are set to $\mu_{1}=\mu_{2}=0.003 \mathrm{Pas}$. If $u \notin[0,1]$ formula (104) is used. A linear function is used for the capillary pressure, which is given as

$$
p_{c}(u)=10^{4}(1-u)
$$

For these functions, an analytical expression for $\psi$ can be derived:

$$
\psi(u)=\frac{10^{4}}{\mu_{1}}\left(\frac{1}{3} u^{3}-\frac{1}{5} u^{5}\right)
$$

which is used for all $u \in[0,1]$ and the linearization $(9)$ is utilized for all other values of $u$. 


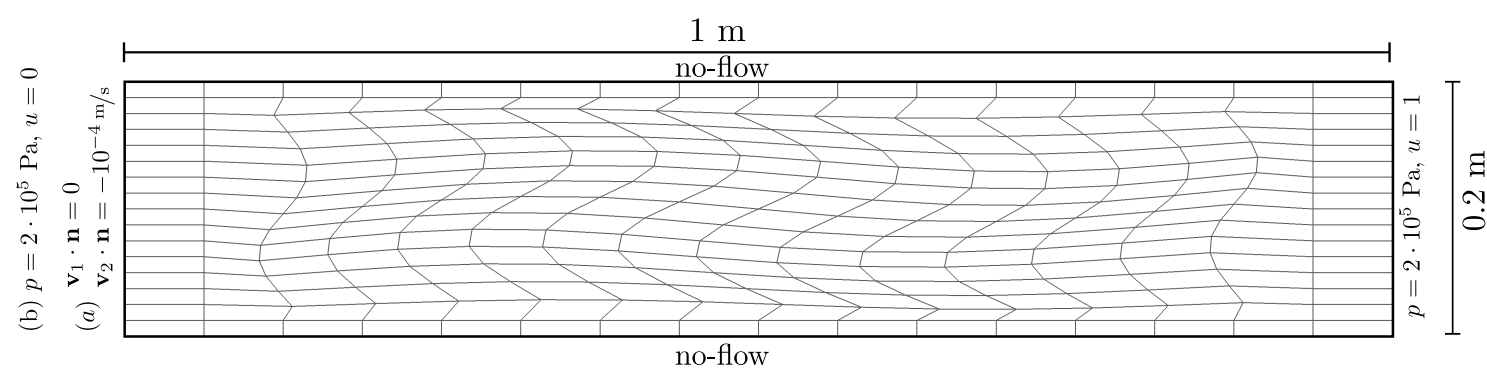

Fig. 2 Grid and setting that are used for the quasi one-dimensional test case. No-flow boundary conditions are set at the top and bottom boundaries, whereas Dirichlet conditions are set on the right boundary. Two different situations are considered on the left boundary: (a) Neumann conditions and (b) Dirichlet conditions.

\subsubsection{Spatial convergence}

In the following, the convergence rates are analyzed with increasing mesh refinement, where the maximum refinement level is $m_{\max }=4$ and a small time step size of $\delta t=0.05 \mathrm{~s}$ is used for all simulation runs. It is assumed that the domain is initially fully-saturated with the wetting fluid phase, i.e. $u_{\text {init }}(\mathbf{x})=1$, and the second fluid phase (non-wetting phase) enters the domain on the left (situation (a) in Figure 2). By neglecting gravity and assuming homogeneous data, the total velocity is constant for this quasi one-dimensional setup and therefore corresponds to a linear pressure profile. All schemes are able to exactly reproduce this linear profile. Therefore, only the convergence behavior with respect to $u$ and $\psi(u)$ is explored for this test case.

Figure 3 shows the saturation solution of the NLMPFA scheme for the grid with refinement level $m=3$ and the solution differences between two consecutive grid refinement levels. Table 1 and 2 list the discrete $L^{2}(\Omega \times(0, T))$-errors and $L^{2}\left(0, T ; H^{1}(\Omega)\right)$-errors together with the related convergence rates for $u$ and $\psi(u)$, respectively. For $u$, also the overshoots are shown. Additionally, Table 2 lists the total number of Newton iterations that are needed for the whole simulation run and the coercivity estimates calculated with formula (105).
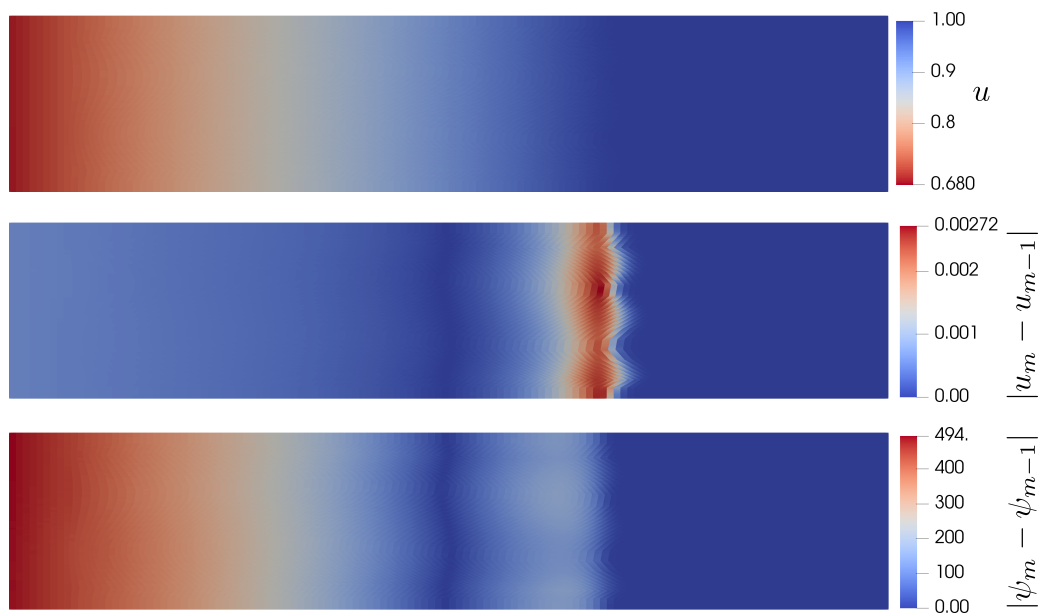

Fig. 3 Saturation solution of NLMPFA scheme and difference between fine solution $\left(u_{m}, \psi_{m}\right)$ and coarser solution $\left(u_{m-1}, \psi_{m-1}\right)$ for $m=4$ (mapping is done using formula 103).

In Table 1 it can be seen that all schemes converge with approximately first order with respect to the discrete $L^{2}(\Omega \times(0, T))$-norm and with a rate of approximately 0.3 with respect to the discrete $L^{2}\left(0, T ; H^{1}(\Omega)\right)$-norm for the variable $u$. In general, we cannot expect a higher rate since upwinding is used for the term $f_{1} \mathbf{v}_{\mathrm{T}}$. As expected, no saturation overshoots can be observed for the NLMPFA 
Table 1 Discrete space-time error norms and convergence rates $(c r)$ with respect to spatial discretization for quasi one-dimensional test case (situation (a) in Figure 2) for the variable $u$.

\begin{tabular}{c|c|cccccc} 
scheme & $m$ & $\left\|u_{m}-u_{m-1}\right\|_{L^{2}}$ & $c r$ & $\left\|u_{m}-u_{m-1}\right\| \mathscr{T}$ & $c r$ & $u_{\max }-1$ & $h_{\mathcal{D}}$ \\
\hline \multirow{4}{*}{ NLTPFA } & 1 & $1.55 \mathrm{e}-2$ & - & $6.57 \mathrm{e}-1$ & - & $8.17 \mathrm{e}-4$ & $9.25 \mathrm{e}-2$ \\
& 2 & $8.06 \mathrm{e}-3$ & 0.95 & $5.31 \mathrm{e}-1$ & 0.31 & $4.40 \mathrm{e}-4$ & $4.64 \mathrm{e}-2$ \\
& 3 & $4.06 \mathrm{e}-3$ & 0.99 & $4.13 \mathrm{e}-1$ & 0.36 & $1.59 \mathrm{e}-4$ & $2.32 \mathrm{e}-2$ \\
& 4 & $2.05 \mathrm{e}-3$ & 0.99 & $3.20 \mathrm{e}-1$ & 0.37 & $5.48 \mathrm{e}-5$ & $1.16 \mathrm{e}-2$ \\
\hline \multirow{5}{*}{ NLMPFA } & 1 & $2.37 \mathrm{e}-2$ & - & $4.10 \mathrm{e}-1$ & - & $-1.40 \mathrm{e}-5$ & $9.25 \mathrm{e}-2$ \\
& 2 & $1.49 \mathrm{e}-2$ & 0.67 & $3.95 \mathrm{e}-1$ & 0.06 & $-2.51 \mathrm{e}-9$ & $4.64 \mathrm{e}-2$ \\
& 3 & $7.77 \mathrm{e}-3$ & 0.94 & $3.37 \mathrm{e}-1$ & 0.23 & 0.00 & $2.32 \mathrm{e}-2$ \\
& 4 & $3.71 \mathrm{e}-3$ & 1.07 & $2.73 \mathrm{e}-1$ & 0.31 & 0.00 & $1.16 \mathrm{e}-2$ \\
\hline \multirow{5}{*}{ AvgMPFA } & 1 & $1.59 \mathrm{e}-2$ & - & $6.65 \mathrm{e}-1$ & - & $7.48 \mathrm{e}-4$ & $9.25 \mathrm{e}-2$ \\
& 2 & $8.21 \mathrm{e}-3$ & 0.96 & $5.34 \mathrm{e}-1$ & 0.32 & $4.18 \mathrm{e}-4$ & $4.64 \mathrm{e}-2$ \\
& 3 & $4.11 \mathrm{e}-3$ & 1.00 & $4.15 \mathrm{e}-1$ & 0.36 & $1.54 \mathrm{e}-4$ & $2.32 \mathrm{e}-2$ \\
& 4 & $2.06 \mathrm{e}-3$ & 1.00 & $3.21 \mathrm{e}-1$ & 0.37 & $5.41 \mathrm{e}-5$ & $1.16 \mathrm{e}-2$
\end{tabular}

Table 2 Discrete space-time error norms (scaled by the factor $\frac{1}{\psi(1)}$ ) and convergence rates $(c r)$ with respect to spatial discretization for quasi one-dimensional test case (situation (a) in Figure 2 for the variable $\psi(u)$.

\begin{tabular}{c|c|cccccc} 
scheme & $m$ & $\frac{1}{\psi(1)}\left\|\psi_{m}-\psi_{m-1}\right\|_{L^{2}}$ & $c r$ & $\frac{1}{\psi(1)}\left\|\psi_{m}-\psi_{m-1}\right\|_{\mathscr{T}}$ & $c r$ & $\frac{1}{\alpha_{0}} e_{\mathcal{T}, m}$ & $\mathrm{nIt}$ \\
\hline \multirow{5}{*}{ NLTPFA } & 1 & $1.64 \mathrm{e}-2$ & - & $2.23 \mathrm{e}-1$ & - & $3.72 \mathrm{e}-1$ & 12152 \\
& 2 & $8.20 \mathrm{e}-3$ & 1.00 & $1.26 \mathrm{e}-1$ & 0.83 & $3.66 \mathrm{e}-1$ & 17245 \\
& 3 & $4.01 \mathrm{e}-3$ & 1.03 & $6.36 \mathrm{e}-2$ & 0.99 & $3.62 \mathrm{e}-1$ & 18009 \\
& 4 & $1.97 \mathrm{e}-3$ & 1.03 & $3.09 \mathrm{e}-2$ & 1.04 & $3.61 \mathrm{e}-1$ & 18433 \\
\hline \multirow{3}{*}{ NLMPFA } & 1 & $2.82 \mathrm{e}-2$ & - & $3.23 \mathrm{e}-1$ & - & $4.07 \mathrm{e}-1$ & 12029 \\
& 2 & $1.49 \mathrm{e}-2$ & 0.92 & $1.94 \mathrm{e}-1$ & 0.74 & $3.77 \mathrm{e}-1$ & 12231 \\
& 3 & $6.60 \mathrm{e}-3$ & 1.18 & $9.32 \mathrm{e}-2$ & 1.06 & $3.66 \mathrm{e}-1$ & 12894 \\
& 4 & $2.81 \mathrm{e}-3$ & 1.23 & $4.11 \mathrm{e}-2$ & 1.18 & $3.62 \mathrm{e}-1$ & 13677 \\
\hline \multirow{3}{*}{ AvgMPFA } & 1 & $1.69 \mathrm{e}-2$ & - & $2.30 \mathrm{e}-1$ & - & $3.75 \mathrm{e}-1$ & 12157 \\
& 2 & $8.39 \mathrm{e}-3$ & 1.02 & $1.29 \mathrm{e}-1$ & 0.84 & $3.67 \mathrm{e}-1$ & 17234 \\
& 3 & $4.07 \mathrm{e}-3$ & 1.04 & $6.45 \mathrm{e}-2$ & 1.00 & $3.63 \mathrm{e}-1$ & 18017 \\
& 4 & $1.99 \mathrm{e}-3$ & 1.04 & $3.11 \mathrm{e}-2$ & 1.05 & $3.61 \mathrm{e}-1$ & 18472
\end{tabular}

scheme, whereas the NLTPFA and AvgMPFA schemes produce small overshoots (for further discussion of over- and undershoots see Section 6.1.3. For $\psi$ first order convergence is observed with respect to both discrete norms. A possible reason for the better convergence rate with respect to the discrete $L^{2}\left(0, T ; H^{1}(\Omega)\right)$-norm is the fact that $\psi^{\prime}(1)=0$ such that the regions with high saturation errors do not necessarily result in regions with high $\psi$-errors, see Figure 3 . In terms of Newton convergence, the NLMPFA scheme shows a better behavior compared to the other schemes. This is probably because the NLMPFA scheme does not produce any over- and undershoots and thus the regularization of $\psi(9)$ has no influence on the Newton convergence, whereas it may influence the Newton behavior of the other schemes. The coercivity estimates shown in Table 2 indicate that all schemes are coercive for this test case.

\subsubsection{Temporal convergence}

In this section, the convergence rates with respect to time discretization is investigated. Here, a fine grid resolution $\left(128 \times 128\right.$ cells with $\left.h_{\mathcal{D}}=2.32 \mathrm{e}-2\right)$ is chosen and a reference solution is calculated on this grid with a small time step size of $\delta t_{\mathrm{ref}}=0.05 \mathrm{~s}$. The results are listed in Table 3 and 4 . Again, the results of $p$ are not shown because the linear solution profile is exactly reproduced. Here, a first order convergence rate for the discrete $L^{2}(\Omega \times(0, T))$-norm is observed for all schemes, which is as expected for the implicit Euler scheme. Furthermore, in Table 3 it can be seen that the saturation overshoots of the NLTPFA and AvgMPFA become larger for smaller time step sizes. This could be one reason why the convergence rates with respect to the discrete $L^{2}\left(0, T ; H^{1}(\Omega)\right)$-norm are lower for the NLTPFA and AvgMPFA schemes compared to the NLMPFA scheme. 
Table 3 Discrete space-time error norms and convergence rates $(c r)$ with respect to temporal discretization for quasi one-dimensional test case (situation (a) in Figure 2 for the variable $u$.

\begin{tabular}{c|c|cccccc} 
scheme & $m$ & $\left\|u_{m}-u_{\text {ref }}\right\|_{L^{2}}$ & $c r$ & $\left\|u_{m}-u_{\text {ref }}\right\| \mathscr{T}$ & $c r$ & $u_{\text {max }}-1$ & $\delta t$ \\
\hline \multirow{4}{*}{ NLTPFA } & 0 & $9.10 \mathrm{e}-03$ & - & $7.81 \mathrm{e}-01$ & - & $1.09 \mathrm{e}-05$ & 4.00 \\
& 1 & $7.01 \mathrm{e}-03$ & 0.91 & $7.23 \mathrm{e}-01$ & 0.26 & $1.84 \mathrm{e}-05$ & 3.00 \\
& 2 & $4.81 \mathrm{e}-03$ & 0.93 & $6.47 \mathrm{e}-01$ & 0.27 & $2.70 \mathrm{e}-05$ & 2.00 \\
& 3 & $2.47 \mathrm{e}-03$ & 0.96 & $4.99 \mathrm{e}-01$ & 0.38 & $3.93 \mathrm{e}-05$ & 1.00 \\
\hline \multirow{5}{*}{ NLMPFA } & 0 & $8.20 \mathrm{e}-03$ & - & $3.73 \mathrm{e}-01$ & - & $4.00 \mathrm{e}-13$ & 4.00 \\
& 1 & $6.25 \mathrm{e}-03$ & 0.95 & $3.06 \mathrm{e}-01$ & 0.69 & $4.00 \mathrm{e}-13$ & 3.00 \\
& 2 & $4.23 \mathrm{e}-03$ & 0.96 & $2.27 \mathrm{e}-01$ & 0.73 & $4.00 \mathrm{e}-13$ & 2.00 \\
& 3 & $2.12 \mathrm{e}-03$ & 1.00 & $1.31 \mathrm{e}-01$ & 0.80 & $2.00 \mathrm{e}-13$ & 1.00 \\
\hline \multirow{5}{*}{ AvgMPFA } & 0 & $9.10 \mathrm{e}-03$ & - & $7.82 \mathrm{e}-01$ & - & $1.07 \mathrm{e}-05$ & 4.00 \\
& 1 & $7.01 \mathrm{e}-03$ & 0.91 & $7.24 \mathrm{e}-01$ & 0.26 & $1.81 \mathrm{e}-05$ & 3.00 \\
& 2 & $4.81 \mathrm{e}-03$ & 0.93 & $6.48 \mathrm{e}-01$ & 0.27 & $2.66 \mathrm{e}-05$ & 2.00 \\
& 3 & $2.47 \mathrm{e}-03$ & 0.96 & $5.00 \mathrm{e}-01$ & 0.38 & $3.89 \mathrm{e}-05$ & 1.00
\end{tabular}

Table 4 Discrete space-time error norms (scaled by the factor $\frac{1}{\psi(1)}$ ) and convergence rates $(c r)$ with respect to temporal discretization for quasi one-dimensional test case (situation (a) in Figure 2 for the variable $\psi(u)$.

\begin{tabular}{c|c|cccccc} 
scheme & $m$ & $\frac{1}{\psi(1)}\left\|\psi_{m}-\psi_{\text {ref }}\right\|_{L^{2}}$ & $c r$ & $\frac{1}{\psi(1)}\left\|\psi_{m}-\psi_{\text {ref }}\right\|_{\mathscr{T}}$ & $c r$ & $\frac{1}{\alpha_{0}} e_{\mathcal{T}, m}$ & nIt \\
\hline \multirow{3}{*}{ NLTPFA } & 0 & $7.13 \mathrm{e}-03$ & - & $1.29 \mathrm{e}-01$ & - & $3.63 \mathrm{e}-01$ & 864 \\
& 1 & $5.34 \mathrm{e}-03$ & 1.01 & $1.01 \mathrm{e}-01$ & 0.85 & $3.62 \mathrm{e}-01$ & 1002 \\
& 2 & $3.54 \mathrm{e}-03$ & 1.01 & $7.08 \mathrm{e}-02$ & 0.87 & $3.62 \mathrm{e}-01$ & 1331 \\
& 3 & $1.73 \mathrm{e}-03$ & 1.03 & $3.72 \mathrm{e}-02$ & 0.93 & $3.61 \mathrm{e}-01$ & 2150 \\
\hline \multirow{5}{*}{ NLMPFA } & 0 & $6.95 \mathrm{e}-03$ & - & $1.26 \mathrm{e}-01$ & - & $3.64 \mathrm{e}-01$ & 315 \\
& 1 & $5.20 \mathrm{e}-03$ & 1.01 & $9.85 \mathrm{e}-02$ & 0.85 & $3.63 \mathrm{e}-01$ & 416 \\
& 2 & $3.44 \mathrm{e}-03$ & 1.02 & $6.91 \mathrm{e}-02$ & 0.87 & $3.63 \mathrm{e}-01$ & 604 \\
& 3 & $1.68 \mathrm{e}-03$ & 1.04 & $3.62 \mathrm{e}-02$ & 0.93 & $3.62 \mathrm{e}-01$ & 1091 \\
\hline \multirow{3}{*}{ AvgMPFA } & 0 & $7.13 \mathrm{e}-03$ & - & $1.29 \mathrm{e}-01$ & - & $3.63 \mathrm{e}-01$ & 861 \\
& 1 & $5.34 \mathrm{e}-03$ & 1.01 & $1.01 \mathrm{e}-01$ & 0.85 & $3.62 \mathrm{e}-01$ & 1002 \\
& 2 & $3.54 \mathrm{e}-03$ & 1.01 & $7.08 \mathrm{e}-02$ & 0.87 & $3.62 \mathrm{e}-01$ & 1330 \\
& 3 & $1.73 \mathrm{e}-03$ & 1.03 & $3.72 \mathrm{e}-02$ & 0.93 & $3.61 \mathrm{e}-01$ & 2157
\end{tabular}

\subsubsection{Saturation over- and undershoots}

Here, the dependency of saturation over- and undershoots on the scaling factor $\Xi$ is examined. For this purpose, the Neumann boundary conditions are replaced by Dirichlet conditions (situation (b) in Figure 2 and the simulation is run on the coarsest grid with $\delta t=0.3 \mathrm{~s}$ for different values of $\Xi$. Besides this, the same setup is chosen as described in Section 6.1.1. Choosing the same value of $p$ at the left and right boundaries of the domain results in a constant global pressure, and thus in $\mathbf{v}_{\mathrm{T}}=0$. Therefore, flow is purely driven by the term $-\boldsymbol{\Lambda} \nabla \psi$ (see equation (3a)). This allows to investigate the influence of $\Xi$ on saturation over- and undershoots without having an influence of the term $f_{1} \mathbf{v}_{\mathrm{T}}$.

The maximum saturation overshoots are plotted for different values of $\Xi$ in Figure 4 (left). It is observed that the overshoots produced by the NLTPFA and AvgMPFA schemes scale with $\Xi$. Thus, these overshoots can be reduced by increasing $\Xi$. For the NLMPFA scheme, the overshoots are in the range of the solver tolerances and thus no overshoots are observed. This is in accordance with Proposition 3 . The influence of the scaling factor on the total number of Newton iterations is shown in Figure 4 (right). It can be seen that with increasing $\Xi$, the number of Newton iterations also increases. Please note that $\psi(1)$ is already in the range of $10^{6}$ such that for $\Xi=10^{6}$ numerical roundoff errors (for example for calculating numerical derivatives) play an important role. In general, it may cause numerical difficulties if the values for $\psi$ are ten orders of magnitude higher than those of $u$.

Please also note that no undershoots are observed for the setting described above (i.e. $u_{\text {init }}=1$ and the second phase enters the domain on the left). Therefore, to investigate the influence of $\Xi$ on undershoots, we change the setting such that water infiltrates at the left boundary (i.e. $u=1$ ) and set $u_{\text {init }}=0$ with the corresponding Dirichlet condition $u=0$ at the right boundary. For this setup, undershoots are observed as shown in Figure 5 . It can be seen in this figure that the undershoots of 

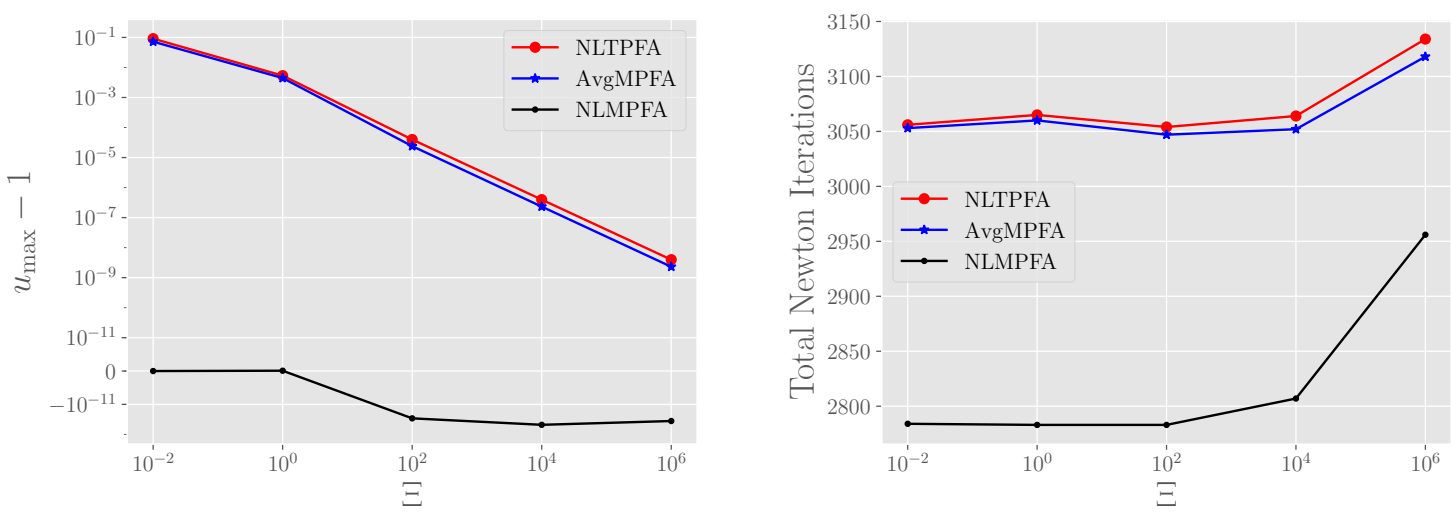

Fig. 4 Saturation overshoots (left) and total Newton iterations (right) plotted for different scaling factors $\Xi$ on the coarsest grid and for a maximum time step size of $\delta t=0.3 \mathrm{~s}$.

the NLTPFA and NLMPFA schemes are in the range of the solver tolerances and thus no undershoots are observed. This is in accordance with Proposition 3 . Again, the undershoots of the AvgMPFA can be reduced by increasing $\Xi$, at least if $\Xi>10^{2}$, which however may influence the Newton convergence behavior as shown in Figure 5 (right). In general, it is beneficial to choose a different scaling factor for $u<0$ than for $u>1$ in $(9)$ because $\psi(1)$ is already in the range of $10^{6}$.

In the following test cases, we set $\Xi=1$ for which one also obtains reasonable results.
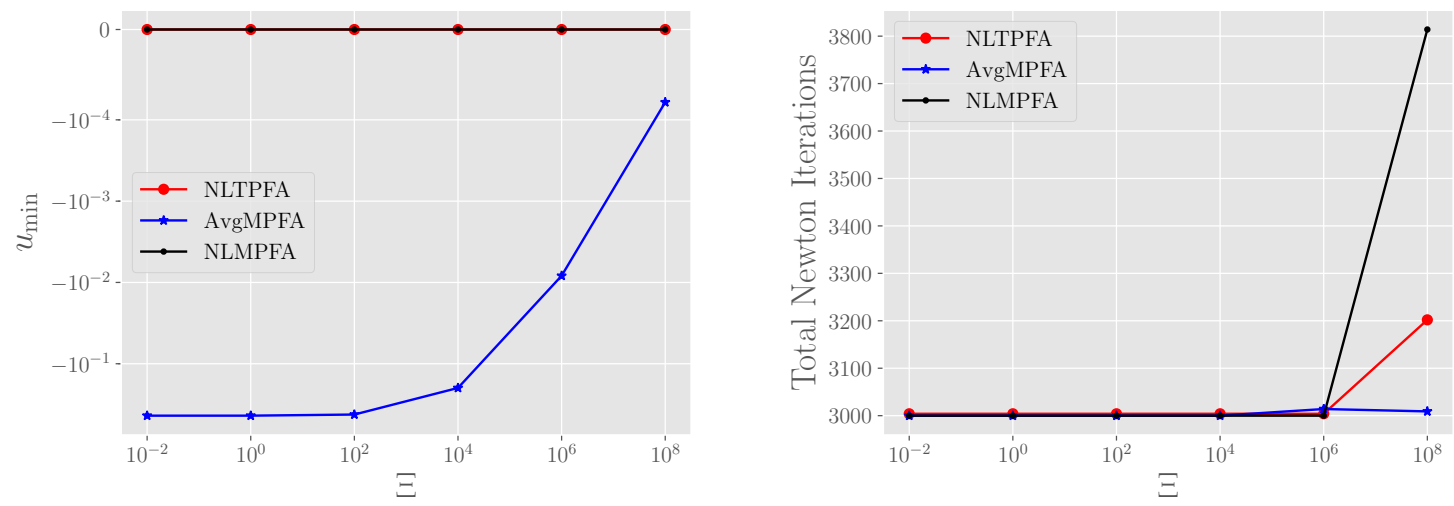

Fig. 5 Saturation undershoots (left) and total Newton iterations (right) plotted for different scaling factors $\Xi$ on the coarsest grid and for a maximum time step size of $\delta t=0.3 \mathrm{~s}$.

\subsection{Lenses test case}

In the following test case, we investigate a two-dimensional setup with domain $\Omega=[0,20] \times[0,10]$ (see Figure 6). Again, there are no source or sink terms (i.e. $s^{+}=s^{-}=0$ ) but in contrast to the previous test case gravity is included. The simulation time is $T=3 \cdot 10^{4} \mathrm{~s}$, the porosity is set to $\phi=0.4$, and the permeability is given as $\boldsymbol{\Lambda}=10^{-10} \mathbf{I}$ except for three lenses within the domain $\Omega_{\text {lenses }}=[2,8] \times[4,5] \cup[7,13] \times[6,7] \cup[10,18] \times[2,3]$ (see Figure 6) with a permeability that is

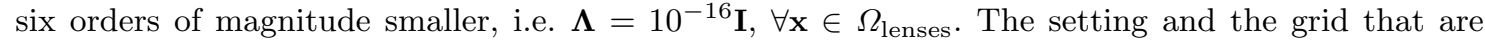
used for this test case are shown in Figure 6. Neumann boundary conditions are set at the top and bottom boundaries, whereas Dirichlet conditions are set on the left and right boundaries. The 


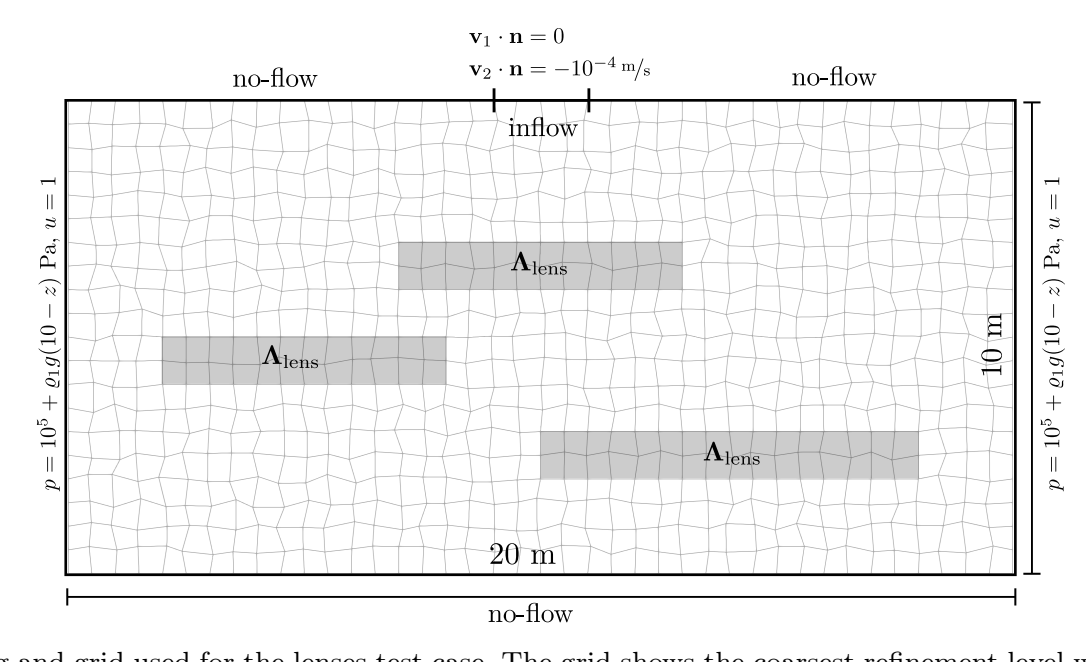

Fig. 6 Setting and grid used for the lenses test case. The grid shows the coarsest refinement level with $40 x 20$ cells.

non-wetting fluid enters the domain at the boundary $\Gamma_{\text {inflow }}=[9,11] \times\{10\}$, with an inflow velocity of $10^{-4} \mathrm{~m} / \mathrm{s}$. Initially, it is assumed that the domain is fully-saturated with the wetting fluid phase, i.e. $u_{\text {init }}(\mathbf{x})=1$.

The densities and the viscosities of the fluids are the same as in the previous test case, i.e. $\varrho_{1}=1000.0 \mathrm{~kg} / \mathrm{m}^{3}, \varrho_{2}=1500.0 \mathrm{~kg} / \mathrm{m}^{3}, \mu_{1}=\mu_{2}=0.003 \mathrm{Pas}$. The mobility functions are given by

$$
\lambda_{1}(u)=\frac{u^{2}}{\mu_{1}}, \quad \lambda_{2}(u)=\frac{1-u^{2}}{\mu_{2}}, \quad \forall u \in[0,1],
$$

Again, formula (104) is used for all $u \notin[0,1]$, which guarantees that (A6) (A7) are fulfilled. For the capillary pressure, a Brooks-Corey-type relationship is used [9]:

$$
p_{c}(u)=10^{4} u^{-\frac{1}{\lambda_{\mathrm{BC}}}}
$$

with $\lambda_{\mathrm{BC}}=1$. For these functions, an analytical expression for $\psi$ can be derived:

$$
\psi(u)=\frac{10^{4}}{\mu_{1}}\left(u-\frac{1}{3} u^{3}\right)
$$

which is used for all $u \in[0,1]$ and the linearization $(9)$ is utilized for all other values of $u$.

For solving the occurring linearized systems of equations, an iterative linear solver is used, namely, a stabilized bi-conjugate gradient (BiCGSTAB) method with an algebraic multigrid preconditioner [5], where the tolerance for the residual reduction is set to $10^{-6}$.

\subsubsection{Spatial convergence}

To investigate the spatial convergence a constant time step size of $\delta t=5 \mathrm{~s}$ is applied and the grid is successively refined, with a maximum refinement level of $m_{\max }=4$.

For this test case, the total velocity is not constant, which is why the convergence rates of $p$ are also investigated. Please also note that, even though $\lambda_{\mathrm{T}}=$ const, the pressure equation 28b depends on $u$ because of the gravity term $\varrho_{f} \mathbf{g}$, with $\varrho_{f}=\varrho_{1} f_{1}+\varrho_{2} f_{2}$. Therefore, the equations 28a) and $28 \mathrm{~b})$ are fully-coupled.

Figure 7 shows the saturation solution of the NLMPFA scheme for the grid with refinement level $m=3$ and the solution differences between two consecutive grid refinement levels (the solution of grid level $m=4$ is mapped to the one of level $m=3$ ). The saturation profile looks as expected, the non-wetting fluid does not flow through the impermeable lenses but instead flows round these 
lenses and accumulates at the bottom boundary because of the given no-flow condition. The flow is buoyancy driven and caused by the density differences. The results of the NLTPFA and AvgMPFA schemes look almost the same and are therefore not shown. As before, it can be seen that the regions of high saturation errors and the regions of high $\psi$ errors are not the same (caused by the fact that $\psi(1)=0)$. The largest differences of $p$ are at the boundary or at the lenses.
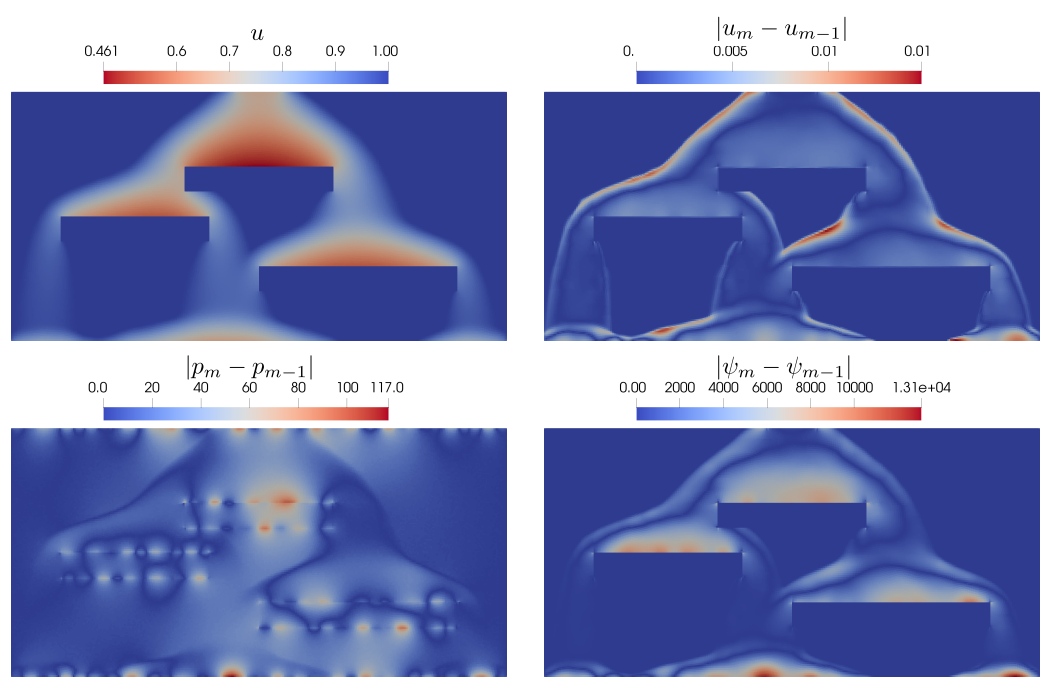

Fig. 7 Saturation solution of NLMPFA scheme and difference between fine solution $\left(u_{m}, \psi_{m}, p_{m}\right)$ and coarser solution $\left(u_{m-1}, \psi_{m-1}, p_{m-1}\right)$ (mapping is done using formula $103 \mathrm{p}$ ) for $m=4$. The results are shown for the lenses test case.

Table 5 Discrete error norms and convergence rates $(c r)$ with respect to spatial discretization for lenses test case for the variable $u$.

\begin{tabular}{c|c|cccccc} 
scheme & $m$ & $\frac{\left\|u_{m}-u_{m-1}\right\|_{L^{2}}}{\sqrt{\Omega}}$ & $c r$ & $\frac{\left\|u_{m}-u_{m-1}\right\|_{\mathscr{T}}}{\sqrt{\Omega}}$ & $c r$ & $u_{\max }-1$ & $h_{\mathcal{D}}$ \\
\hline \multirow{3}{*}{ NLTPFA } & 1 & 1.04 & - & 2.35 & - & $3.69 \mathrm{e}-7$ & $9.15 \mathrm{e}-1$ \\
& 2 & $6.59 \mathrm{e}-1$ & 0.77 & 2.17 & 0.14 & $5.16 \mathrm{e}-5$ & $5.09 \mathrm{e}-1$ \\
& 3 & $4.00 \mathrm{e}-1$ & 0.78 & 1.91 & 0.20 & $3.89 \mathrm{e}-5$ & $2.68 \mathrm{e}-1$ \\
& 4 & $2.31 \mathrm{e}-1$ & 0.82 & 1.61 & 0.25 & $1.45 \mathrm{e}-5$ & $1.38 \mathrm{e}-1$ \\
\hline \multirow{3}{*}{ NLMPFA } & 1 & 1.03 & - & 2.32 & - & 0.00 & $9.15 \mathrm{e}-1$ \\
& 2 & $6.64 \mathrm{e}-1$ & 0.75 & 2.17 & 0.12 & 0.00 & $5.09 \mathrm{e}-1$ \\
& 3 & $4.05 \mathrm{e}-1$ & 0.77 & 1.91 & 0.20 & $9.99 \mathrm{e}-14$ & $2.68 \mathrm{e}-1$ \\
& 4 & $2.34 \mathrm{e}-1$ & 0.82 & 1.61 & 0.25 & $9.99 \mathrm{e}-14$ & $1.38 \mathrm{e}-1$ \\
\hline & 1 & $9.99 \mathrm{e}-1$ & - & 2.25 & - & $9.43 \mathrm{e}-7$ & $9.15 \mathrm{e}-1$ \\
AvgMPFA & 2 & $6.41 \mathrm{e}-1$ & 0.76 & 2.10 & 0.12 & $5.20 \mathrm{e}-5$ & $5.09 \mathrm{e}-1$ \\
& 3 & $3.87 \mathrm{e}-1$ & 0.79 & 1.84 & 0.20 & $4.45 \mathrm{e}-5$ & $2.68 \mathrm{e}-1$ \\
& 4 & $2.22 \mathrm{e}-1$ & 0.83 & 1.55 & 0.26 & $1.46 \mathrm{e}-5$ & $1.38 \mathrm{e}-1$
\end{tabular}

The convergence rates are shown in Table 5.7. Furthermore, saturation overshoots are shown in Table 5, the total number of Newton iterations together with the coercivity estimates in Table 6. and the minimum total pressure value $p_{\min }$ in Table 7. The convergence rates are quite similar for all schemes. The highest rates are observed for the variable $p$, for which all schemes converge with approximately first order with respect to both discrete norms. For the variable $u$ all schemes convergence with a rate of approximately 0.8 for the discrete $L^{2}(\Omega \times(0, T))$-norm and with a rate of approximately $0.2-0.25$ with respect to the discrete $L^{2}\left(0, T ; H^{1}(\Omega)\right)$-norm. Again, the only scheme that does not produce saturation overshoots is the NLMPFA scheme, where the overshoots are below $1 \mathrm{e}-13$ and thus negligible. For $\psi$ the rates are slightly higher and again quite similar for all schemes. 
Table 6 Discrete error norms and convergence rates $(c r)$ with respect to spatial discretization for lenses test case for the variable $\psi(u)$.

\begin{tabular}{c|c|cccccc}
\multirow{2}{*}{ scheme } & $m$ & $\frac{\left\|\psi_{m}-\psi_{m-1}\right\|_{L}}{\sqrt{\Omega} \psi(1)}$ & $c r$ & $\frac{\left\|\psi_{m}-\psi_{m-1}\right\| \mathscr{T}}{\sqrt{\Omega} \psi(1)}$ & \multirow{2}{*}{$c r$} & $\frac{1}{\alpha_{0}} e_{\mathcal{T}, m}$ & nIt \\
\hline \multirow{3}{*}{ NLTPFA } & 1 & $5.59 \mathrm{e}-1$ & - & 1.21 & - & $2.23 \mathrm{e}+5$ & 12128 \\
& 2 & $3.42 \mathrm{e}-1$ & 0.84 & 1.03 & 0.28 & $1.30 \mathrm{e}+5$ & 12793 \\
& 3 & $1.97 \mathrm{e}-1$ & 0.87 & $8.01 \mathrm{e}-1$ & 0.39 & $7.05 \mathrm{e}+4$ & 16249 \\
& 4 & $1.07 \mathrm{e}-1$ & 0.91 & $5.90 \mathrm{e}-1$ & 0.46 & $3.70 \mathrm{e}+4$ & 18007 \\
\hline \multirow{3}{*}{ NLMPFA } & 1 & $5.56 \mathrm{e}-1$ & - & 1.21 & - & $2.23 \mathrm{e}+5$ & 12231 \\
& 2 & $3.46 \mathrm{e}-1$ & 0.81 & 1.04 & 0.26 & $1.30 \mathrm{e}+5$ & 13124 \\
& 3 & $1.99 \mathrm{e}-1$ & 0.86 & $8.09 \mathrm{e}-1$ & 0.39 & $7.06 \mathrm{e}+4$ & 16522 \\
& 4 & $1.08 \mathrm{e}-1$ & 0.92 & $5.94 \mathrm{e}-1$ & 0.46 & $3.70 \mathrm{e}+4$ & 18015 \\
\hline \multirow{3}{*}{ AvgMPFA } & 1 & $5.12 \mathrm{e}-1$ & - & 1.13 & - & $2.20 \mathrm{e}+5$ & 12128 \\
& 2 & $3.20 \mathrm{e}-1$ & 0.80 & $9.79 \mathrm{e}-1$ & 0.25 & $1.28 \mathrm{e}+5$ & 12805 \\
& 3 & $1.83 \mathrm{e}-1$ & 0.87 & $7.64 \mathrm{e}-1$ & 0.39 & $7.01 \mathrm{e}+4$ & 16216 \\
& 4 & $9.89 \mathrm{e}-2$ & 0.92 & $5.60 \mathrm{e}-1$ & 0.46 & $3.68 \mathrm{e}+4$ & 18008
\end{tabular}

Table 7 Discrete error norms and convergence rates $(c r)$ with respect to spatial discretization for lenses test case for the variable $p$.

\begin{tabular}{c|c|cccccc}
\multirow{2}{*}{ scheme } & $m$ & $\frac{\left\|p_{m}-p_{m-1}\right\|_{L^{2}}}{\sqrt{\Omega} 10^{5}}$ & $c r$ & $\frac{\left\|p_{m}-p_{m-1}\right\|_{\mathscr{T}}}{\sqrt{\Omega} 10^{5}}$ & $c r$ & $\frac{1}{\alpha_{0}} e_{\mathcal{T}, m}$ & $p_{\min }$ \\
\hline \multirow{3}{*}{ NLTPFA } & 1 & $2.59 \mathrm{e}-1$ & - & $5.45 \mathrm{e}-1$ & - & $8.43 \mathrm{e}+5$ & $1.02 \mathrm{e}+5$ \\
& 2 & $1.23 \mathrm{e}-1$ & 1.27 & $2.65 \mathrm{e}-1$ & 1.23 & $8.73 \mathrm{e}+5$ & $1.01 \mathrm{e}+5$ \\
& 3 & $6.20 \mathrm{e}-2$ & 1.07 & $1.33 \mathrm{e}-1$ & 1.08 & $8.85 \mathrm{e}+5$ & $1.01 \mathrm{e}+5$ \\
& 4 & $3.17 \mathrm{e}-2$ & 1.01 & $6.97 \mathrm{e}-2$ & 0.96 & $8.91 \mathrm{e}+5$ & $1.00 \mathrm{e}+5$ \\
\hline \multirow{3}{*}{ NLMPFA } & 1 & $2.60 \mathrm{e}-1$ & - & $5.39 \mathrm{e}-1$ & - & $8.43 \mathrm{e}+5$ & $1.02 \mathrm{e}+5$ \\
& 2 & $1.23 \mathrm{e}-1$ & 1.27 & $2.62 \mathrm{e}-1$ & 1.23 & $8.73 \mathrm{e}+5$ & $1.01 \mathrm{e}+5$ \\
& 3 & $6.25 \mathrm{e}-2$ & 1.06 & $1.32 \mathrm{e}-1$ & 1.07 & $8.85 \mathrm{e}+5$ & $1.01 \mathrm{e}+5$ \\
& 4 & $3.20 \mathrm{e}-2$ & 1.00 & $6.97 \mathrm{e}-2$ & 0.96 & $8.91 \mathrm{e}+5$ & $1.00 \mathrm{e}+5$ \\
\hline \multirow{3}{*}{ AvgMPFA } & 1 & $1.35 \mathrm{e}-1$ & - & $2.98 \mathrm{e}-1$ & - & $8.44 \mathrm{e}+5$ & $1.02 \mathrm{e}+5$ \\
& 2 & $6.67 \mathrm{e}-2$ & 1.20 & $1.50 \mathrm{e}-1$ & 1.17 & $8.73 \mathrm{e}+5$ & $1.01 \mathrm{e}+5$ \\
& 3 & $3.37 \mathrm{e}-2$ & 1.07 & $7.64 \mathrm{e}-2$ & 1.05 & $8.85 \mathrm{e}+5$ & $1.01 \mathrm{e}+5$ \\
& 4 & $1.72 \mathrm{e}-2$ & 1.01 & $4.12 \mathrm{e}-2$ & 0.93 & $8.91 \mathrm{e}+5$ & $1.00 \mathrm{e}+5$
\end{tabular}

A possible reason for these slightly higher rates is the fact that the highest saturation errors are located at regions where $u$ is close to one, for which $\psi$ is quite small (see Figure 7). Looking at the total number of Newton iterations it can also be seen that all schemes behave similar, in contrast to the previous test case. Furthermore, the coercivity estimates for $p$ indicate that the schemes are coercive. However, the estimates for $\psi$ decrease with increasing mesh refinement and therefore it is not clear if these estimates are bounded from below.

\subsubsection{Temporal convergence}

To examine the temporal convergence rates, a fine grid resolution (160x320 cells) is chosen and a reference solution is calculated by using a small time step size of $\delta t_{\text {ref }}=5 \mathrm{~s}$. The results are listed in Table 8, 10. For all variables a first order convergence is observed with respect to the discrete $L^{2}(\Omega \times(0, T))$-norm, the rates with respect to the discrete $L^{2}\left(0, T ; H^{1}(\Omega)\right)$-norm are slightly lower. The behavior in terms of saturation overshoots and Newton convergence are similar than for the spatial convergence in the previous section. Furthermore, the coercivity estimates seem to be bounded from below.

\section{Conclusion}

In this work, we have presented the mathematical analysis of the incompressible immiscible twophase flow equations and performed different numerical test cases to verify the theoretical findings. The mathematical formulation of the two-phase flow problem together with some assumptions on the occurring nonlinear functions, used to perform the proof of convergence, have been presented in 
Table 8 Discrete error norms and convergence rates $(c r)$ with respect to temporal discretization for lenses test case for the variable $u$.

\begin{tabular}{c|c|cccccc} 
scheme & $m$ & $\frac{\left\|u_{m}-u_{\text {ref }}\right\|_{L}}{\sqrt{\Omega}}$ & $c r$ & $\frac{\left\|u_{m}-u_{\text {ref }}\right\|_{\mathscr{T}}}{\sqrt{\Omega}}$ & $c r$ & $u_{\text {max }}-1$ & $\delta t$ \\
\hline \multirow{3}{*}{ NLTPFA } & 0 & $4.62 \mathrm{e}-1$ & - & 3.62 & - & $1.46 \mathrm{e}-5$ & 400.00 \\
& 1 & $3.60 \mathrm{e}-1$ & 0.87 & 2.90 & 0.76 & $1.50 \mathrm{e}-5$ & 300.00 \\
& 2 & $2.51 \mathrm{e}-1$ & 0.89 & 2.10 & 0.80 & $1.50 \mathrm{e}-5$ & 200.00 \\
& 3 & $1.31 \mathrm{e}-1$ & 0.94 & 1.14 & 0.87 & $1.48 \mathrm{e}-5$ & 100.00 \\
\hline \multirow{5}{*}{ NLMPFA } & 0 & $4.61 \mathrm{e}-1$ & - & 3.61 & - & $1.17 \mathrm{e}-11$ & 400.00 \\
& 1 & $3.60 \mathrm{e}-1$ & 0.87 & 2.90 & 0.76 & $1.34 \mathrm{e}-11$ & 300.00 \\
& 2 & $2.50 \mathrm{e}-1$ & 0.89 & 2.09 & 0.80 & $9.80 \mathrm{e}-12$ & 200.00 \\
& 3 & $1.31 \mathrm{e}-1$ & 0.94 & 1.14 & 0.87 & $8.60 \mathrm{e}-12$ & 100.00 \\
\hline \multirow{3}{*}{ AvgMPFA } & 0 & $4.61 \mathrm{e}-1$ & - & 3.62 & - & $1.43 \mathrm{e}-5$ & 400.00 \\
& 1 & $3.59 \mathrm{e}-1$ & 0.87 & 2.91 & 0.76 & $1.42 \mathrm{e}-5$ & 300.00 \\
& 2 & $2.50 \mathrm{e}-1$ & 0.89 & 2.10 & 0.81 & $1.42 \mathrm{e}-5$ & 200.00 \\
& 3 & $1.30 \mathrm{e}-1$ & 0.94 & 1.15 & 0.87 & $1.40 \mathrm{e}-5$ & 100.00
\end{tabular}

Table 9 Discrete error norms and convergence rates $(c r)$ with respect to temporal discretization for lenses test case for the variable $\psi(u)$.

\begin{tabular}{|c|c|c|c|c|c|c|c|}
\hline scheme & $m$ & $\frac{\left\|\psi_{m}-\psi_{\text {ref }}\right\|_{L^{2}}}{\sqrt{\Omega} \psi(1)}$ & $\mathrm{cr}$ & $\frac{\left\|\psi_{m}-\psi_{\text {ref }}\right\| \mathscr{T}}{\sqrt{\Omega} \psi(1)}$ & $\mathrm{cr}$ & $\frac{1}{\alpha_{0}} e_{\mathcal{T}, m}$ & nIt \\
\hline \multirow{4}{*}{ NLTPFA } & 0 & $1.37 \mathrm{e}-1$ & - & $8.30 \mathrm{e}-1$ & - & $3.78 \mathrm{e}+4$ & 469 \\
\hline & 1 & $1.04 \mathrm{e}-1$ & 0.95 & $6.43 \mathrm{e}-1$ & 0.88 & $3.76 \mathrm{e}+4$ & 573 \\
\hline & 2 & $7.05 \mathrm{e}-2$ & 0.97 & $4.44 \mathrm{e}-1$ & 0.91 & $3.74 \mathrm{e}+4$ & 752 \\
\hline & 3 & $3.53 \mathrm{e}-2$ & 1.00 & $2.28 \mathrm{e}-1$ & 0.96 & $3.72 \mathrm{e}+4$ & 1259 \\
\hline \multirow{4}{*}{ NLMPFA } & 0 & $1.37 \mathrm{e}-1$ & - & $8.29 \mathrm{e}-1$ & - & $3.78 \mathrm{e}+4$ & 379 \\
\hline & 1 & $1.04 \mathrm{e}-1$ & 0.95 & $6.43 \mathrm{e}-1$ & 0.88 & $3.76 \mathrm{e}+4$ & 486 \\
\hline & 2 & $7.05 \mathrm{e}-2$ & 0.97 & $4.44 \mathrm{e}-1$ & 0.91 & $3.74 \mathrm{e}+4$ & 654 \\
\hline & 3 & $3.53 \mathrm{e}-2$ & 1.00 & $2.28 \mathrm{e}-1$ & 0.96 & $3.72 \mathrm{e}+4$ & 1203 \\
\hline \multirow{4}{*}{ AvgMPFA } & 0 & $1.36 \mathrm{e}-1$ & - & $8.28 \mathrm{e}-1$ & - & $3.77 \mathrm{e}+4$ & 463 \\
\hline & 1 & $1.04 \mathrm{e}-1$ & 0.95 & $6.42 \mathrm{e}-1$ & 0.88 & $3.75 \mathrm{e}+4$ & 583 \\
\hline & 2 & $7.01 \mathrm{e}-2$ & 0.97 & $4.44 \mathrm{e}-1$ & 0.91 & $3.73 \mathrm{e}+4$ & 752 \\
\hline & 3 & $3.51 \mathrm{e}-2$ & 1.00 & $2.28 \mathrm{e}-1$ & 0.96 & $3.70 \mathrm{e}+4$ & 1278 \\
\hline
\end{tabular}

Table 10 Discrete error norms and convergence rates $(c r)$ with respect to temporal discretization for lenses test case for the variable $p$.

\begin{tabular}{c|c|cccccc} 
scheme & $m$ & $\frac{\left\|p_{m}-p_{\text {ref }}\right\|_{L}}{\sqrt{\Omega} 10^{5}}$ & $c r$ & $\frac{\left\|p_{m}-p_{\text {ref }}\right\| \mathscr{T}}{\sqrt{\Omega} 10^{5}}$ & $c r$ & $\frac{1}{\alpha_{0}} e_{\mathcal{T}, m}$ & $p_{\min }$ \\
\hline \multirow{3}{*}{ NLTPFA } & 0 & $2.12 \mathrm{e}-2$ & - & $4.09 \mathrm{e}-2$ & - & $8.91 \mathrm{e}+5$ & $1.00 \mathrm{e}+5$ \\
& 1 & $1.62 \mathrm{e}-2$ & 0.94 & $3.19 \mathrm{e}-2$ & 0.87 & $8.91 \mathrm{e}+5$ & $1.00 \mathrm{e}+5$ \\
& 2 & $1.10 \mathrm{e}-2$ & 0.96 & $2.22 \mathrm{e}-2$ & 0.89 & $8.91 \mathrm{e}+5$ & $1.00 \mathrm{e}+5$ \\
& 3 & $5.53 \mathrm{e}-3$ & 0.99 & $1.16 \mathrm{e}-2$ & 0.94 & $8.91 \mathrm{e}+5$ & $1.00 \mathrm{e}+5$ \\
\hline \multirow{3}{*}{ NLMPFA } & 0 & $2.12 \mathrm{e}-2$ & - & $4.08 \mathrm{e}-2$ & - & $8.91 \mathrm{e}+5$ & $1.00 \mathrm{e}+5$ \\
& 1 & $1.62 \mathrm{e}-2$ & 0.94 & $3.18 \mathrm{e}-2$ & 0.87 & $8.91 \mathrm{e}+5$ & $1.00 \mathrm{e}+5$ \\
& 2 & $1.10 \mathrm{e}-2$ & 0.96 & $2.21 \mathrm{e}-2$ & 0.89 & $8.91 \mathrm{e}+5$ & $1.00 \mathrm{e}+5$ \\
& 3 & $5.53 \mathrm{e}-3$ & 0.99 & $1.15 \mathrm{e}-2$ & 0.94 & $8.91 \mathrm{e}+5$ & $1.00 \mathrm{e}+5$ \\
\hline \multirow{5}{*}{ AvgMPFA } & 0 & $2.10 \mathrm{e}-2$ & - & $4.08 \mathrm{e}-2$ & - & $8.91 \mathrm{e}+5$ & $1.00 \mathrm{e}+5$ \\
& 1 & $1.61 \mathrm{e}-2$ & 0.94 & $3.18 \mathrm{e}-2$ & 0.87 & $8.91 \mathrm{e}+5$ & $1.00 \mathrm{e}+5$ \\
& 2 & $1.09 \mathrm{e}-2$ & 0.96 & $2.21 \mathrm{e}-2$ & 0.90 & $8.91 \mathrm{e}+5$ & $1.00 \mathrm{e}+5$ \\
& 3 & $5.47 \mathrm{e}-3$ & 0.99 & $1.15 \mathrm{e}-2$ & 0.94 & $8.91 \mathrm{e}+5$ & $1.00 \mathrm{e}+5$
\end{tabular}

Section 2. In Sections 3 and 4, a general finite volume discretization framework has been introduced and the proof of convergence has been given. In contrast to existing literature, this general framework also includes so-called nonlinear flux discretization schemes. Two representatives of such schemes, namely the NLTPFA and NLMPFA schemes, have then been presented in Section 5 where we have also shown some fundamental properties of these schemes. Finally, these schemes have been numerically investigated in Section 6 for a quasi one-dimensional test case and for a more complicated test case, which includes gravity effects and heterogeneities. For these test cases, the convergence with respect to a discrete $L^{2}(\Omega \times(0, T))$ - and $L^{2}\left(0, T ; H^{1}(\Omega)\right)$-norm has been studied. Furthermore, it has been demonstrated that the NLMPFA scheme does not produce any saturation over- or undershoots, whereas the NLTPFA scheme is positivity-preserving. This is generally not given for linear schemes, 
which may produce over- and undershoots. In future work, these results will also be compared to other discretization schemes and the theory will be extended to more general problems, for example by allowing compressibility effects.

\section{Acknowledgements}

The authors Bernd Flemisch and Martin Schneider would like to thank the German Research Foundation (DFG, Deutsche Forschungsgemeinschaft) for supporting this work by funding SFB 1313, Project Number 327154368.

\section{Appendix}

\subsection{Weak consistency}

In this section, the weak consistency in $L^{2}\left(0, T ; H_{0}^{1}(\Omega)\right)$ is proven in Proposition 4 . To show this result, we first establish Lemma 10 and 11 also used in other proofs.

Lemma 10 (Discrete $H^{1}$-norm controlled by $H^{1}$-norm) Let $\mathcal{D}$ be an admissible space discretization matching Definition 2. Then, there exists a constant $C_{16}>0$ depending on $\alpha, d, \zeta_{1}$ and $\zeta_{3}$ s.t., for all $v \in H_{0}^{1}(\Omega)$,

$$
\left\|v_{\mathcal{T}}\right\|_{\mathcal{T}} \leq C_{16}\|\nabla v\|_{\left[L^{2}(\Omega)\right]^{d}} .
$$

Proof Using the definition of $v_{\mathcal{T}}$ from Section 3.2.2 and Definition 21 of $\gamma_{\sigma} v_{\mathcal{T}}$, we get

$$
\frac{\left(\gamma_{\sigma} v_{\mathcal{T}}-v_{K}\right)^{2}}{d_{K, \sigma}}+\frac{\left(\gamma_{\sigma} v_{\mathcal{T}}-v_{L}\right)^{2}}{d_{L, \sigma}}=\min _{v_{\sigma} \in \mathbb{R}}\left(\frac{\left(v_{\sigma}-v_{K}\right)^{2}}{d_{K, \sigma}}+\frac{\left(v_{\sigma}-v_{L}\right)^{2}}{d_{L, \sigma}}\right)
$$

for all $\sigma \in \mathcal{E}_{\text {int }}, \mathcal{T}_{\sigma}=\{K, L\}$. Then, choosing, for all $\sigma \in \mathcal{E}, v_{\sigma}=\langle v\rangle_{\sigma}$, we obtain

$$
\left\|v_{\mathcal{T}}\right\|_{\mathcal{T}}^{2} \leq \sum_{K \in \mathcal{T}} \sum_{\sigma \in \mathcal{E}_{K}}|\sigma| \frac{\left(v_{\sigma}-v_{K}\right)^{2}}{d_{K, \sigma}}
$$

Using Lemma 6.6 of [15], we get

$$
\left\|v_{\mathcal{T}}\right\|_{\mathcal{T}}^{2} \leq \sum_{K \in \mathcal{T}} \sum_{\sigma \in \mathcal{E}_{K}} C \frac{\operatorname{diam}(K)}{d_{K, \sigma}} \int_{K}|\nabla v|^{2} \mathrm{~d} x,
$$

where the constant $C$ only depends on $\alpha$ and $d$. Then, thanks to (17)- 18, the result is obtained with $C_{16}=\sqrt{\frac{\zeta_{1}}{\zeta_{3}} C}$.

Lemma 11 Let $\mathscr{D}$ be an admissible space-time discretization matching Definition 3 . Then there exists a constant $C_{17}>0$ s.t., for all $v \in L^{2}\left(0, T ; H_{0}^{1}(\Omega)\right)$,

$$
\left\|v_{\mathscr{T}}\right\| \mathscr{T} \leq C_{17}\|v\|_{L^{2}\left(0, T ; H_{0}^{1}(\Omega)\right)} .
$$

Proof Let us consider $v_{\mathscr{T}} \in H_{\mathscr{T}}(\Omega \times(0, T))$ s.t. $v^{(n)}=\frac{1}{\delta t^{\left(n+\frac{1}{2}\right)}} \int_{t^{(n)}}^{t^{(n+1)}} v_{\mathcal{T}} d t$ and $v_{\mathcal{T}}(t) \in H_{\mathcal{T}}(\Omega)$ s.t., for all $K \in \mathcal{T}, v(t)_{\mid K}=\langle v(t)\rangle_{K}$. Then, applying Lemma 10 to $v^{(n)}$ yields

$$
\left\|v_{\mathscr{T}}^{(n)}\right\|_{\mathcal{T}} \leq C_{16}\left\|\nabla v^{(n)}\right\|_{\left[L^{2}(\Omega)\right]^{d}} .
$$

On the other hand, Cauchy-Schwarz inequality leads to

$$
\left\|\nabla v^{(n)}\right\|_{\left[L^{2}(\Omega)\right]^{d}}^{2} \leq \frac{1}{\delta t^{\left(n+\frac{1}{2}\right)}} \int_{\Omega}\left(\int_{t^{(n)}}^{t^{(n+1)}}|\nabla v(x, t)|^{2} d t\right) d x .
$$


Therefore, from the two previous inequalities, it follows that, for all $n \in \llbracket 0, N \rrbracket$,

$$
\left\|v_{\mathscr{T}}^{(n)}\right\|_{\mathcal{T}}^{2} \leq C_{16}^{2} \frac{1}{\delta t^{\left(n+\frac{1}{2}\right)}} \int_{t^{(n)}}^{t^{(n+1)}} \int_{\Omega}|\nabla v(x, t)|^{2} d x d t .
$$

Multiplying the former inequality by $\delta t^{\left(n+\frac{1}{2}\right)}$ and summing it over $n=0, \ldots, N$ yield

$$
\left\|v_{\mathscr{T}}\right\|_{\mathscr{T}}^{2} \leq C_{16}^{2}\|\nabla v\|_{L^{2}((0, T) \times \Omega)}^{2} .
$$

We now state and prove Proposition 4 .

Proposition 4 Assuming properties (P1), (P2) and (P4), for any bounded function $\chi$, then $a_{\mathcal{T}_{m}, \chi,}$, is also weakly consistent on $L^{2}\left(0, T ; H_{0}^{1}(\Omega)\right)$.

Proof Let $\varphi \in L^{2}(0, T ; \mathfrak{D})$ and $q \in L^{2}\left(0, T ; H_{0}^{1}(\Omega)\right)$. Under property $(\mathrm{P} 1)$ the form $a$ is linear with respect to its second argument, we then have, for all $(u, v, w) \in \mathscr{P}^{m}$,

$$
\begin{aligned}
\mid \int_{0}^{T} a_{\mathcal{T}_{m}, \chi, v(t)} & \left(u(t), q_{\mathscr{T}_{m}}(t), w(t)\right) d t-\int_{0}^{T} \int_{\Omega} \chi(v) \Lambda \nabla q \cdot \widetilde{\nabla}_{\mathcal{D}_{m}, \Upsilon_{m}} w d x d t \mid \\
\leq & \left|\int_{0}^{T} a_{\mathcal{T}_{m}, \chi, v(t)}\left(u(t), q_{\mathscr{T}_{m}}(t)-\varphi_{\mathscr{T}_{m}}(t), w(t)\right) d t\right| \\
& +\left|\int_{0}^{T} a_{\mathcal{T}_{m}, \chi, v(t)}\left(u(t), \varphi_{\mathscr{T}_{m}}(t), w(t)\right)-\int_{0}^{T} \int_{\Omega} \chi(v) \Lambda \nabla \varphi \cdot \widetilde{\nabla}_{\mathcal{D}_{m}, \Upsilon_{m}} w d x d t\right| \\
& +\left|\int_{0}^{T} \int_{\Omega} \chi(v) \Lambda \nabla(\varphi-q) \cdot \widetilde{\nabla}_{\mathcal{D}_{m}, \Upsilon_{m}} w d x d t\right| .
\end{aligned}
$$

Using the continuity property (P2) of the bilinear form $a_{\mathcal{T}_{m}, \chi, v(t)}(u(t), . .$.$) , the Cauchy-Schwarz$ inequality for the time integral, and Lemma 11 lead to

$$
\left|\int_{0}^{T} a_{\mathcal{T}_{m}, \chi, v(t)}\left(u(t), q_{\mathscr{T}_{m}}(t)-\varphi_{\mathscr{T}_{m}}(t), w(t)\right) d t\right| \leq C_{\chi}\|q-\varphi\|_{L^{2}\left(0, T ; H_{0}^{1}(\Omega)\right)}\|w\| \mathscr{T}_{m} .
$$

Furthermore, since $\left\|\widetilde{\nabla}_{\mathcal{D}_{m}, \Upsilon_{m}} \eta\right\|_{\left[L^{2}(\Omega)\right]^{d}} \leq \sqrt{d}\|\eta\|_{\mathcal{T}_{m}, \Upsilon_{m}} \leq \frac{2 \sqrt{d}}{\zeta_{4}}\|\eta\|_{\mathcal{T}_{m}}$ for all $\eta \in H_{\mathcal{T}_{m}}(\Omega)$ (as a consequence of (16), the Cauchy-Schwarz inequality, and the equivalence of norms Lemma 1 in [34), and again applying the Cauchy-Schwarz inequality results in

$$
\left|\int_{0}^{T} \int_{\Omega} \chi(v) \Lambda \nabla(\varphi-q) \cdot \widetilde{\nabla}_{\mathcal{D}_{m}, Y_{m}} w d x d t\right| \leq \bar{C}_{\chi}\|q-\varphi\|_{L^{2}\left(0, T ; H_{0}^{1}(\Omega)\right)}\|w\| \mathscr{T}_{m} .
$$

Therefore, dividing (114) by $\|w\|_{\mathscr{T}_{m}}$, taking the infimum over the set $\mathscr{R}_{\mathcal{E}_{m}}$ and the maximum over the set $\mathscr{P}^{m}$, we deduce

$$
\epsilon_{\mathscr{D}_{m}}(q) \leq \tilde{C}_{\chi}\|q-\varphi\|_{L^{2}\left(0, T ; H_{0}^{1}(\Omega)\right)}+\epsilon_{\mathscr{D}_{m}}(\varphi) .
$$

Taking $\lim \sup \epsilon_{\mathscr{D}_{m}}(q)$ in the inequality just above and using the weak consistency property $\left.(\mathrm{P} 4)\right]$ we get

$$
\limsup _{m \rightarrow \infty} \epsilon_{\mathscr{D}_{m}}(q) \leq \tilde{C}_{\chi}\|q-\varphi\|_{L^{2}\left(0, T ; H_{0}^{1}(\Omega)\right)}
$$

Using the density of $L^{2}(0, T ; \mathfrak{D})$ in $L^{2}\left(0, T ; H_{0}^{1}(\Omega)\right)$, there exists a sequence $\left\{\varphi_{l}\right\}_{l \in \mathbb{N}} \subset L^{2}(0, T ; \mathfrak{D})$, s.t. $\varphi_{l} \rightarrow q$ in $L^{2}\left(0, T ; H_{0}^{1}(\Omega)\right)$, and therefore

$$
\limsup _{m \rightarrow \infty} \epsilon_{\mathscr{D}_{m}}(q)=0
$$


8.2 A technical lemma

Lemma 12 Let $\mathcal{D}$ be a space discretization matching Definition 1 and $M: \mathbb{R} \times \mathbb{R} \mapsto \mathbb{R}$ a bounded function. For all $(u, v) \in\left[H_{\mathcal{T}}(\Omega)\right]^{2}$, there exists a constant $C_{18}$ depending on $\|M\|_{\infty}, \beta_{0}, g$ and $\Omega$ such that

$$
\left|\sum_{K \in \mathcal{T}} \sum_{\sigma \in \mathcal{E}_{K}} M\left(u_{1, \sigma}, u_{2, \sigma}\right) G_{K, \sigma} v_{K}\right| \leq C_{18}\|v\|_{\mathcal{T}},
$$

where $u_{1}, u_{2} \in H_{\Delta}(\Omega)$.

Proof Let us denote by $S$ the left hand side of 1115 . Using $G_{K, \sigma}+G_{L, \sigma}=0$ if $\mathcal{T}_{\sigma}=\{K, L\}, \gamma_{\sigma} v=0$ for all $\sigma \in \mathcal{E}_{\text {ext }}$, equality (16), and the Cauchy-Schwarz inequality to deduce

$$
\begin{aligned}
S & =\left|\sum_{K \in \mathcal{T}} \sum_{\sigma \in \mathcal{E}_{K}} M\left(u_{1, \sigma}, u_{2, \sigma}\right) G_{K, \sigma}\left(v_{K}-\gamma_{\sigma} v\right)\right| \\
& \leq\|M\|_{\infty} \beta_{0}|g| \sqrt{d|\Omega|}\|v\|_{\mathcal{T}} .
\end{aligned}
$$

Lemma 13 Let $\psi \in C(\mathbb{R})$ be a nondecreasing Lipschitz-continuous function with $\psi(0)=0$ and $L_{\psi}$ its Lipschitz constant. Then the function $\Psi$ defined by

$$
\Psi(s)=\int_{0}^{s} \psi(x) \mathrm{d} x, \quad \forall s \in \mathbb{R} .
$$

satisfies the following inequality:

$$
0 \leq \frac{\psi(s)^{2}}{2 L_{\psi}} \leq \Psi(s) \leq L_{\psi} \frac{s^{2}}{2}, \quad \forall s \in \mathbb{R} .
$$

Proof On one hand, for any $s \geq 0$ we have $\Psi(s)=\int_{0}^{s}(\psi(x)-\psi(0)) \mathrm{d} x \leq L_{\psi} \int_{0}^{s} x d x=L_{\psi} \frac{s^{2}}{2}$ and for any $s \leq 0, \Psi(s)=\int_{s}^{0}(\psi(0)-\psi(x)) \mathrm{d} x \leq L_{\psi} \int_{s}^{0}(-x) d x=L_{\psi} \frac{s^{2}}{2}$. Therefore, for all $s \in \mathbb{R}$, $\Psi(s) \leq L_{\psi} \frac{s^{2}}{2}$.

On the other hand, we introduce the functions $\psi_{\epsilon}$ and $\Psi_{\epsilon}$ defined respectively for all $s \in \mathbb{R}$ by $\psi_{\epsilon}(s)=\frac{\Psi(s+\epsilon)-\Psi(s)}{\epsilon}$ and $\Psi_{\epsilon}(s)=\int_{0}^{s} \psi_{\epsilon}(x) d x$ with $\epsilon \in \mathbb{R}^{*}$. Then $\psi_{\epsilon}$ is a $C^{1}$-function with $\psi_{\epsilon}^{\prime}(s)=$ $\frac{\psi(s+\epsilon)-\psi(s)}{\epsilon}$, we thus get $\forall s \in \mathbb{R},\left|\psi_{\epsilon}^{\prime}(s)\right| \leq L_{\psi}$ and $\psi_{\epsilon}^{\prime}(s) \geq 0$. We observe that

$$
\begin{aligned}
& \left|\psi_{\epsilon}(s)-\psi(s)\right|=\left|\frac{\int_{s}^{s+\epsilon}(\psi(x)-\psi(s)) d x}{\epsilon}\right| \leq \frac{L_{\psi}}{|\epsilon|}\left|\int_{s}^{s+\epsilon}\right| x-s|d x|=\frac{L_{\psi}|\epsilon|}{2}, \\
& \left|\Psi_{\epsilon}(s)-\Psi(s)\right|=\left|\int_{0}^{s}\left(\psi_{\epsilon}(x)-\psi(x)\right) d x\right| \leq \frac{L_{\psi}|\epsilon|}{2}|s| .
\end{aligned}
$$

By considering the function $G_{\epsilon}$ defined for all $s \in \mathbb{R}$ by $G_{\epsilon}(s)=\Psi_{\epsilon}(s)-\frac{\psi_{\epsilon}(s)^{2}}{2 L_{\psi}}$, we have $G_{\epsilon}^{\prime}(s)=$ $\psi_{\epsilon}(s)\left(1-\frac{\psi_{\epsilon}^{\prime}(s)}{L_{\psi}}\right)$

Owing to 117), we get $\forall s \in \mathbb{R}, G_{\epsilon}(0) \rightarrow 0$ and $G_{\epsilon}(s) \rightarrow \Psi(s)-\frac{\psi(s)^{2}}{2 L_{\psi}}$ as $\epsilon \rightarrow 0$.

By taking $\epsilon>0$, for any $s \geq 0$ we have $\psi_{\epsilon}(s) \geq \psi_{\epsilon}(0)=\frac{\Psi(\epsilon)}{\epsilon} \geq 0$ and then $G_{\epsilon}^{\prime}(s) \geq 0$, which implies $G_{\epsilon}(s) \geq G_{\epsilon}(0)$ and hence as $\epsilon \rightarrow 0^{+}$we deduce that for any $s \geq 0, \Psi(s)-\frac{\psi(s)^{2}}{2 L_{\psi}} \geq 0$. Let us now take 
$\epsilon<0$, for any $s \leq 0$ we have $\psi_{\epsilon}(s) \leq \psi_{\epsilon}(0)=\frac{\Psi(\epsilon)}{\epsilon} \leq 0$ and then $G_{\epsilon}^{\prime}(s) \leq 0$, we thus deduce that $G_{\epsilon}(s) \geq G_{\epsilon}(0)$ which implies as $\epsilon \rightarrow 0^{-}$that for any $s \leq 0, \Psi(s)-\frac{\psi(s)^{2}}{2 L_{\psi}} \geq 0$.

Then we obtain that for all $s \in \mathbb{R}, \frac{\psi(s)^{2}}{2 L_{\psi}} \leq \Psi(s)$.

\section{References}

1. Agélas, L., Di Pietro, D., Droniou, J.: The G method for heterogeneous anisotropic diffusion on general meshes. M2AN Math. Model. Numer. Anal. 44(4), 597-625 (2010)

2. Agélas, L., Guichard, C., Masson, R.: Convergence of finite volume MPFA O type schemes for heterogeneous anisotropic diffusion problems on general meshes. Int. J. Finite Vol. 7(2), 1-33 (2010)

3. Alkmper, M., Dedner, A., Klfkorn, R., Nolte, M.: The DUNE-ALUGrid Module. Archive of Numerical Software 4(1), 1-28 (2016). DOI 10.11588/ans.2016.1.23252

4. Angelini, O., Brenner, K., Hilhorst, D.: A finite volume method on general meshes for a degenerate parabolic convection-reaction-diffusion equation. Numerische Mathematik 123(2), 219-257 (2013). DOI 10.1007/s00211-012-0485-5

5. Blatt, M., Bastian, P.: The iterative solver template library. Springer (2007)

6. Blatt, M., Burchardt, A., Dedner, A., Engwer, C., Fahlke, J., Flemisch, B., Gersbacher, C., Gräser, C., Gruber, F., Grüninger, C., Kempf, D., Klöfkorn, R., Malkmus, T., Müthing, S., Nolte, M., Piatkowski, M., Sander, O.: The distributed and unified numerics environment, version 2.4. Archive of Numerical Software 4(100), 13-29 (2016)

7. Brenier, Y., Jaffré, J.: Upstream differencing for multiphase flow in reservoir simulation. SIAM Journal on Numerical Analysis 28(3), 685-696 (1991)

8. Brenner, K., Masson, R.: Convergence of a vertex centred discretization of two-phase darcy flows on general meshes. International Journal on Finite Volumes 10, 1-37 (2013)

9. Brooks, R., Corey, A.: Hydraulic properties of porous media. Hydrology Papers, Colorado State University (1964)

10. Chavent, G., Jaffré, J.: Mathematical models and finite elements for reservoir simulation: single phase, multiphase and multicomponent flows through porous media, vol. 17. Elsevier (1986)

11. Chen, Z.: Degenerate Two-Phase Incompressible Flow: I. Existence, Uniqueness and Regularity of a Weak Solution. Journal of Differential Equations 171(2), 203 - 232 (2001). DOI https://doi.org/10.1006/jdeq.2000. 3848

12. Danilov, A., Vassilevski, Y.: A monotone nonlinear finite volume method for diffusion equations on conformal polyhedral meshes. Russian Journal of Numerical Analysis and Mathematical Modelling 24(3), 207-227 (2009)

13. Davis, T.A.: Algorithm 832: UMFPACK V4.3 - an unsymmetric-pattern multifrontal method. ACM Transactions on Mathematical Software (TOMS) 30(2), 196-199 (2004)

14. Droniou, J.: Finite volume schemes for diffusion equations: introduction to and review of modern methods. Math. Models Methods Appl. Sci. 24(8), 1575-1619 (2014)

15. Droniou, J., Eymard, R.: Study of the mixed finite volume method for stokes and navier-stokes equations. Numerical Methods for Partial Differential Equations 25(1), 137-171 (2009)

16. Droniou, J., Eymard, R.: The asymmetric gradient discretisation method. In: C. Cancès, P. Omnes (eds.) Finite Volumes for Complex Applications VIII - Hyperbolic, Elliptic and Parabolic Problems, pp. 311-319. Springer International Publishing (2017)

17. Droniou, J., Eymard, R., Gallouët, T., Guichard, C., Herbin, R.: The Gradient Discretisation Method. Springer (2018)

18. Droniou, J., Eymard, R., Herbin, R.: Gradient schemes: Generic tools for the numerical analysis of diffusion equations. M2AN Math. Model. Numer. Anal. 50(3), 749-781 (2016)

19. Droniou, J., Potier, C.L.: Construction and convergence study of schemes preserving the elliptic local maximum principle. SIAM J. Numer. Anal. 49(2), 459-490 (2011)

20. Eymard, R., Gallouët, T.: Convergence d'un schéma de type éléments finis-volumes finis pour un système formé d'une équation elliptique et d'une équation hyperbolique. ESAIM: Mathematical Modelling and Numerical Analysis 27(7), 843-861 (1993)

21. Eymard, R., Gallouët, T., Herbin, R.: The finite volume method, vol. 7. Handbook of Numerical Analysis (2000)

22. Eymard, R., Gallouët, T., Herbin, R.: Discretization of heterogeneous and anisotropic diffusion problems on general nonconforming meshes SUSHI: a scheme using stabilization and hybrid interfaces. IMA Journal of Numerical Analysis 30, 1009-1043 (2010)

23. Eymard, R., Guichard, C., Herbin, R., Masson, R.: Gradient schemes for two-phase flow in heterogeneous porous media and Richards equation. ZAMM-Journal of Applied Mathematics and Mechanics/Zeitschrift für Angewandte Mathematik und Mechanik 94(7-8), 560-585 (2014)

24. Eymard, R., Herbin, R., Michel, A.: Mathematical study of a petroleum-engineering scheme. ESAIM: Mathematical Modelling and Numerical Analysis 37(6), 937-972 (2003) 
25. Hommel, J., Ackermann, S., Beck, M., Becker, B., Class, H., Fetzer, T., Flemisch, B., Gläser, D., Grüninger, C., Heck, K., Kissinger, A., Koch, T., Schneider, M., Seitz, G., Weishaupt, K.: DuMuX 2.10.0 (2016). DOI 10.5281/zenodo.159007. URL https://doi.org/10.5281/zenodo.159007

26. Kroener, D., Luckhaus, S.: Flow of oil and water in a porous medium. Journal of Differential Equations 55(2), 276 - 288 (1984). DOI https://doi.org/10.1016/0022-0396(84)90084-6

27. Lipnikov, K., Svyatskiy, D., Vassilevski, Y.: Interpolation-free monotone finite volume method for diffusion equations on polygonal meshes. J. Comput. Phys. 228(3), 703-716 (2009)

28. Lipnikov, K., Svyatskiy, D., Vassilevski, Y.: Minimal stencil finite volume scheme with the discrete maximum principle. Russian Journal of Numerical Analysis and Mathematical Modelling 27(4), 369-385 (2012)

29. Michel, A.: A finite volume scheme for two-phase immiscible flow in porous media. SIAM Journal on Numerical Analysis 41(4), 1301-1317 (2003)

30. Potier, C.L.: Schéma volumes finis monotone pour des opérateurs de diffusion fortement anisotropes sur des maillages de triangles non structurés. C.R. Math. 341(12), 787-792 (2005)

31. Potier, C.L.: A nonlinear finite volume scheme satisfying maximum and minimum principles for diffusion operators. Int. J. Finite Vol. 6(2), 1-20 (2009)

32. Sammon, P.H.: An Analysis of Upstream Differencing. SPE Reservoir Engineering 3(03), 1053-1056 (1988). DOI 10.2118/14045-PA

33. Schneider, M.: Nonlinear finite volume schemes for complex flow processes and challenging grids. Dissertation, Universitt Stuttgart, Stuttgart (2019). DOI 10.18419/opus-10416

34. Schneider, M., Agélas, L., Enchéry, G., Flemisch, B.: Convergence of nonlinear finite volume schemes for heterogeneous anisotropic diffusion on general meshes. Journal of Computational Physics 351(Supplement C), $80-107$ (2017). DOI 10.1016/j.jcp.2017.09.003

35. Schneider, M., Flemisch, B., Helmig, R.: Monotone nonlinear finite-volume method for nonisothermal two-phase two-component flow in porous media. International Journal for Numerical Methods in Fluids 84(6), 352-381 (2017)

36. Schneider, M., Flemisch, B., Helmig, R., Terekhov, K., Tchelepi, H.: Monotone nonlinear finite-volume method for challenging grids. Computational Geosciences 22(2), 565-586 (2018). DOI 10.1007/s10596-017-9710-8

37. Schneider, M., Gläser, D., Flemisch, B., Helmig, R.: Nonlinear finite-volume scheme for complex flow processes on corner-point grids. In: C. Cancès, P. Omnes (eds.) Finite Volumes for Complex Applications VIII Hyperbolic, Elliptic and Parabolic Problems, pp. 417-425. Springer International Publishing (2017)

38. Schneider, M., Gläser, D., Flemisch, B., Helmig, R.: Comparison of finite-volume schemes for diffusion problems. Oil Gas Sci. Technol. - Rev. IFP Energies nouvelles 73 (2018). DOI 10.2516/ogst/2018064

39. Terekhov, K., Mallison, B., Tchelepi, H.: Cell-centered nonlinear finite-volume methods for the heterogeneous anisotropic diffusion problem. J. Comput. Phys. 330, 245-267 (2017)

40. Vignal, M.: Convergence of a finite volume scheme for an elliptic-hyperbolic system. ESAIM: Mathematical Modelling and Numerical Analysis 30(7), 841-872 (1996)

41. Yuan, G., Sheng, Z.: Monotone finite volume schemes for diffusion equations on polygonal meshes. J. Comput. Phys. 227(12), 6288-6312 (2008) 